\title{
Site-Selective Acylation of Pyranosides with Oligopeptide Catalysts
}

Alexander Seitz, ${ }^{\mathrm{a}}$ Raffael C. Wende, ${ }^{\mathrm{a}}$ Emily Roesner, ${ }^{\mathrm{a}}$ Dominik Niedek, ${ }^{\mathrm{a}}$ Christopher Topp, ${ }^{\mathrm{a}}$ Avene C. Colgan, ${ }^{\mathrm{b}}$ Eoghan M. McGarrigle, ${ }^{\mathrm{b}}$ and Peter R. Schreiner*a

a Institute of Organic Chemistry, Justus Liebig University, Heinrich-Buff-Ring 17, 35392 Giessen (Germany)

${ }^{b}$ Centre for Synthesis \& Chemical Biology, UCD School of Chemistry, University College Dublin, Belfield, Dublin 4 (Ireland)

*Email:prs@uni-giessen.de

\section{Table of contents}

Determination of yields and selectivity via ${ }^{1} \mathrm{H}-\mathrm{NMR}$

S2-S13

Catalyst screening and DoE

S14-S17

Additional results

$\mathrm{S} 18$

Table of literature known procedures

S19-S21

NMR data

$\mathrm{S} 22-72$

References

S73 
Determination of yields and selectivity via NMR

The ratio of the respective three different acetylated products was determined via NMR. Therefore, NMR spectra of the crude reaction mixtures were recorded, and the ratio of the products as well as the yields could be obtained via integration of the corresponding signals. The signals which have been used are shown for the different substrates.

Acetylation of methyl 4,6-O-benzylidene-a,D-glucopyranoside (3)

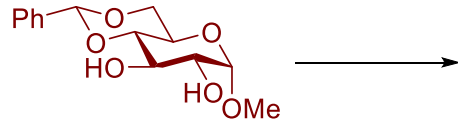

3

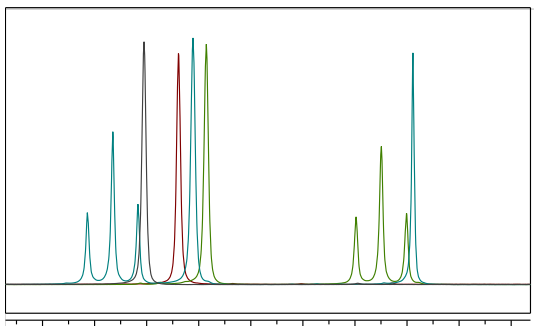

$\begin{array}{lllllllllll}5.65 & 5.60 & 5.55 & 5.50 & 5.45 & 5.40 & 5.35 & 5.30 & 5.25 & 5.20\end{array}$ f1 (ppm)

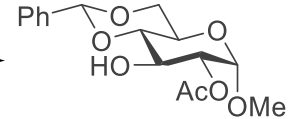

$3 a$

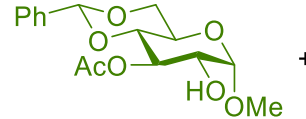

$3 \mathbf{b}$

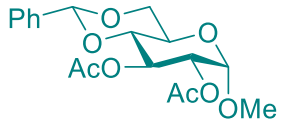

$3 c$

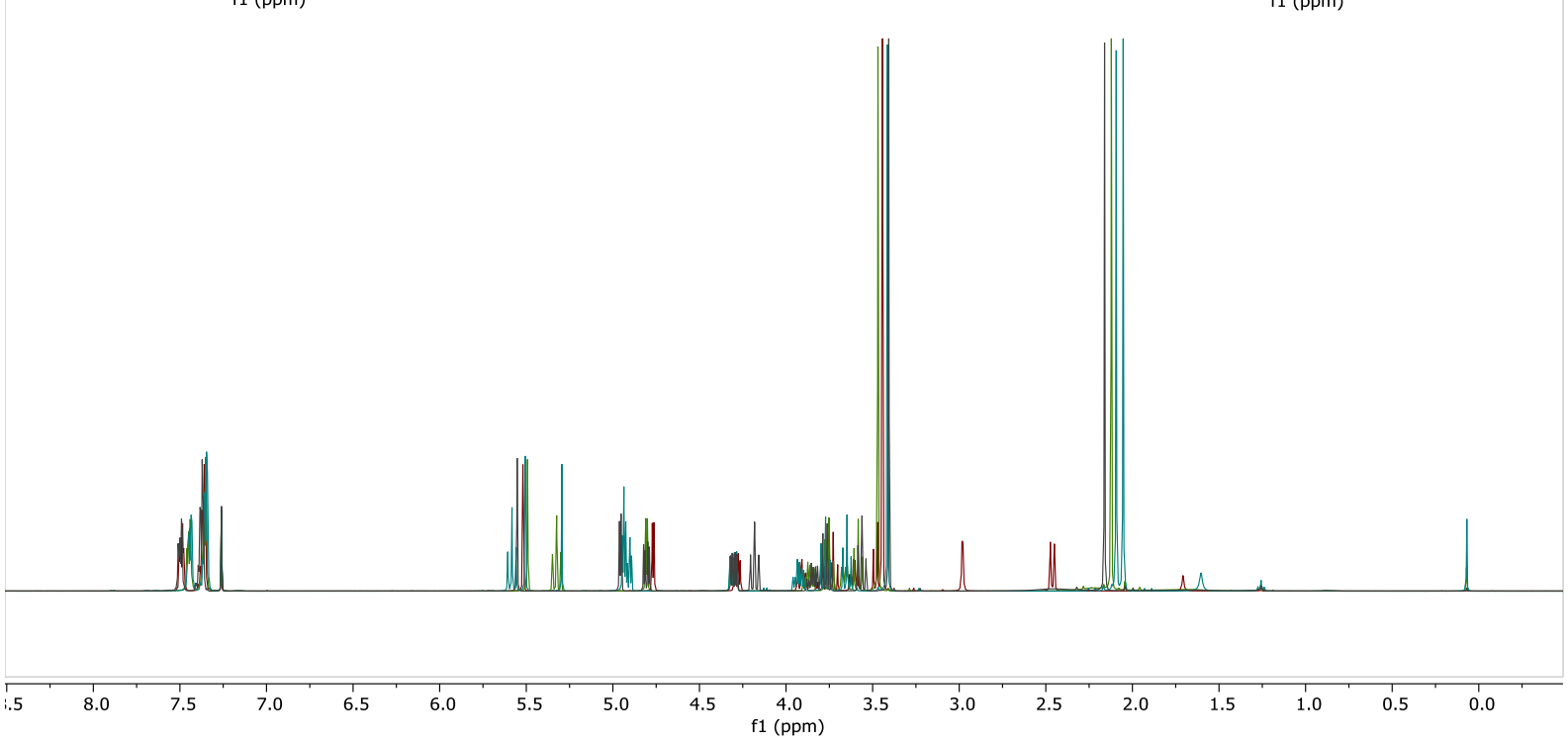

Figure 1 Superimposed ${ }^{1} \mathrm{H}$ NMR spectra of methyl 4,6-O-benzylidene- $\alpha, \mathrm{D}-$ glucopyranoside (3) (red) and the isolated acetylated products $\mathbf{3 a}$ (grey), $\mathbf{3 b}$ (green), and $\mathbf{3 c}$ (turquoise) used for the determination of conversion and selectivity. 
Acetylation of methyl 4,6-O-methylidene- $\alpha, D-$ glucopyranoside (4)
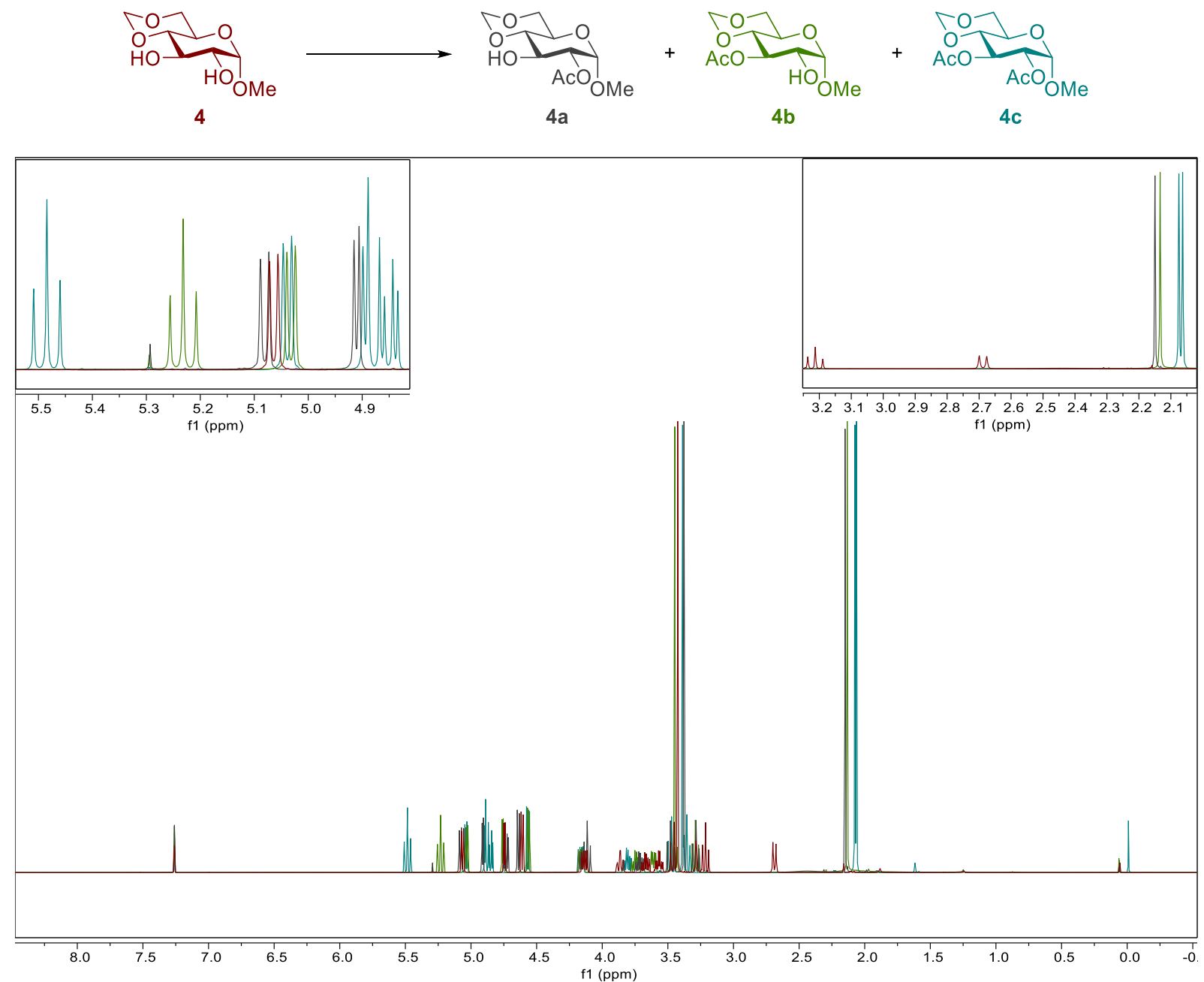

Figure S2 Superimposed ${ }^{1} \mathrm{H}$ NMR spectra of methyl 4,6-O-methylidene-a,D-glucopyranoside (4) (red) and the isolated acetylated products $\mathbf{4 a}$ (grey), $\mathbf{4 b}$ (green), and $\mathbf{4 c}$ (turquoise) used for the determination of conversion and selectivity. 


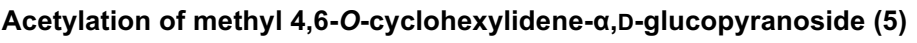

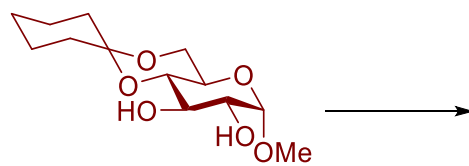

5

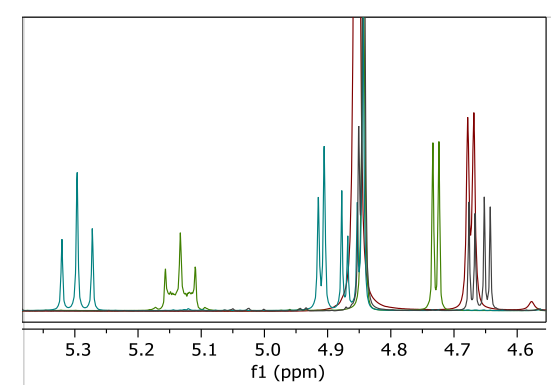

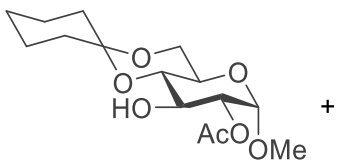

5 a

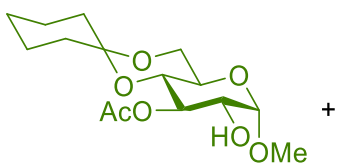

$5 b$

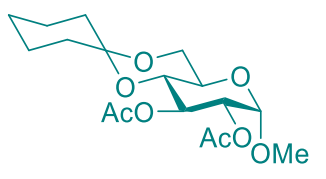

$5 c$
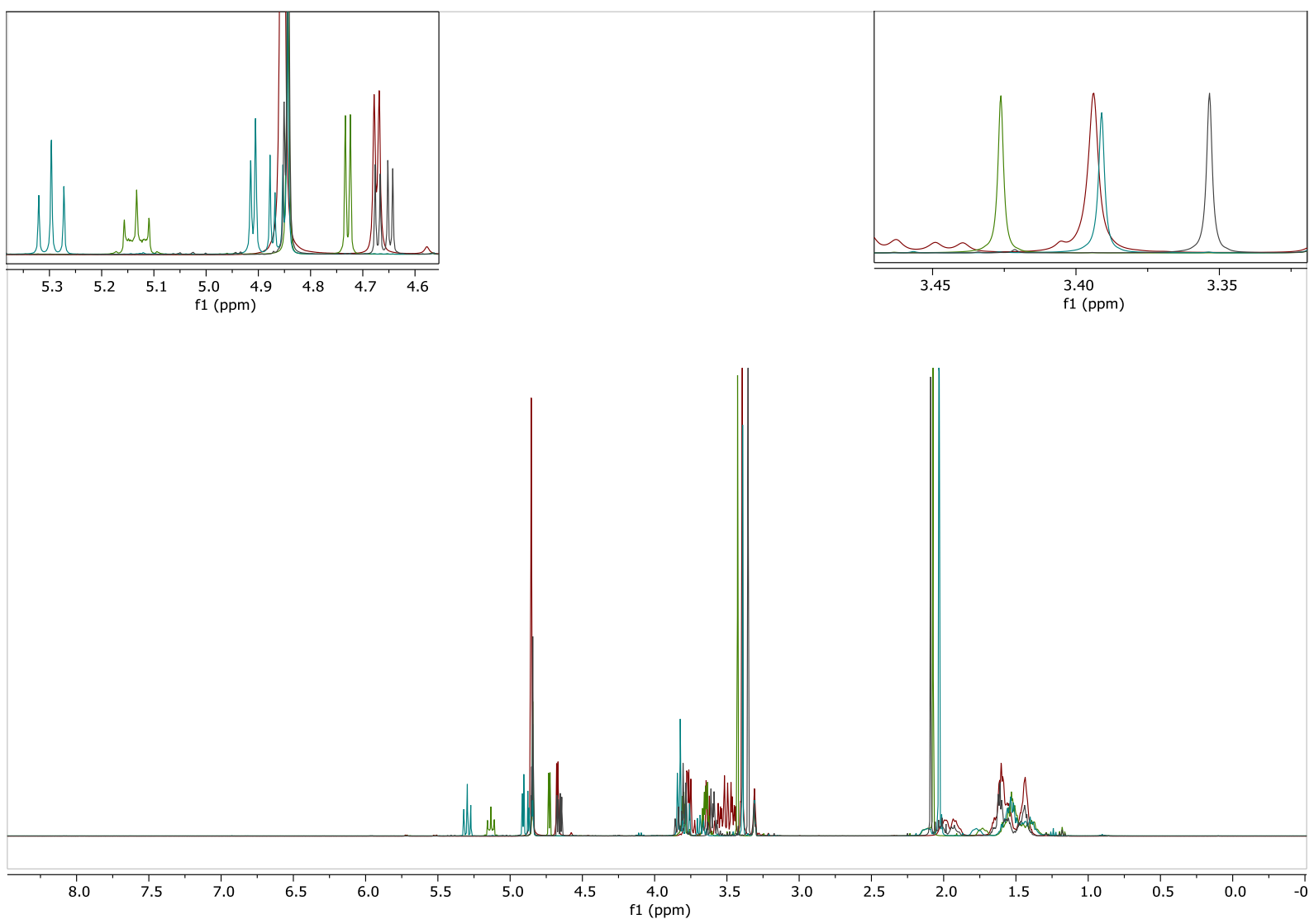

Figure S3 Superimposed ${ }^{1} \mathrm{H}$ NMR spectra of methyl 4,6-O-cyclohexylidene- $\alpha, \mathrm{D}-$ glucopyranoside (5) (red) and the isolated acetylated products $\mathbf{5 a}$ (grey), $\mathbf{5 b}$ (green), and $\mathbf{5 c}$ (turquoise) used for the determination of conversion and selectivity. 
Acetylation of methyl 4,6-O-benzylidene- $\beta$,D-glucopyranoside (6)

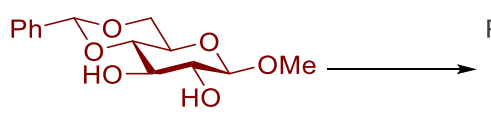

6

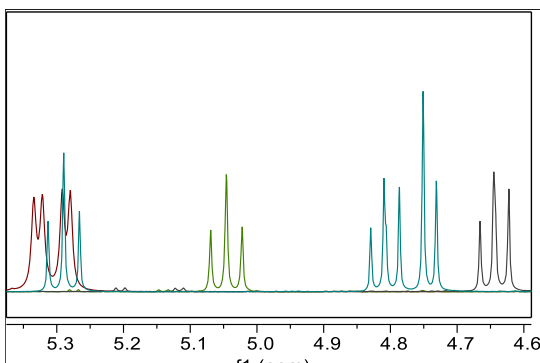

f1 (ppm)

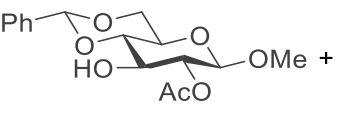

$6 a$

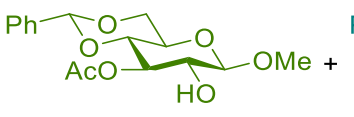

$6 b$

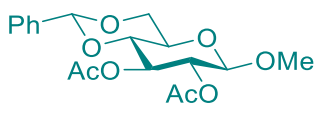

$6 c$

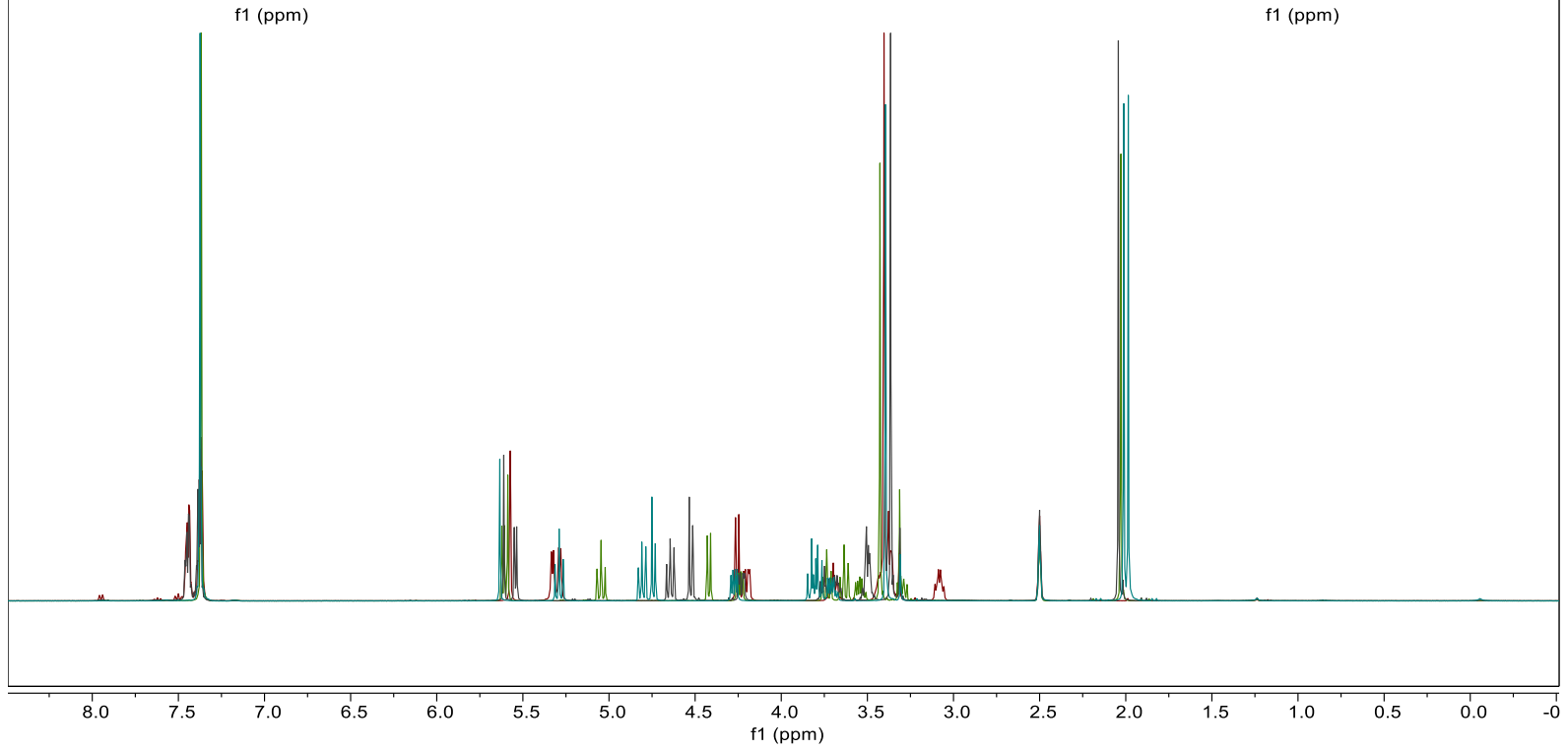

Figure S4 Superimposed ${ }^{1} \mathrm{H}$ NMR spectra of methyl 4,6-O-benzylidene- $\beta, \mathrm{D}$-glucopyranoside (6) (red) and the isolated acetylated products $\mathbf{6 a}$ (grey), 6b (green), and $\mathbf{6 c}$ (turquoise) used for the determination of conversion and selectivity. 


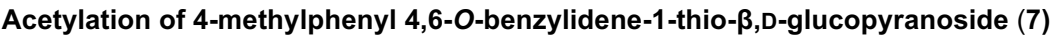
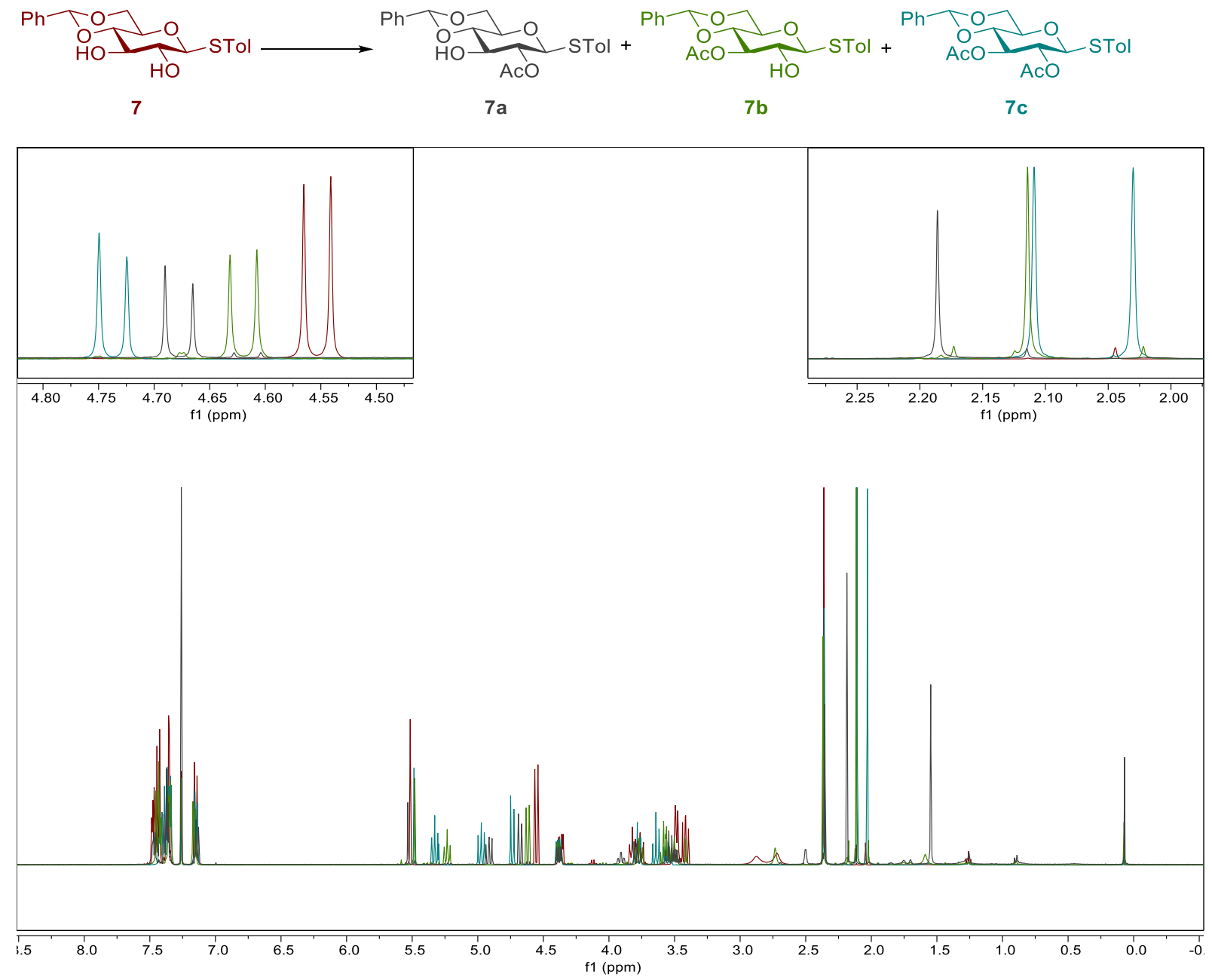

Figure S5 Superimposed ${ }^{1} \mathrm{H}$ NMR spectra of 4-methylphenyl 4,6-O-benzylidene-1-thio- $\beta, \mathrm{D}$-glucopyranoside (7) (red) and the isolated acetylated products $7 \mathbf{a}$ (grey), $\mathbf{7 b}$ (green), and $\mathbf{7 c}$ (turquoise) used for the determination of conversion and selectivity. 
Acylation of methyl 4,6-O-benzylidene-a,D-glucopyranoside (3)

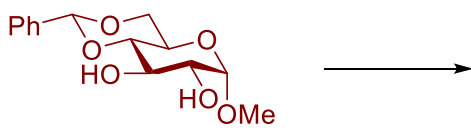

3

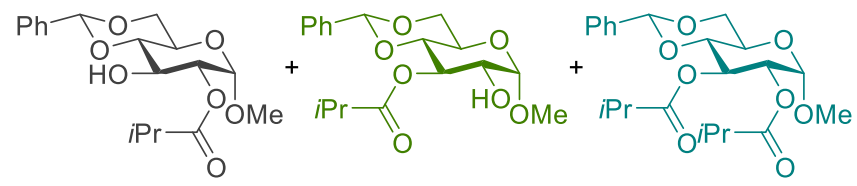

$8 a$

$8 b$

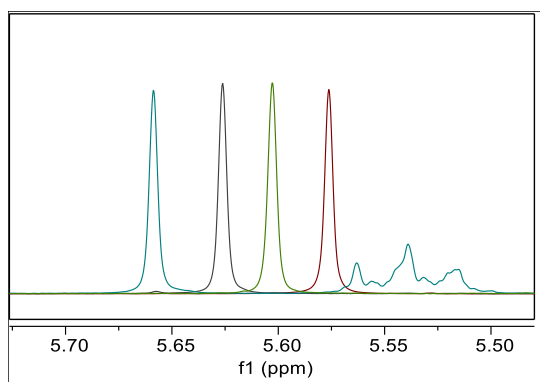

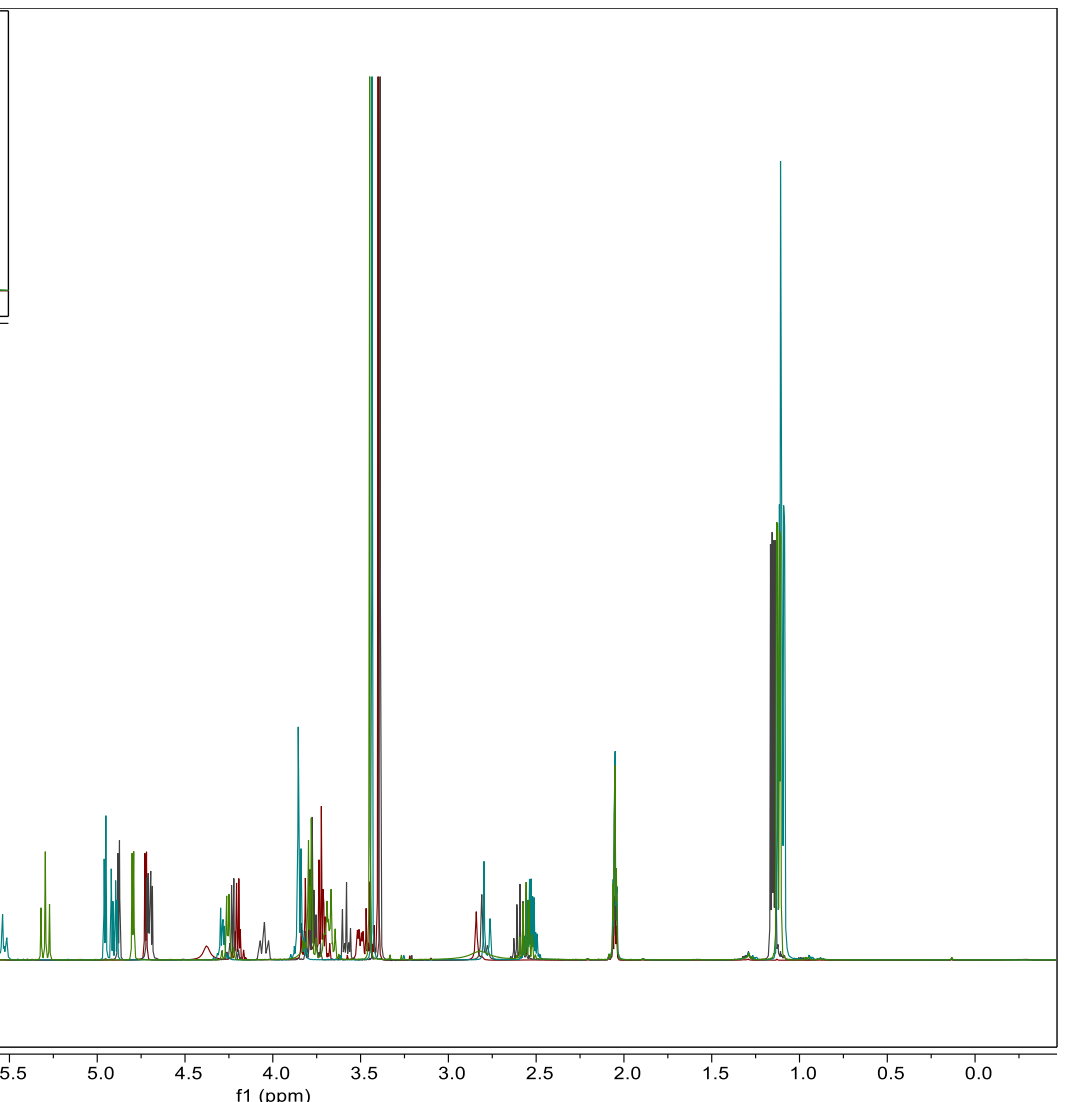

Figure S6 Superimposed ${ }^{1} \mathrm{H}$ NMR spectra of methyl 4,6-O-benzylidene- $\mathrm{S}$,D-glucopyranoside (3) (red) and the isolated acylated products $\mathbf{8 a}$ (grey), $\mathbf{8 b}$ (green), and $\mathbf{8 c}$ (turquoise) used for the determination of conversion and selectivity. 
Acylation of methyl 4,6-O-methylidene- $\alpha$,D-glucopyranoside (4)

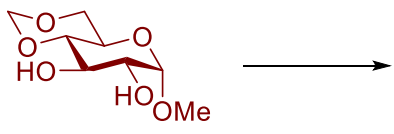

4

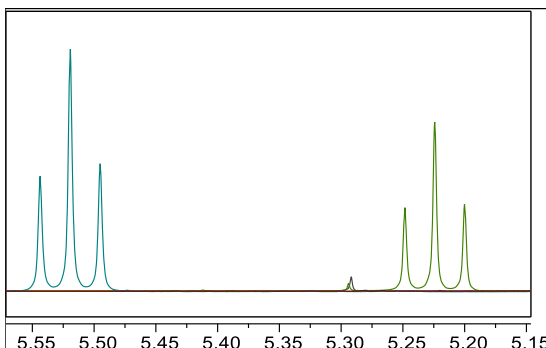

f1 (ppm)

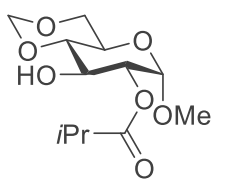

$9 a$

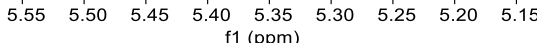

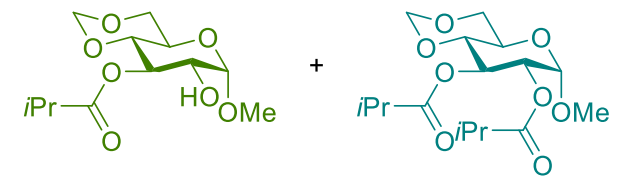

$9 c$

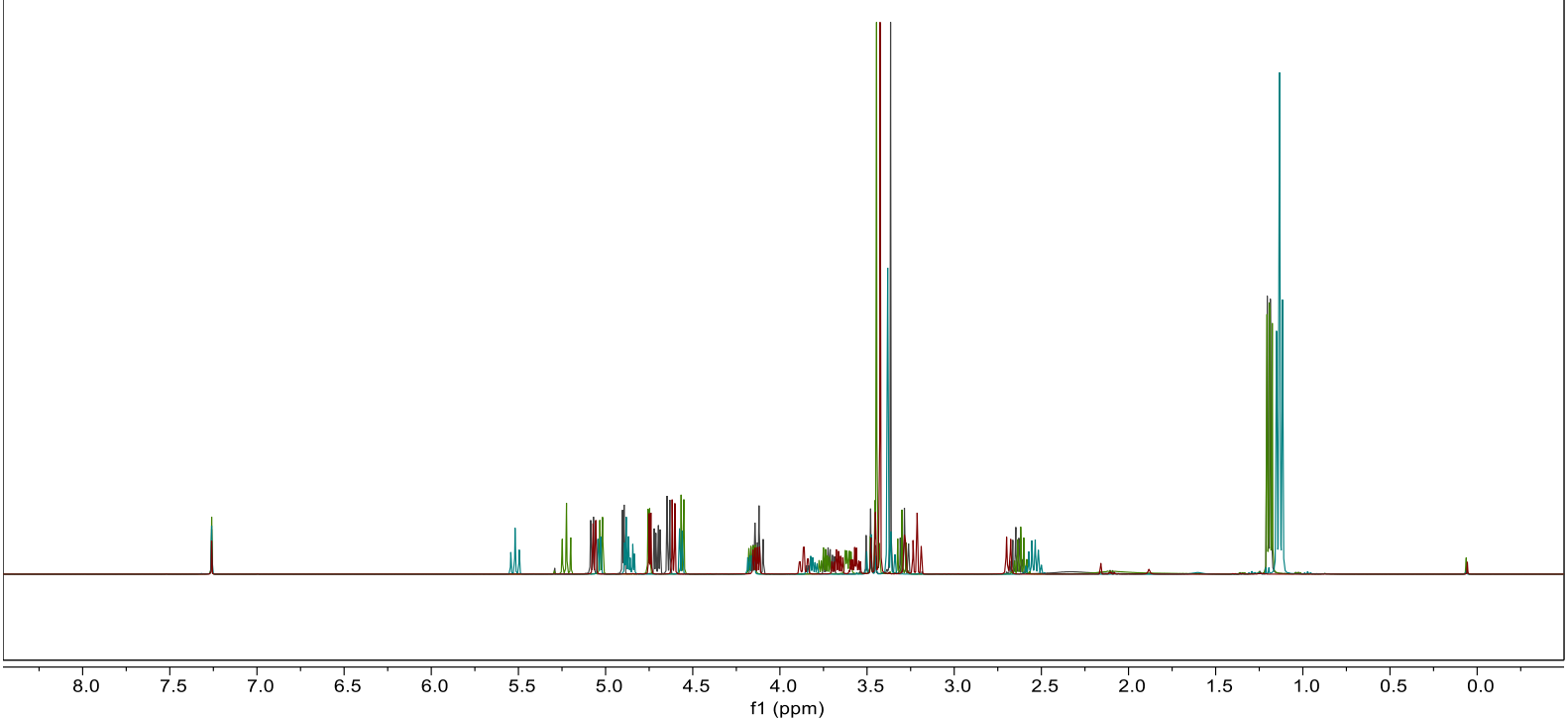

Figure S7 Superimposed ${ }^{1} \mathrm{H}$ NMR spectra of methyl 4,6-O-methylidene- $\alpha, \mathrm{D}-$ glucopyranoside (4) (red) and the isolated acylated products 9a (grey), 9b (green), and 9c (turquoise) used for the determination of conversion and selectivity. 


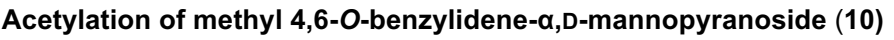

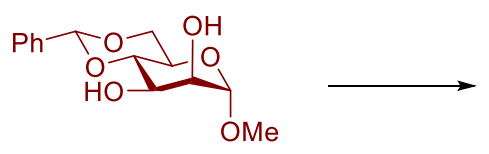

10

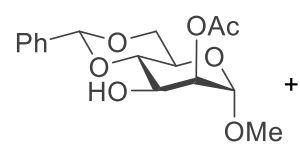

$10 a$

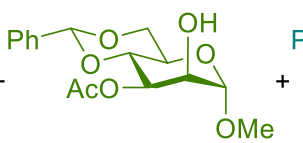

$10 \mathrm{~b}$

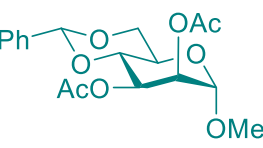

$10 \mathrm{c}$

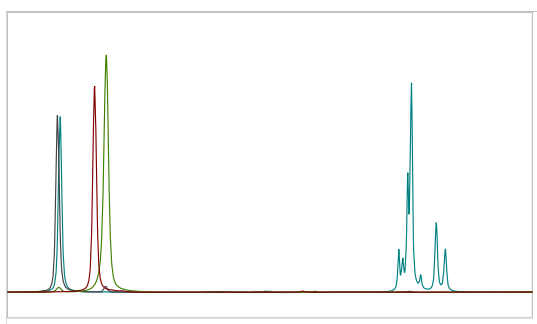

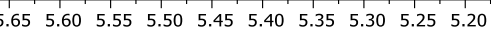
f1 (ppm)

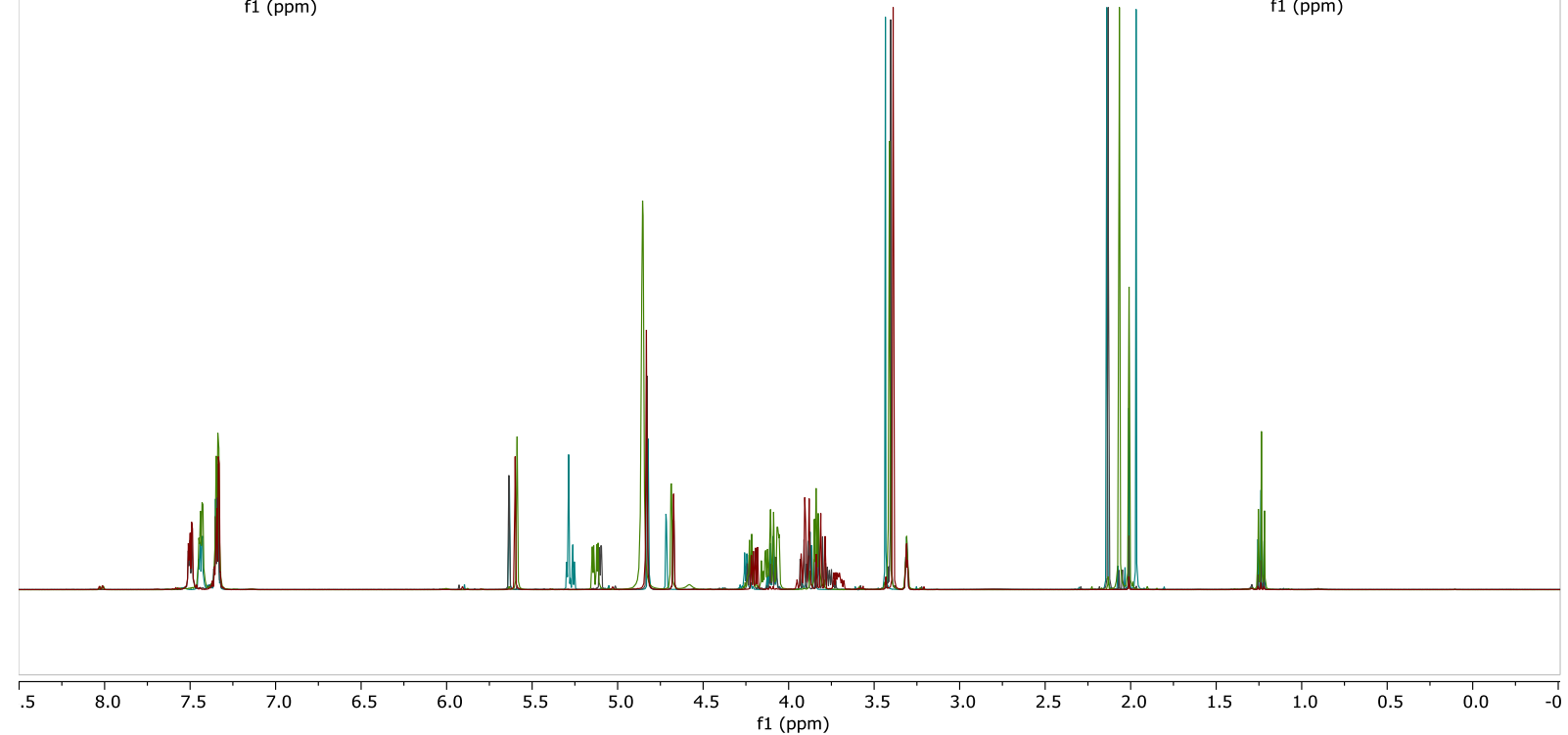

Figure S8 Superimposed ${ }^{1} \mathrm{H}$ NMR spectra of methyl 4,6-O-benzylidene- $\alpha, \mathrm{D}-\mathrm{mannopyranoside} \mathrm{(10)} \mathrm{(red)} \mathrm{and} \mathrm{the} \mathrm{isolated}$ acetylated products 10a (grey), 10b (green), and 10c (turquoise) used for the determination of conversion and selectivity. 


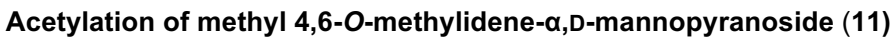

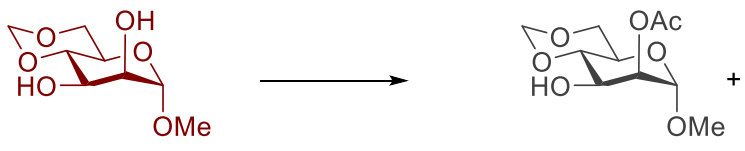

11

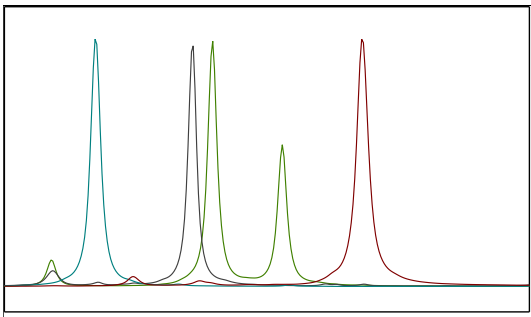

$\begin{array}{lllllllll}3.42 & 3.41 & 3.40 & 3.39 & 3.38 & 3.37 & 3.36 & 3.35 & 3.34\end{array}$

$1(\mathrm{ppm})$

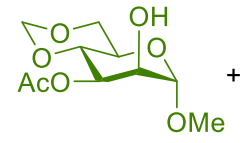

$11 b$

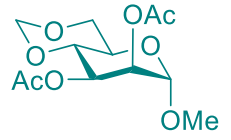

$11 \mathrm{c}$

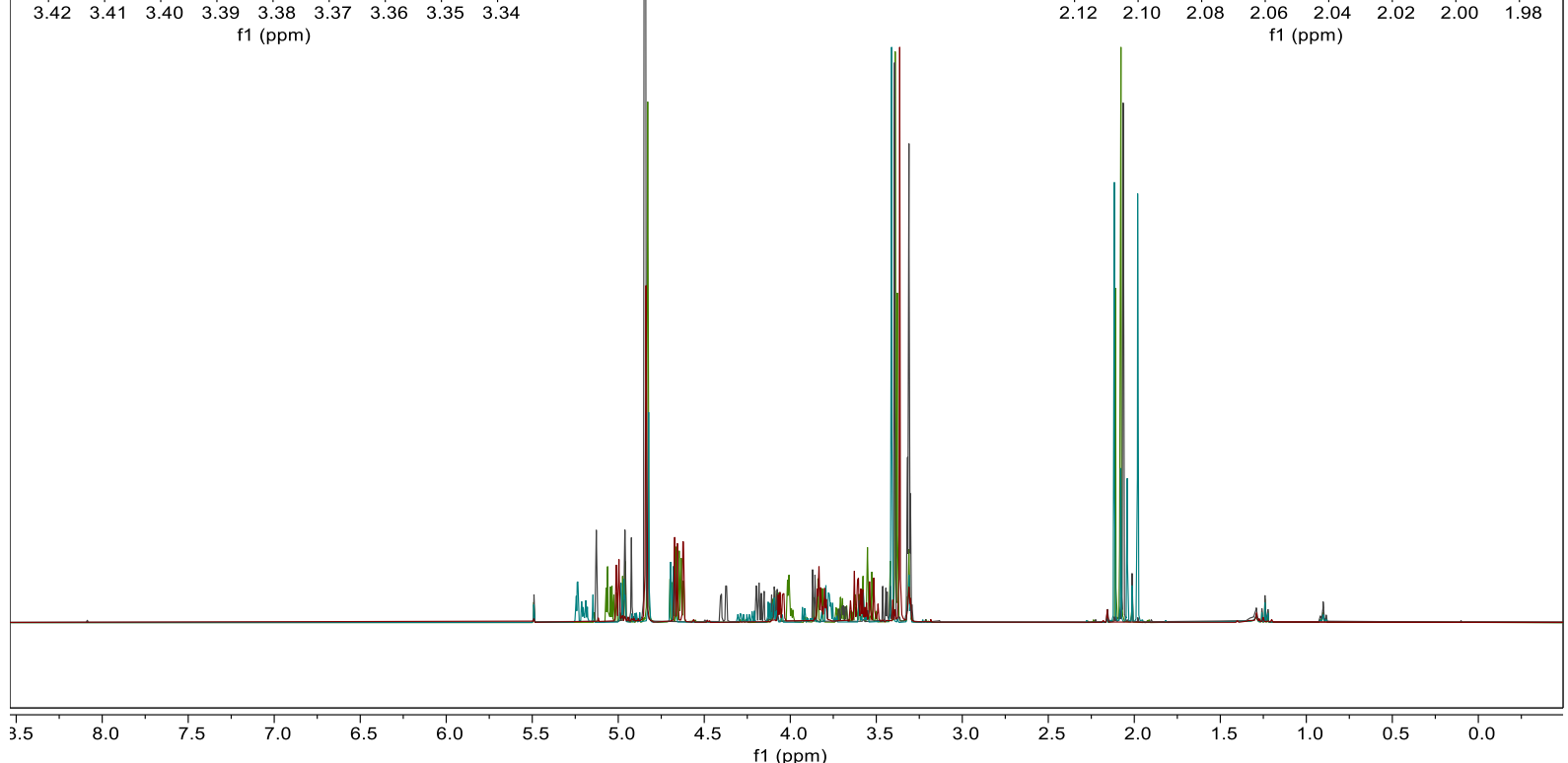

Figure S9 Superimposed ${ }^{1} \mathrm{H}$ NMR spectra of methyl 4,6-O-methylidene- $\alpha, \mathrm{D}-$ mannopyranoside (11) (red) and the isolated acetylated products 11a (grey), 11 (green), and 11c (turquoise) used for the determination of conversion and selectivity. 


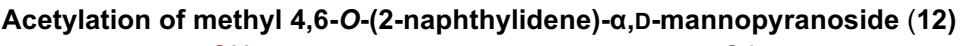
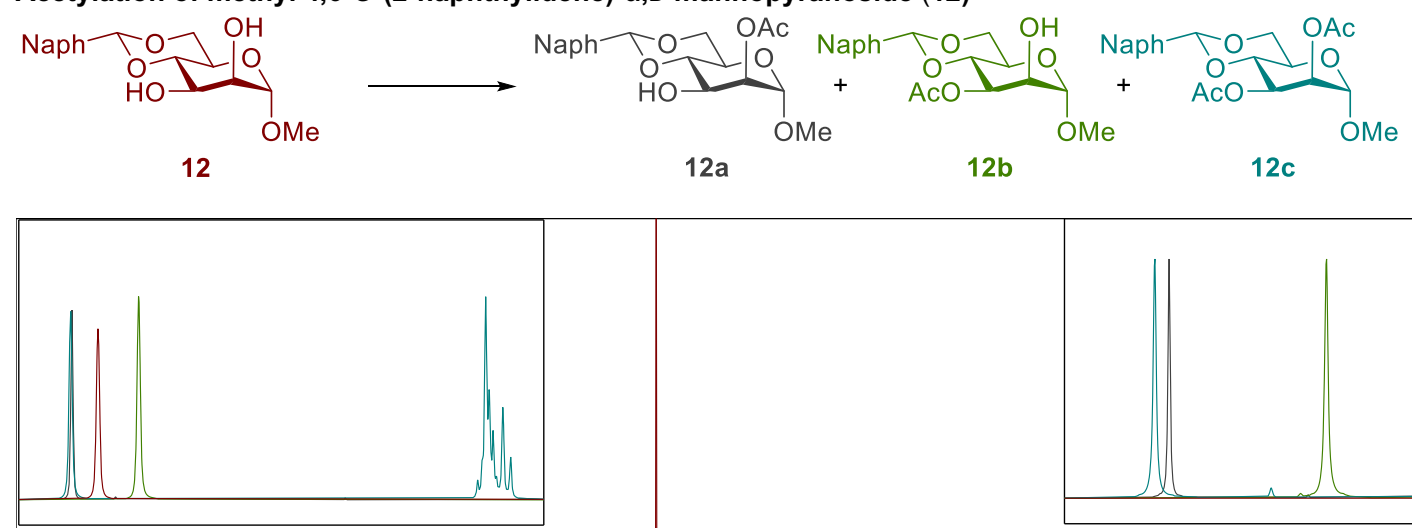

$12 b$

$12 \mathrm{c}$

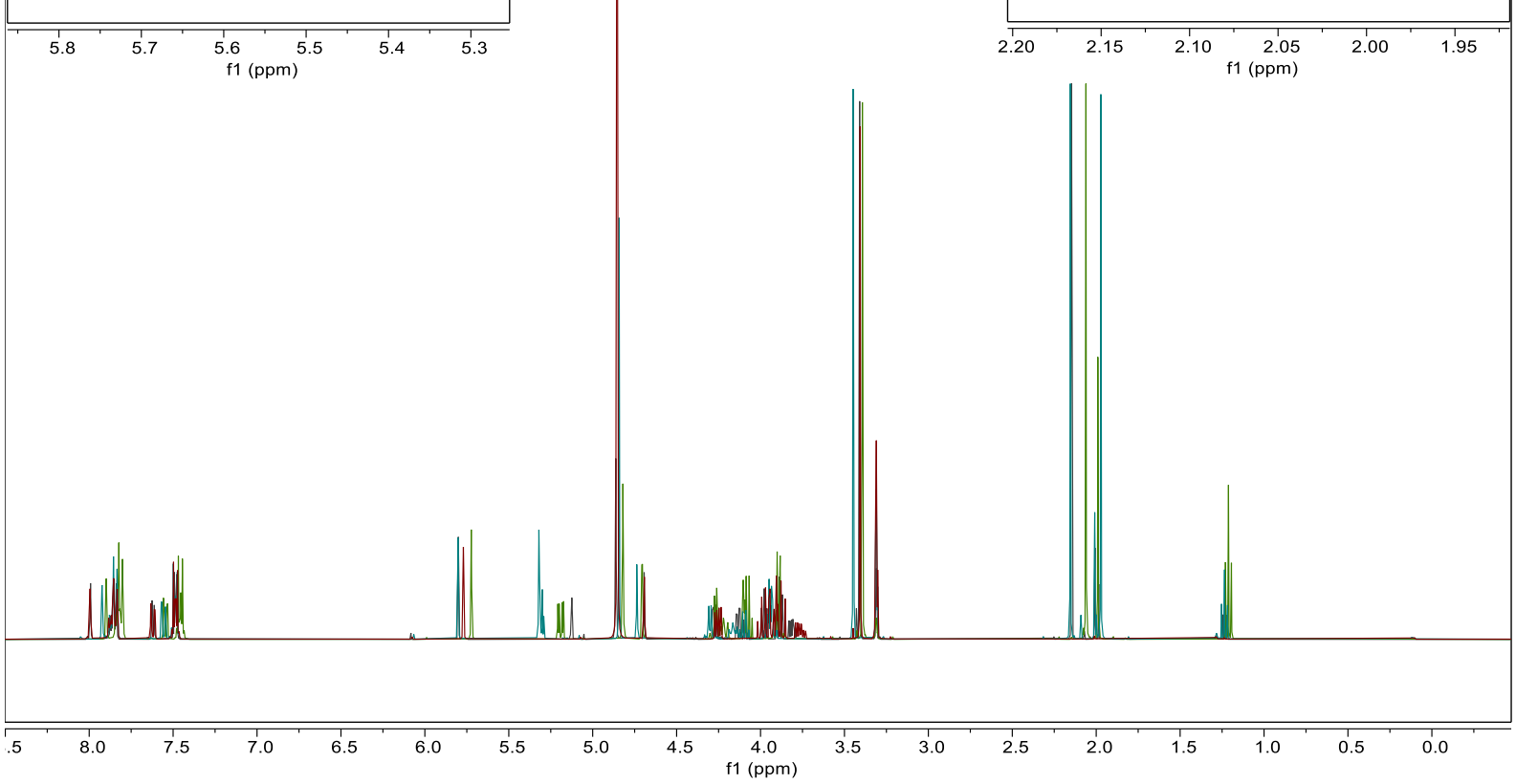

Figure S10 Superimposed ${ }^{1} \mathrm{H}$ NMR spectra of methyl 4,6-O-(2-naphtylidene)-a,D-mannopyranoside (12) (red) and the isolated acetylated products 12a (grey), 12 (green), and 12c (turquoise) used for the determination of conversion and selectivity. 
Acylation of methyl 4,6-O-benzylidene-a,D-mannopyranoside (10) with isobutyric anhydride
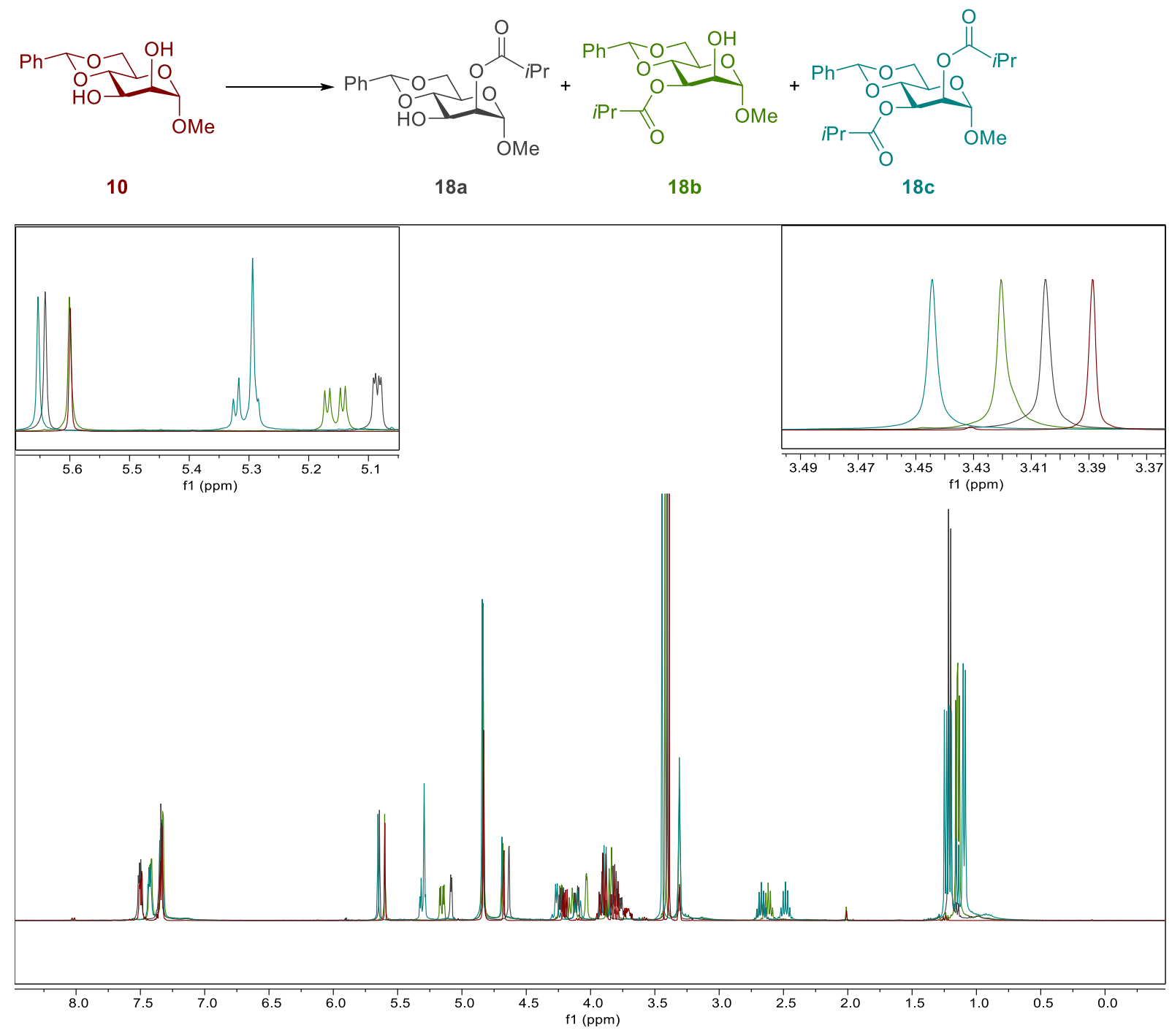

Figure S11 Superimposed ${ }^{1} \mathrm{H}$ NMR spectra of methyl 4,6-O-benzylidene- $\alpha$,D-mannopyranoside (10) (red) and the isolated acylated products 18a (grey), 18b (green), and 18c (turquoise) used for the determination of conversion and selectivity. 
Acetylation of methyl 4,6-O-benzylidene-a,D-galactopyranoside (13)

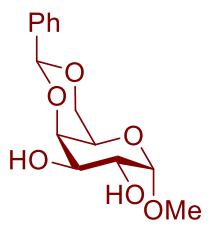

13

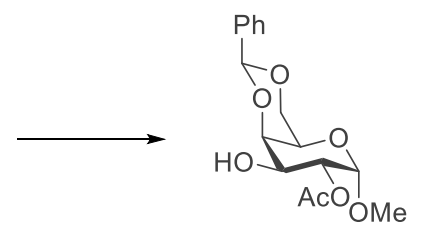

$13 a$

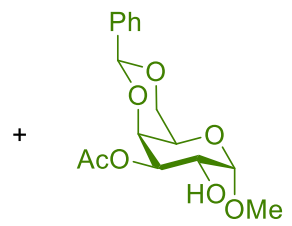

$13 b$

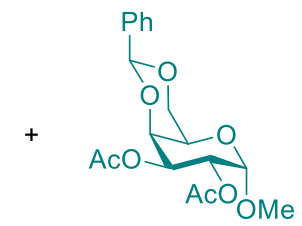

$13 c$

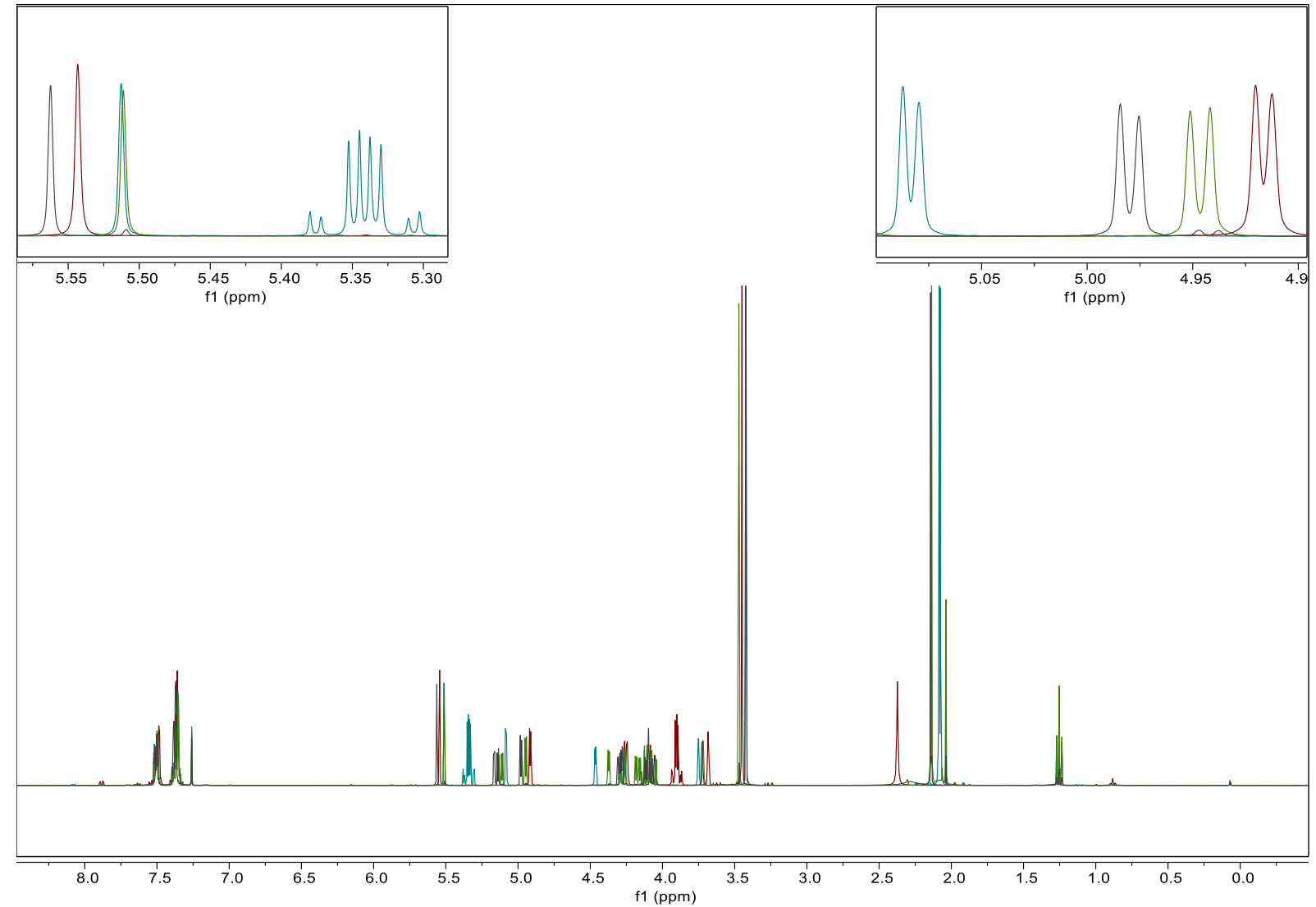

Figure S12 Superimposed ${ }^{1} \mathrm{H}$ NMR spectra of methyl 4,6-O-benzylidene- $\alpha, \mathrm{D}-$ galactopyranoside (13) (red) and the isolated acetylated products 13a (grey), 13b (green), and 13c (turquoise) used for the determination of conversion and selectivity. 


\section{Optimization of reaction conditions}

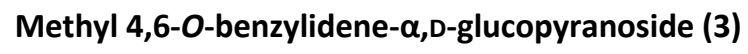

\section{Catalyst screening}

Table S1 Acetylation of methyl 4,6-O-benzylidene- $\alpha$-D-glucopyranoside (3) with different oligopeptide catalysts.

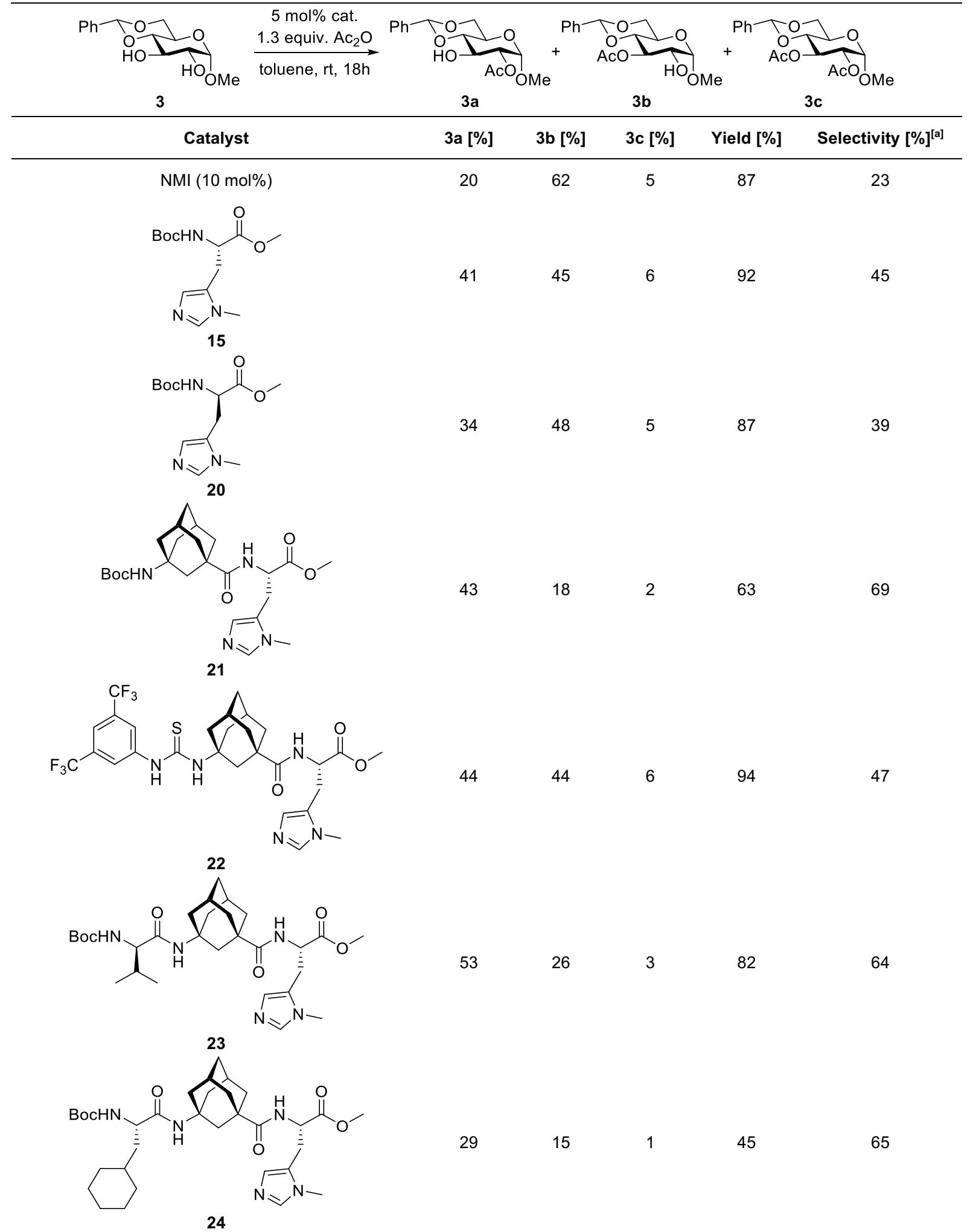


<smiles>COC(=O)N[C@@H](Cc1c[nH]c2ccccc12)C(=O)NC12CC3CC(C1)CC(C(=O)N[C@H](Cc1cncn1C)C(=O)OC(C)(C)C)(C3)C2</smiles><smiles>COC(=O)[C@H](Cc1cncn1C)NC(=O)C12CC3CC1CC(NC(=O)[C@H](Cc1ccccc1)NC(=O)OC(C)(C)C)(C3)C2</smiles><smiles>COC(=O)C12CC3CC(CC(NC(=O)[C@H](Cc4cncn4C)NC(=O)OCc4ccccc4)(C3)C1)C2</smiles><smiles>COC(=O)N[C@@H](Cc1cncn1C)C(=O)NC12CC3CC(C1)CC(C(=O)OC)(C3)C2</smiles>

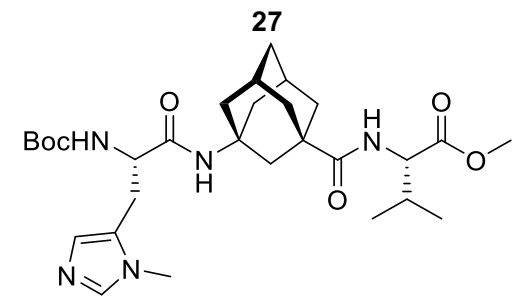<smiles>COC(=O)[C@H](NC(=O)C12CC3CC(NC(=O)[C@H](Cc4cncn4C)NC(=O)OC(C)(C)C)(CC3[C@H]1C)C2)C(C)C</smiles><smiles></smiles>

20

42

64

52

69

44

55

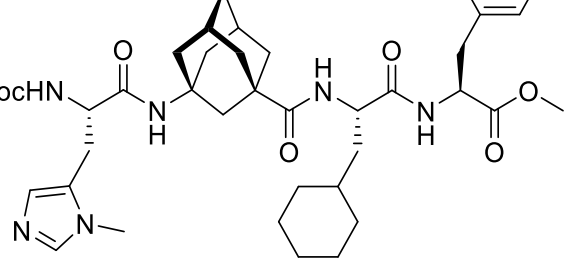

24

54

61

44

64

54

69

$21 \quad 10 \quad>95$

61

55 
<smiles>COC(=O)c1ccccc1C[C@H](NC(=O)[C@H](CC1CCCCC1)NC(=O)C12CC3CC(CC(NC(=O)[C@@H](Cc4ccccc4)Cc4cncn4C)(C3)C1)C2)C(=O)O</smiles>

31

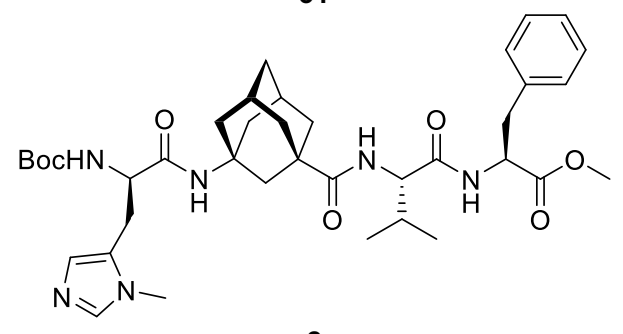

70 $=$

32<smiles>COC(=O)c1ccccc1C[C@H](NC(=O)[C@H](Cc1ccccc1)NC(=O)[C@H](Cc1ccccc1)NC(=O)C12CC3CC(CC(NC(=O)c4ccccc4)(C3)C1)C2)C(C)C</smiles>

33<smiles>COC(=O)[C@H](Cc1ccccc1)NC(=O)C12CC3CC(CC(NC(=O)C45CC6CC(CC(NC(=O)[C@H](Cc7cncn7C)NC(=O)OC(C)(C)C)(C6)C4)C5)(C3)C1)C2</smiles>

\section{Design of Experiments}

To optimize the reaction conditions, a Custom Design was carried out using JMP ${ }^{\circledR}{ }^{1}$

The desired responses were optimized as follows:

Yield 3c

Yield 3a

Yield $\mathbf{3 b}$

Extant 3

$$
\begin{aligned}
& \text { minimize } \\
& \text { maximize } \\
& \text { minimize }
\end{aligned}
$$

minimize lower limit $=0$

upper limit $=100$

lower limit $=0$

lower limit $=0$

The investigated variables and their range were set to the following values: Concentration of the reaction continuous variable 


$\begin{array}{lll}\text { Catalyst Loading } & \text { continuous variable } & 0.1 \text { to } 10 \mathrm{~mol} \% \\ \text { Amount acetic anhydride } & \text { continuous variable } & 1.0 \text { to } 10 \text { equiv. } \\ \text { Reaction time } & \text { continuous variable } & 1 \text { to } 18 \mathrm{~h} \\ \text { Temperature } & \text { continuous variable } & -20 \text { to } 20^{\circ} \mathrm{C}\end{array}$

The optimized reaction conditions are used to improve the outcome of the reaction (see Table 1, Entry 5).

Table S2 Reactions performed for the acetylation of methyl 4,6-O-benzylidene- $\alpha$-D-glucopyranoside (3) given by JMP ${ }^{\circledR}$ for the Custom Design.

\begin{tabular}{|c|c|c|c|c|c|c|c|c|c|}
\hline \multirow[b]{2}{*}{ Entry } & 3 & $\mathrm{O}_{\mathrm{OMe}}$ & \multirow{2}{*}{$\begin{array}{c}\begin{array}{c}\text { catalyst } 2 \\
\mathrm{Ac}_{2} \mathrm{O}\end{array} \\
\text { toluene } \\
\\
\mathrm{Ac}_{2} \mathrm{O} \\
\text { equiv. }\end{array}$} & \multirow{2}{*}{$\begin{array}{r} \\
\\
\text { rt } \\
{[\mathrm{Ph}]}\end{array}$} & \multirow{2}{*}{$\frac{{ }^{t} \mathrm{O} O}{\left.{ }^{\circ} \mathrm{C}\right]}$} & \multirow{2}{*}{\multicolumn{2}{|c|}{$3 a[\%] \quad 3 b[\%]$}} & \multicolumn{2}{|c|}{ 3c } \\
\hline & $\begin{array}{l}\text { conc. } \\
\text { [mol/L) }\end{array}$ & $\begin{array}{c}\text { cat. } \\
\text { [mol\%] }\end{array}$ & & & & & & $3 c[\%]$ & $\begin{array}{c}\text { Starting material } \\
{[\%]}\end{array}$ \\
\hline 1 & 0.005 & 10 & 10 & 1 & 20 & 69 & 19 & 12 & 0 \\
\hline 2 & 0.0525 & 5.05 & 5.5 & 18 & 0 & 0 & 11 & 89 & 0 \\
\hline 3 & 0.005 & 10 & 1 & 18 & 20 & 74 & 18 & 3 & 5 \\
\hline 4 & 0.005 & 0.1 & 10 & 18 & 20 & 62 & 19 & 3 & 16 \\
\hline 5 & 0.0525 & 5.05 & 1 & 9.5 & 0 & 57 & 19 & 10 & 14 \\
\hline 6 & 0.005 & 10 & 10 & 18 & -20 & 46 & 15 & 39 & 0 \\
\hline 7 & 0.1 & 10 & 1 & 1 & 20 & 57 & 26 & 8 & 9 \\
\hline 8 & 0.1 & 0.1 & 1 & 1 & -20 & 3 & 2 & 0 & 95 \\
\hline 9 & 0.1 & 0.1 & 1 & 18 & 20 & 46 & 27 & 8 & 21 \\
\hline 10 & 0.1 & 10 & 10 & 18 & 20 & 0 & 0 & 100 & 0 \\
\hline 11 & 0.0525 & 5.05 & 1 & 9.5 & 0 & 58 & 24 & 15 & 2 \\
\hline 12 & 0.005 & 5.05 & 10 & 9.5 & -20 & 70 & 1 & 14 & 0 \\
\hline 13 & 0.0525 & 0.1 & 5.5 & 9.5 & 20 & 56 & 27 & 16 & 1 \\
\hline 14 & 0.0525 & 10 & 5.5 & 9.5 & 0 & 0 & 10 & 90 & 0 \\
\hline 15 & 0.1 & 5.05 & 10 & 9.5 & 20 & 0 & 3 & 97 & 0 \\
\hline 16 & 0.005 & 0.1 & 5.5 & 9.5 & 0 & 26 & 5 & 0 & 69 \\
\hline 17 & 0.1 & 0.1 & 10 & 1 & 0 & 39 & 28 & 17 & 17 \\
\hline 18 & 0.0525 & 5.05 & 5.5 & 1 & 20 & 28 & 27 & 45 & 0 \\
\hline 19 & 0.1 & 5.05 & 5.5 & 9.5 & -20 & 21 & 18 & 58 & 2 \\
\hline 20 & 0.005 & 5.05 & 5.5 & 1 & 0 & 68 & 19 & 5 & 7 \\
\hline 21 & 0.005 & 10 & 1 & 1 & -20 & 19 & 4 & 0 & 77 \\
\hline 22 & 0.1 & 10 & 10 & 1 & -20 & 52 & 19 & 19 & 11 \\
\hline 23 & 0.0525 & 0.1 & 10 & 1 & -20 & 6 & 3 & 1 & 90 \\
\hline 24 & 0.1 & 10 & 1 & 18 & -20 & 49 & 16 & 18 & 20 \\
\hline 25 & 0.005 & 0.1 & 1 & 1 & 20 & 2 & 1 & 0 & 97 \\
\hline 26 & 0.1 & 0.1 & 10 & 18 & -20 & 33 & 17 & 8 & 42 \\
\hline 27 & 0.005 & 0.1 & 1 & 18 & -20 & 9 & 1 & 0 & 89 \\
\hline 28 & 0.005 & 10 & 1 & 18 & 20 & 73 & 17 & 3 & 7 \\
\hline 29 & 0.005 & 0.1 & 10 & 18 & 20 & 64 & 20 & 5 & 10 \\
\hline 30 & 0.0525 & 5.05 & 1 & 9.5 & 0 & 55 & 20 & 16 & 10 \\
\hline 31 & 0.1 & 10 & 10 & 1 & -20 & 41 & 21 & 35 & 3 \\
\hline 32 & 0.005 & 0.1 & 1 & 1 & 20 & 4 & 1 & 0 & 95 \\
\hline 33 & 0.005 & 5.0 & 1.3 & 18 & 0 & 81 & 17 & 2 & 0 \\
\hline
\end{tabular}




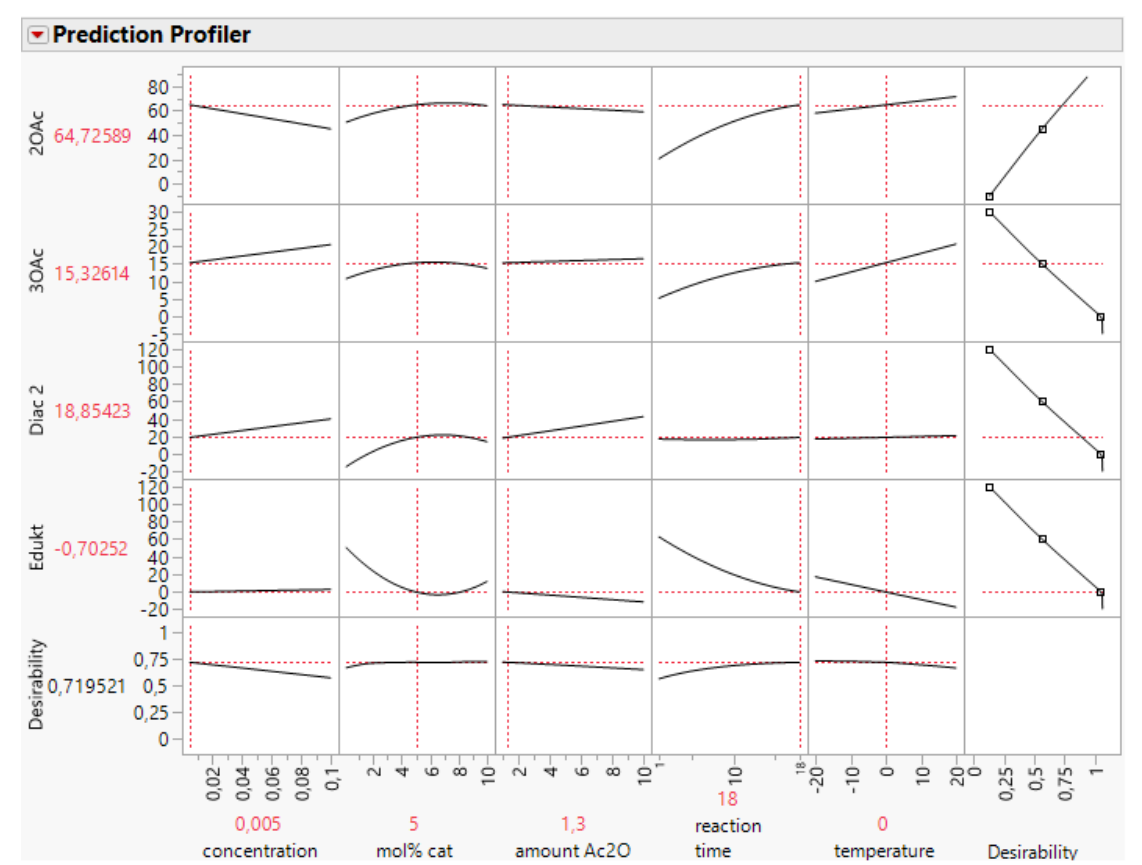

Figure S13 Prediction Profiler for the acetylation of methyl 4,6-O-benzylidene- $\alpha$-D-glucopyranoside (3) generated by JMP ${ }^{\circledR}$.

The Prediction Profiler (Figure S13) is generated via Costume Design using JMP ${ }^{\circledR}$. It is showing how the variables in the model affect the formation of the different products and the consumption of starting material as well as the desirability of the variables on the outcome of the reaction. It can be used to predict the outcome of the reaction for different conditions and was used to find the best conditions.

\section{Methyl 4,6-O-benzylidene-a,D-mannopyranoside (10)}

\section{Design of Experiments}

To optimize the reaction conditions, a Custom Design was carried out using JMP ${ }^{\circledR}{ }^{1}$

The desired responses were optimized as follows:

$\begin{array}{lll}\text { Yield 10c } & \text { minimize } & \text { lower limit }=0 \\ \text { Yield 10a } & \text { maximize } & \text { upper limit }=100 \\ \text { Yield 10b } & \text { minimize } & \text { lower limit }=0 \\ \text { Extant 10 } & \text { minimize } & \text { lower limit }=0\end{array}$

The investigated factors and their range were set to the following values:

$\begin{array}{ll}\text { Concentration of the reaction } & 0.1 \text { to } 0.005 \mathrm{~mol} \cdot \mathrm{L}^{-1} \\ \text { Catalyst Loading } & 0.1 \text { to } 10 \mathrm{~mol} \% \\ \text { Amount acetic anhydride } & 1.0 \text { to } 5.0 \text { equiv. } \\ \text { Reaction time } & 2 \text { to } 30 \mathrm{~h} \\ \text { Temperature } & -30 \text { to } 20^{\circ} \mathrm{C}\end{array}$

The optimized reaction conditions are used to improve the outcome of the reaction (see Table 4, Entry 5). 


\section{Additional Results}

Acylation of methyl 4,6-O-benzylidene-a,D-mannopyranoside (10)

Table S3 Acylation of methyl 4,6-O-benzylidene- ,D-mannopyranoside (10) using isobutyric anhydride.

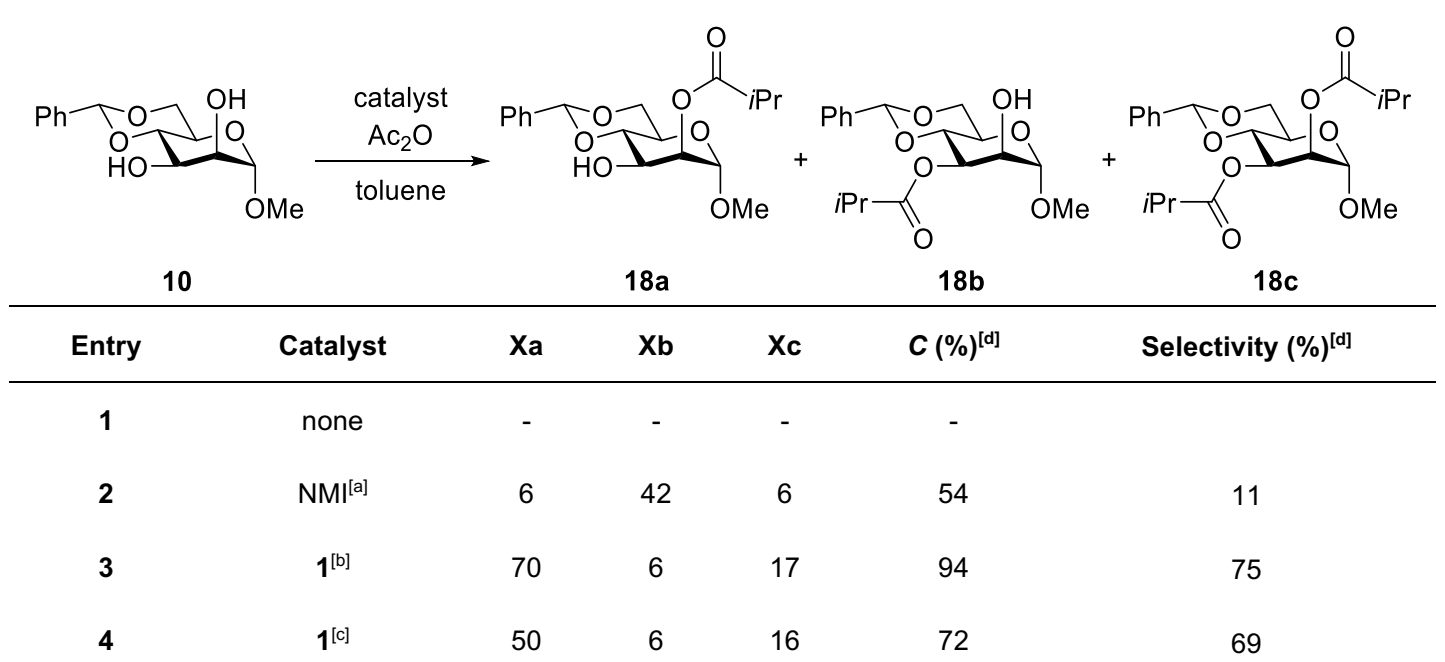

[a] 10 mol\% cat., 1.3 equiv. $\mathrm{Ac}_{2} \mathrm{O}, 18 \mathrm{~h}, \mathrm{rt}, \mathrm{c}=0.01 \mathrm{~mol} \mathrm{~L}^{-1},{ }^{[\mathrm{b}]} 5 \mathrm{~mol} \%$ cat., 1.3 equiv. $\mathrm{Ac}_{2} \mathrm{O}, 18 \mathrm{~h}, \mathrm{rt}, \mathrm{c}=0.01 \mathrm{~mol} \mathrm{L^{-1 }},{ }^{[c]} \mathrm{Conditions}$ obtained by DoE: 10 mol\% cat., 1.0 equiv. $\mathrm{Ac}_{2} \mathrm{O}, 2 \mathrm{~h}, 2{ }^{\circ} \mathrm{C}, 0.005 \mathrm{~mol} \mathrm{~L}^{-1} ;{ }^{[\mathrm{d}]} \mathrm{x}=\mathbf{1 8 a} /(\mathbf{1 8 a}+18 \mathrm{~b}+18 \mathrm{c}) \times 100$. 


\section{Literature known acetylations}

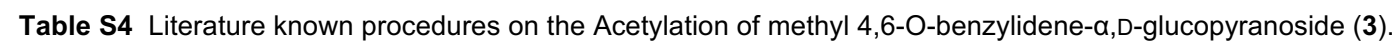

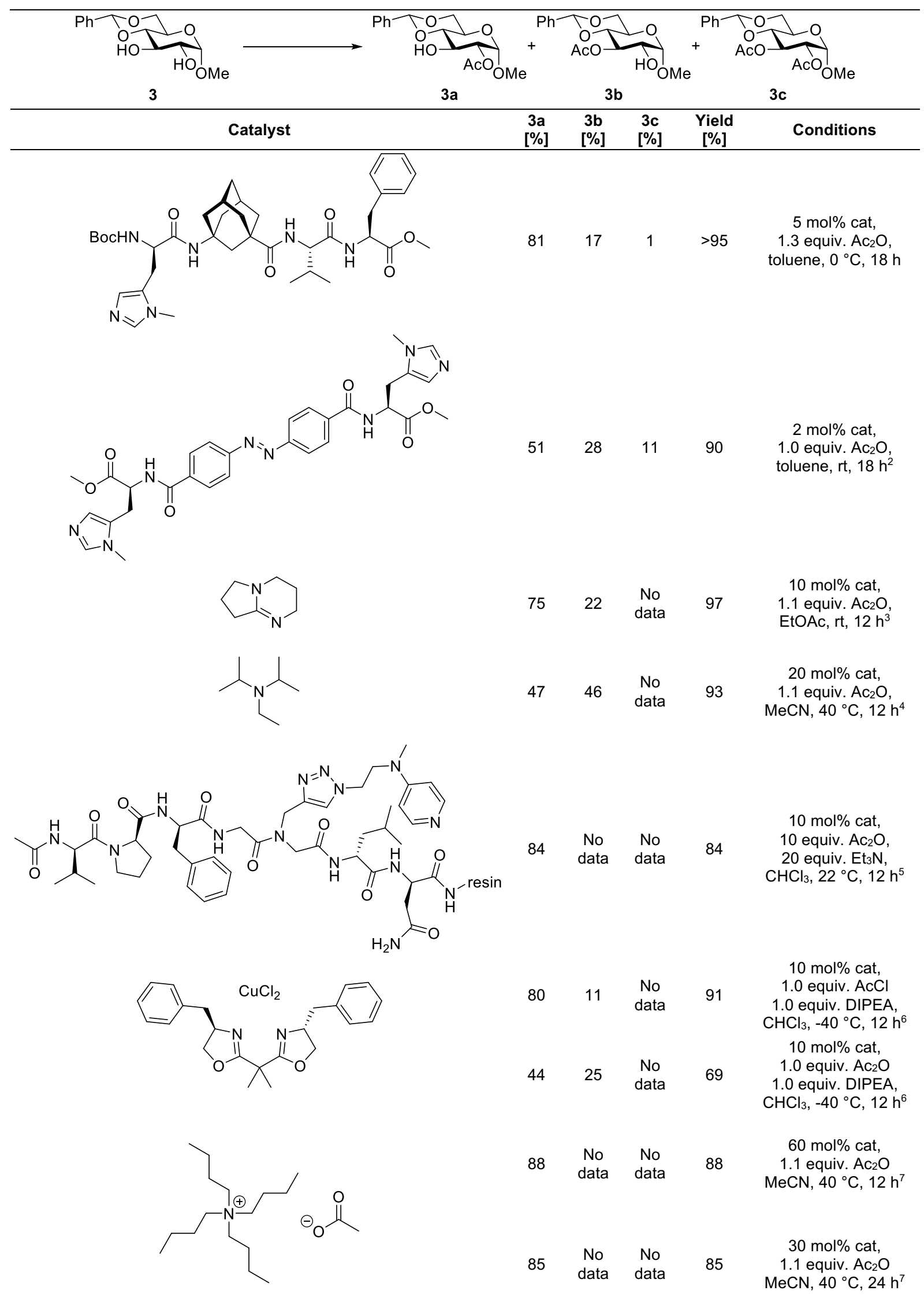




\begin{tabular}{|c|c|c|c|c|c|}
\hline $\mathrm{CuCl}_{2}$ & 15 & 80 & 18 & 100 & $\begin{array}{l}1.0 \text { equiv. metal salt } \\
2.0 \text { equiv. } \mathrm{Ac}_{2} \mathrm{O} \\
2.0 \text { equiv. } \mathrm{NaH} \\
\mathrm{DMG}, \mathrm{rt}, 72 \mathrm{~h}^{8}\end{array}$ \\
\hline $\mathrm{ZnCl}_{2}$ & 53 & 13 & 23 & 90 & $\begin{array}{l}1.1 \text { equiv. metal salt } \\
2.0 \text { equiv. } \mathrm{Ac}_{2} \mathrm{O} \\
2.5 \text { equiv. pyridine } \\
\text { DMG, rt, } 72 \mathrm{~h}^{9}\end{array}$ \\
\hline & 80 & - & - & 80 & $\begin{array}{l}9.0 \text { equiv. "cat" } \\
1.4 \text { equiv. } \mathrm{Ac}_{2} \mathrm{O}, \\
\mathrm{DCM}, \mathrm{rt}, 18 \mathrm{~h}^{10}\end{array}$ \\
\hline wild type canadida rugosa liapse (CRL) & $>98$ & - & - & $>98$ & $\begin{array}{c}10 \mathrm{mg} \text { substrate, } \\
10 \mathrm{mg} \text { lipases, } \\
0.5 \mathrm{~mL} \text { vinyl acetate, } \\
\mathrm{rt}, 72 \mathrm{~h}^{11}\end{array}$ \\
\hline lipase PS (pseudomonas cepacian) & 85 & - & - & 85 & $\begin{array}{c}200 \mathrm{mg} \text { substrate, } \\
1 \mathrm{~g} \text { lipases absorbed } \\
\text { on celite, } \\
10 \mathrm{~mL} \text { vinyl acetate } \\
45^{\circ} \mathrm{C}, 7 \mathrm{~h}^{12}\end{array}$ \\
\hline
\end{tabular}

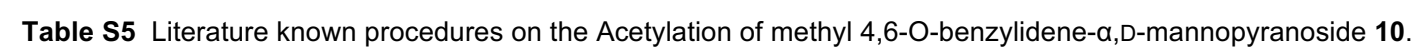

(2)




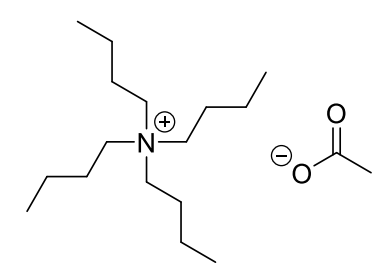

lipase PS (pseudomonas cepacian)
1 muiv.

$\mathrm{MeCN}, 40{ }^{\circ} \mathrm{C}, 12 \mathrm{~h}^{7}$

$200 \mathrm{mg}$ substrate,

$1 \mathrm{~g}$ lipases absorbed

97

$0 \mathrm{~mL}$ vinyl acetate

$20^{\circ} \mathrm{C}, 48 \mathrm{~h}^{12}$ 
NMR data

Methyl 4,6-O-methylidene- $\alpha, \mathrm{D}-$ glucopyranoside (4) $\left({ }^{1} \mathrm{H} 400 \mathrm{MHz},{ }^{13} \mathrm{C}\left\{{ }^{1} \mathrm{H}\right\} 100 \mathrm{MHz}, \mathrm{CDCl}_{3}\right)$

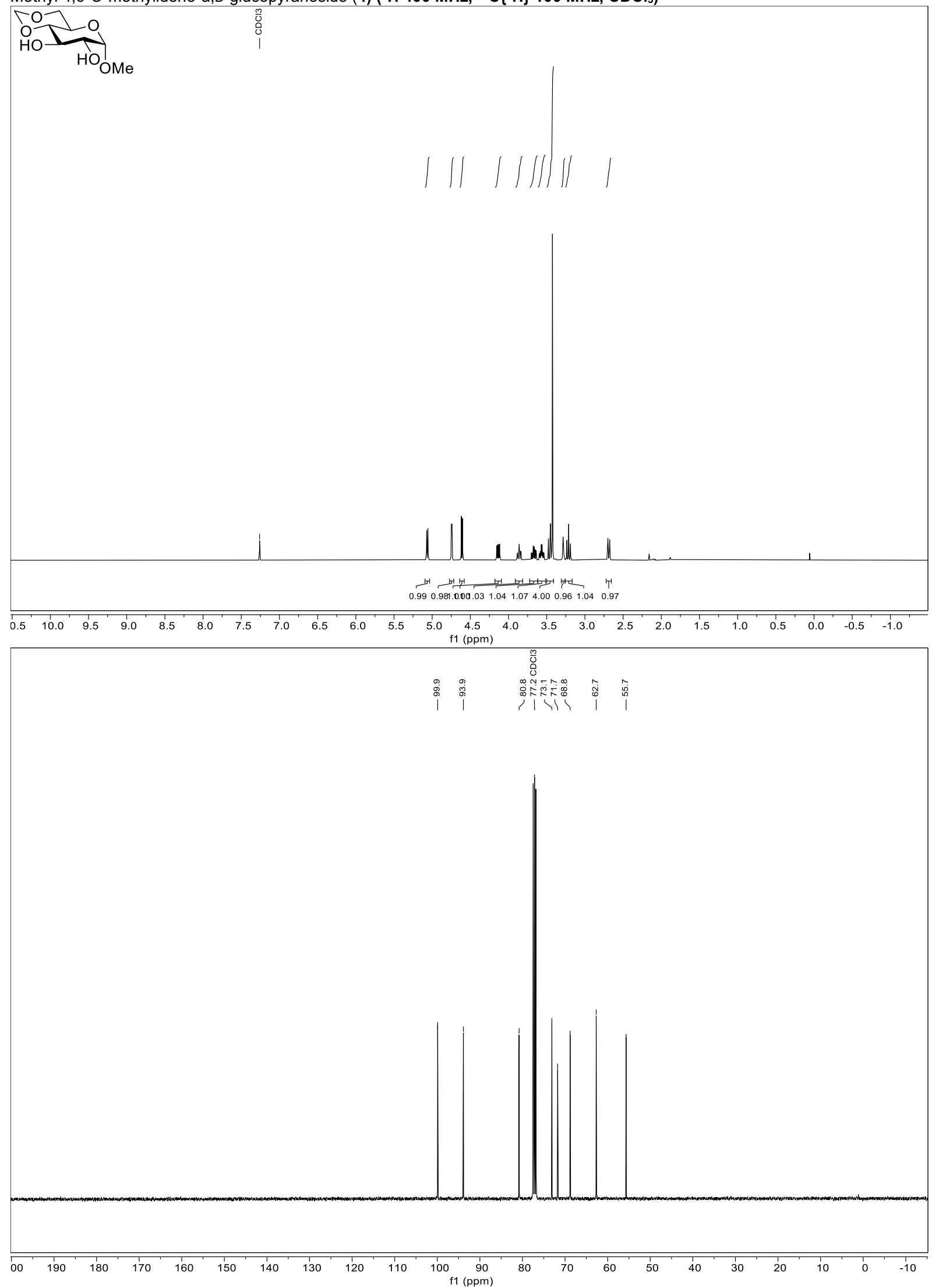


Methyl 4,6-O-cyclohexylidene- $\alpha$-D-glucopyranoside (5) $\left({ }^{1} \mathrm{H} 400 \mathrm{MHz},{ }^{13} \mathrm{C}\left\{{ }^{1} \mathrm{H}\right\} 100 \mathrm{MHz}, \mathrm{MeOH}-d_{4}\right)$

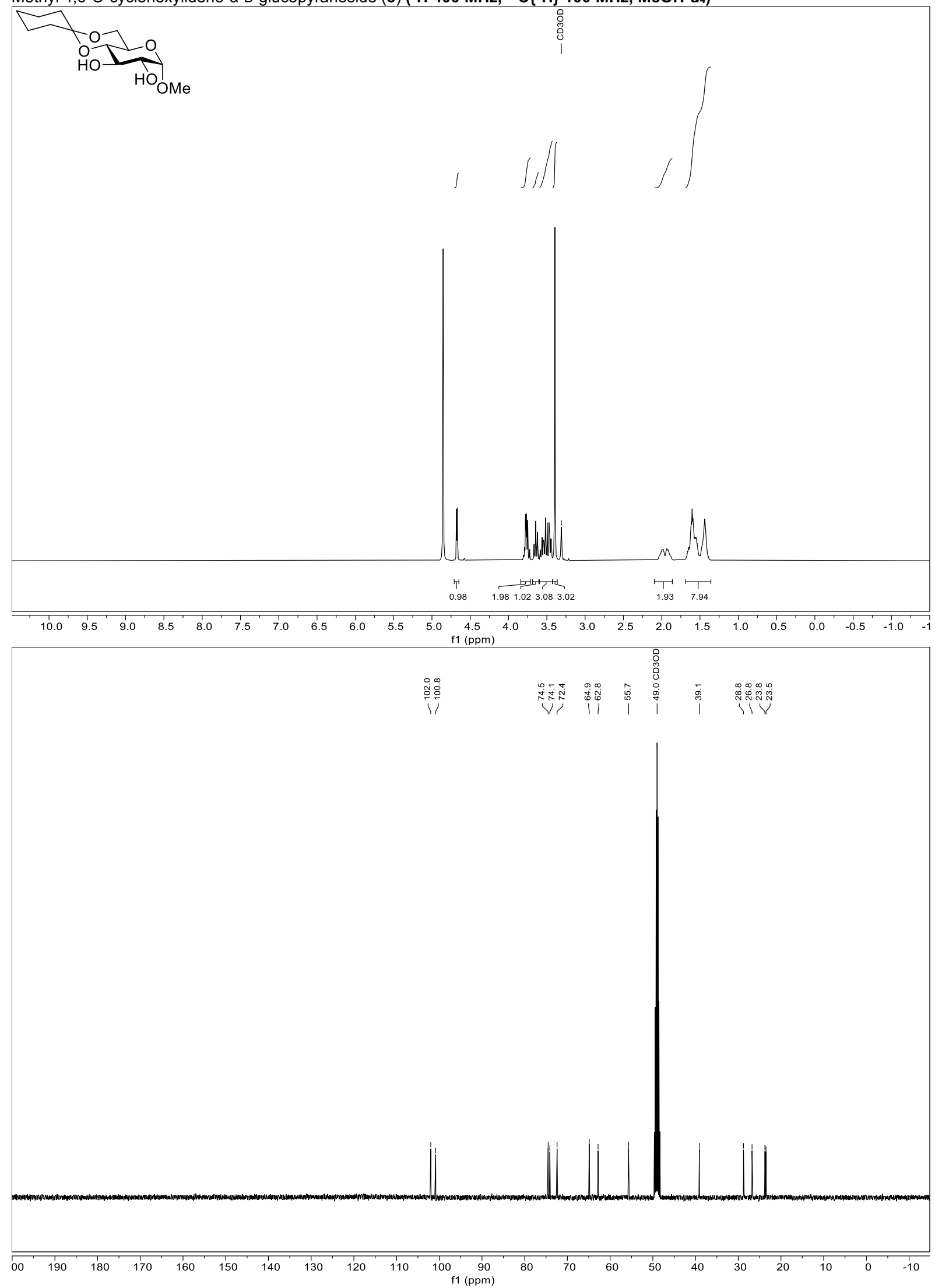


Methyl 4,6-O-benzylidene- $\beta$,D-glucopyranoside (6) $\left({ }^{1} \mathrm{H} 400 \mathrm{MHz},{ }^{13} \mathrm{C}\left\{{ }^{1} \mathrm{H}\right\} 100 \mathrm{MHz}\right.$, DMSO- $\left.d_{6}\right)$

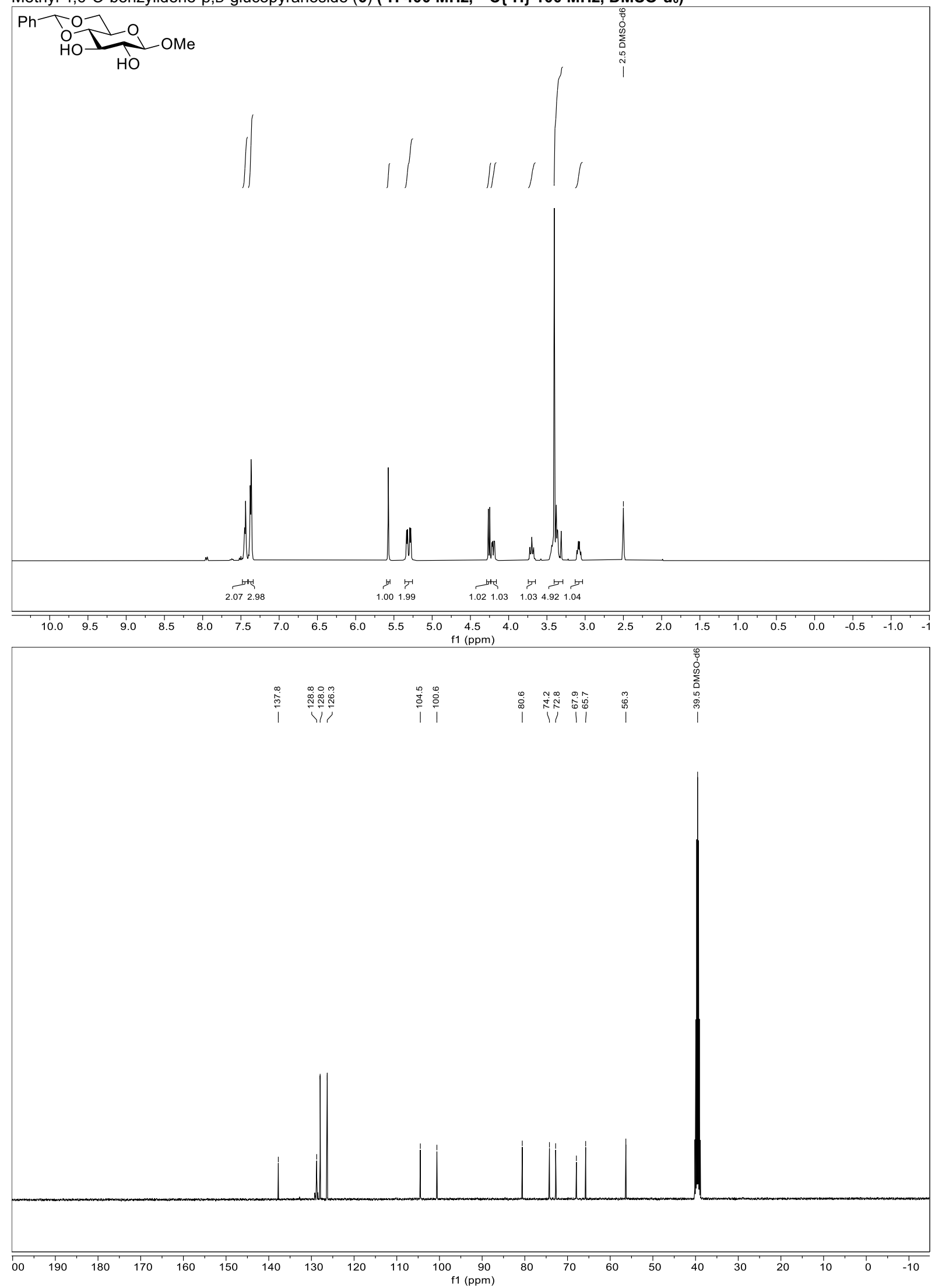


1,2,3,4,6-Pentaacetate-D-glucopyranose $(7 \mathrm{e})\left({ }^{1} \mathrm{H} 400 \mathrm{MHz},{ }^{13} \mathrm{C}\left\{{ }^{1} \mathrm{H}\right\} 100 \mathrm{MHz}, \mathrm{CDCl}_{3}\right)$

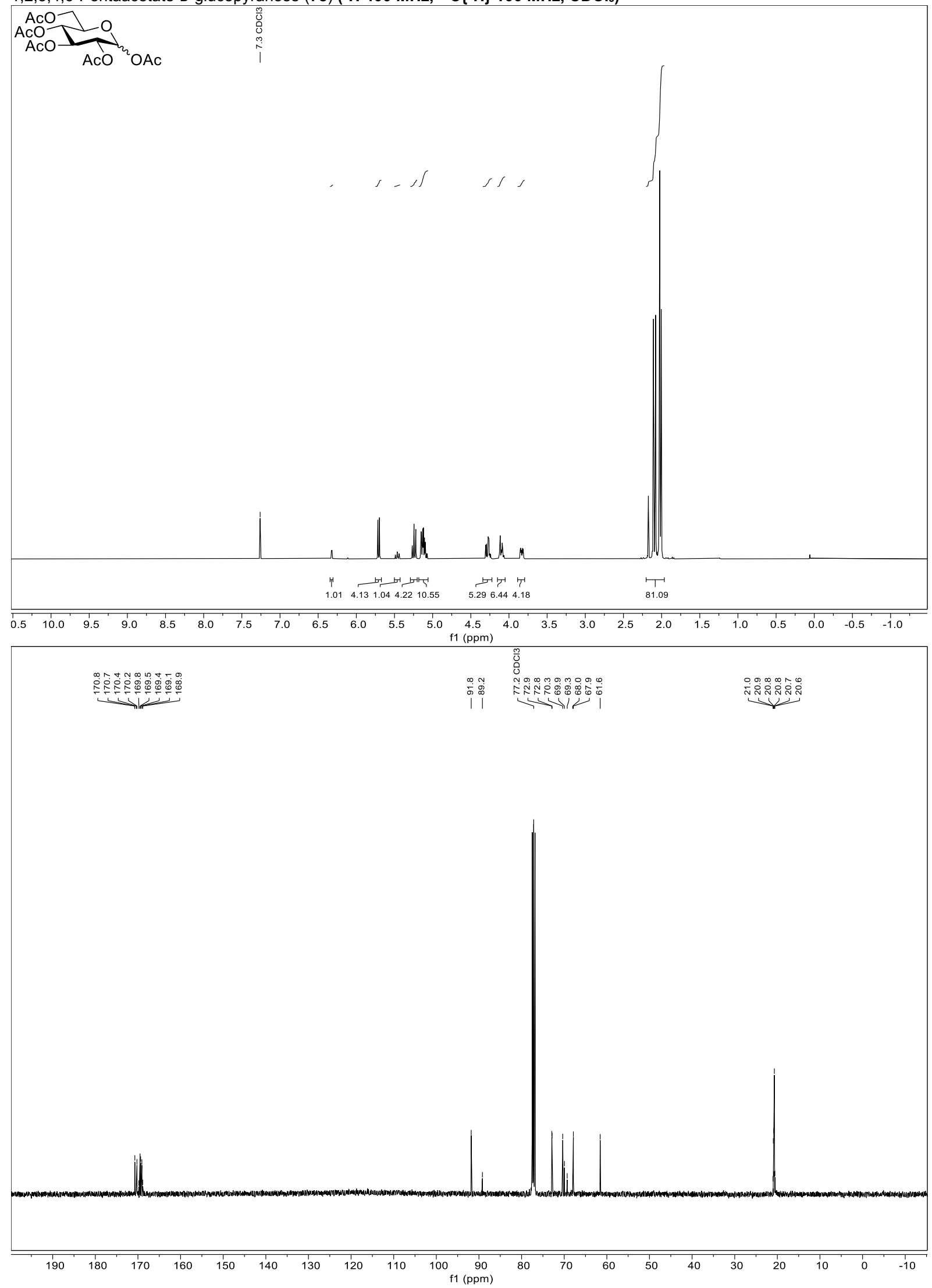


(4-methylphenyl)-1-thio-2,3,4,6-Pentaacetate- $\beta$,D-glucopyranoside $(7 \mathrm{f})\left({ }^{1} \mathrm{H} 400 \mathrm{MHz},{ }^{13} \mathrm{C}\left\{{ }^{1} \mathrm{H}\right\} 100 \mathrm{MHz}, \mathrm{CDCl}_{3}\right)$

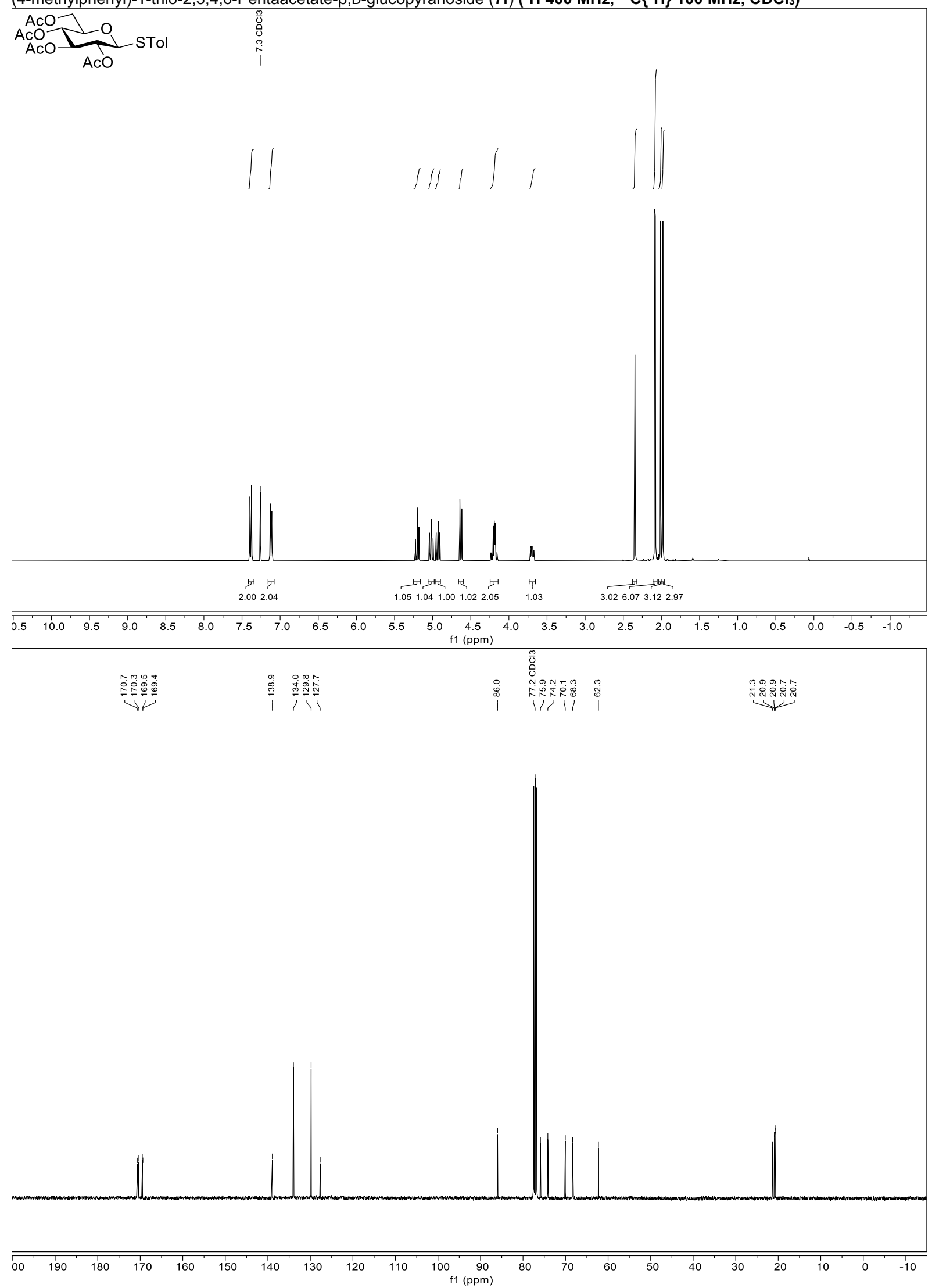


(4-methylphenyl)-4,6-O-benzylidene-1-thio- $\beta$,D-glucopyranoside (7) $\left({ }^{1} \mathrm{H} 400 \mathrm{MHz},{ }^{13} \mathrm{C}\left\{{ }^{1} \mathrm{H}\right\} 100 \mathrm{MHz}, \mathrm{CDCl}_{3}\right)$

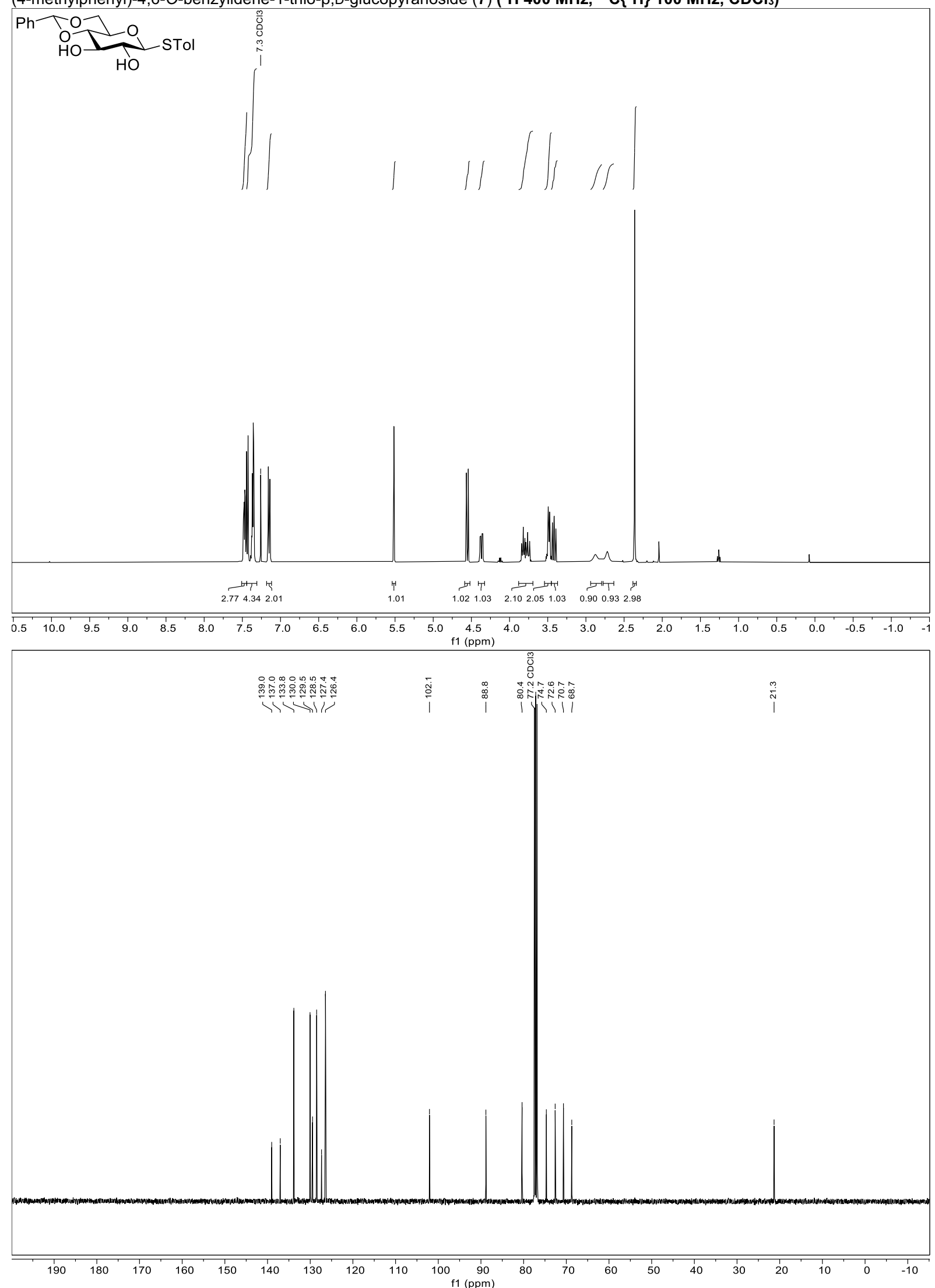


Methyl 4,6-O-benzylidene- $\alpha, \mathrm{D}-m a n n o p y r a n o s i d e ~(10) ~\left({ }^{1} \mathrm{H} 400 \mathrm{MHz},{ }^{13} \mathrm{C}\left\{{ }^{1} \mathrm{H}\right\} 100 \mathrm{MHz}, \mathrm{MeOH}-\mathrm{d}_{4}\right)$

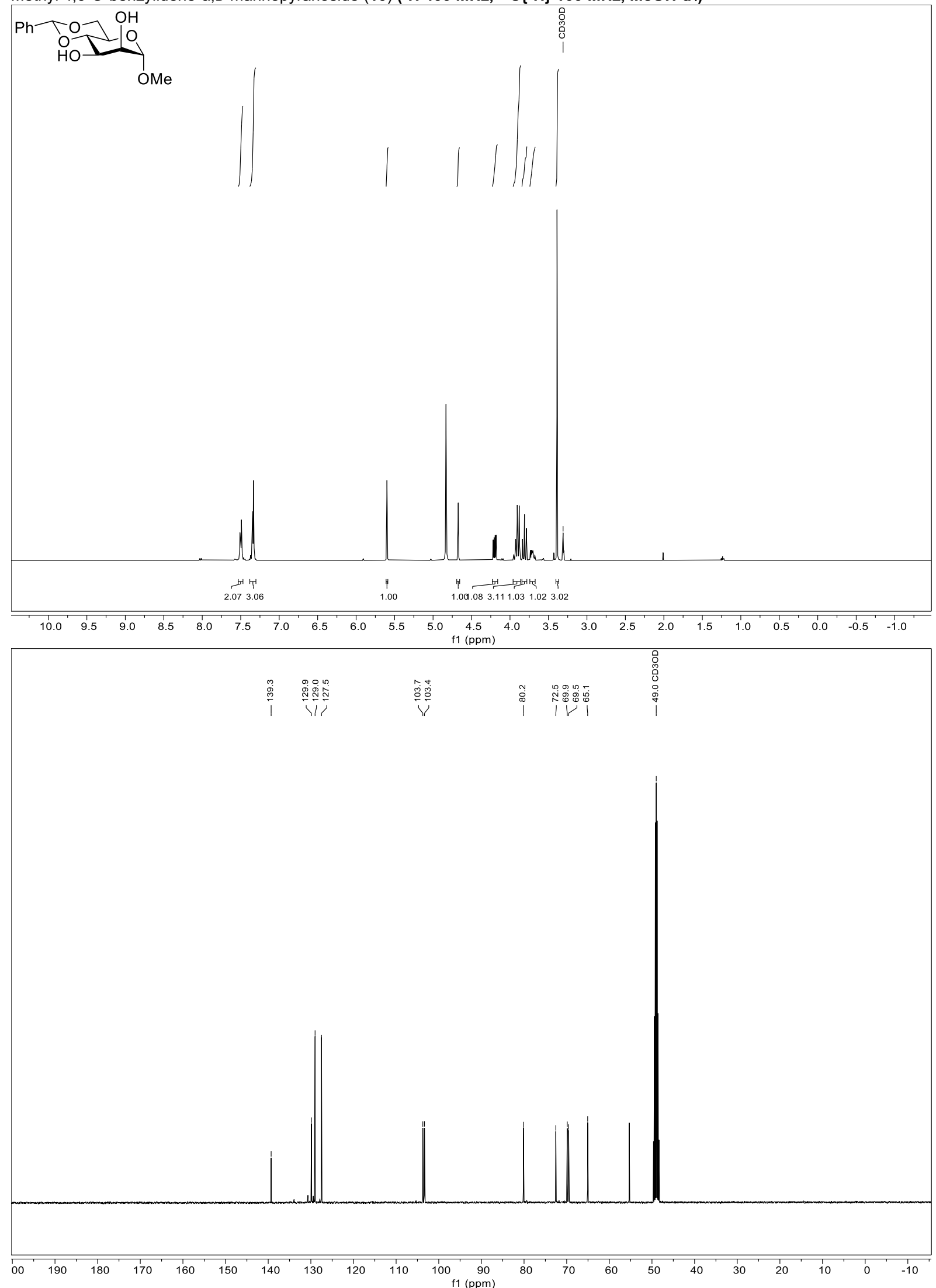


Methyl 4,6-O-methylidene- $\alpha, \mathrm{D}-\mathrm{mann} n \mathrm{p} y \mathrm{ranoside}(11)\left({ }^{1} \mathrm{H} 400 \mathrm{MHz},{ }^{13} \mathrm{C}\left\{{ }^{1} \mathrm{H}\right\} 100 \mathrm{MHz}, \mathrm{MeOH}-\mathrm{d}_{4}\right)$

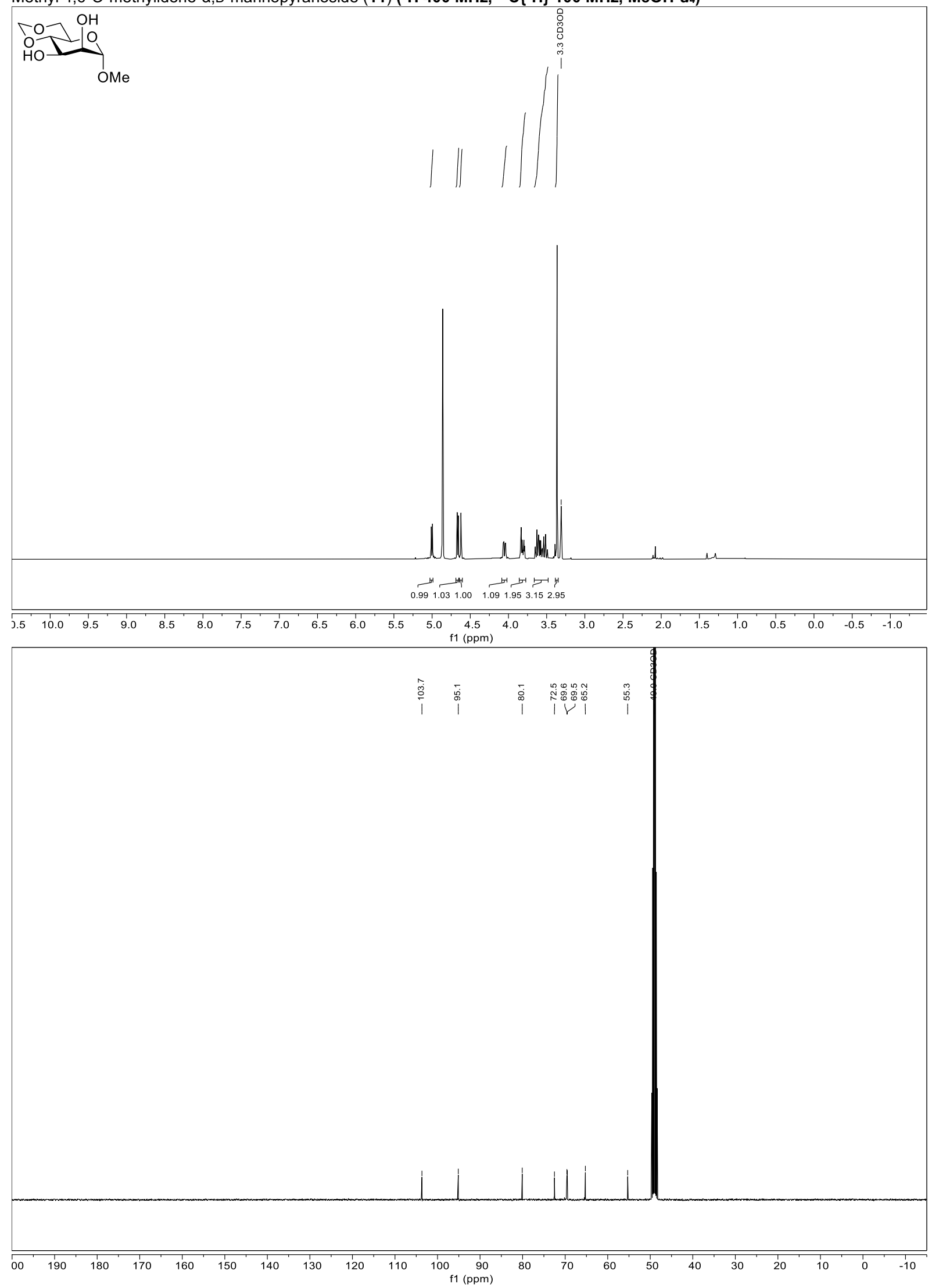


Methyl 4,6-O-(2-naphtylidene)-a,D-mannopyranoside (12) $\left({ }^{1} \mathrm{H} 400 \mathrm{MHz},{ }^{13} \mathrm{C}\left\{{ }^{1} \mathrm{H}\right\} 100 \mathrm{MHz}, \mathrm{MeOH}-\mathrm{d}_{4}\right)$
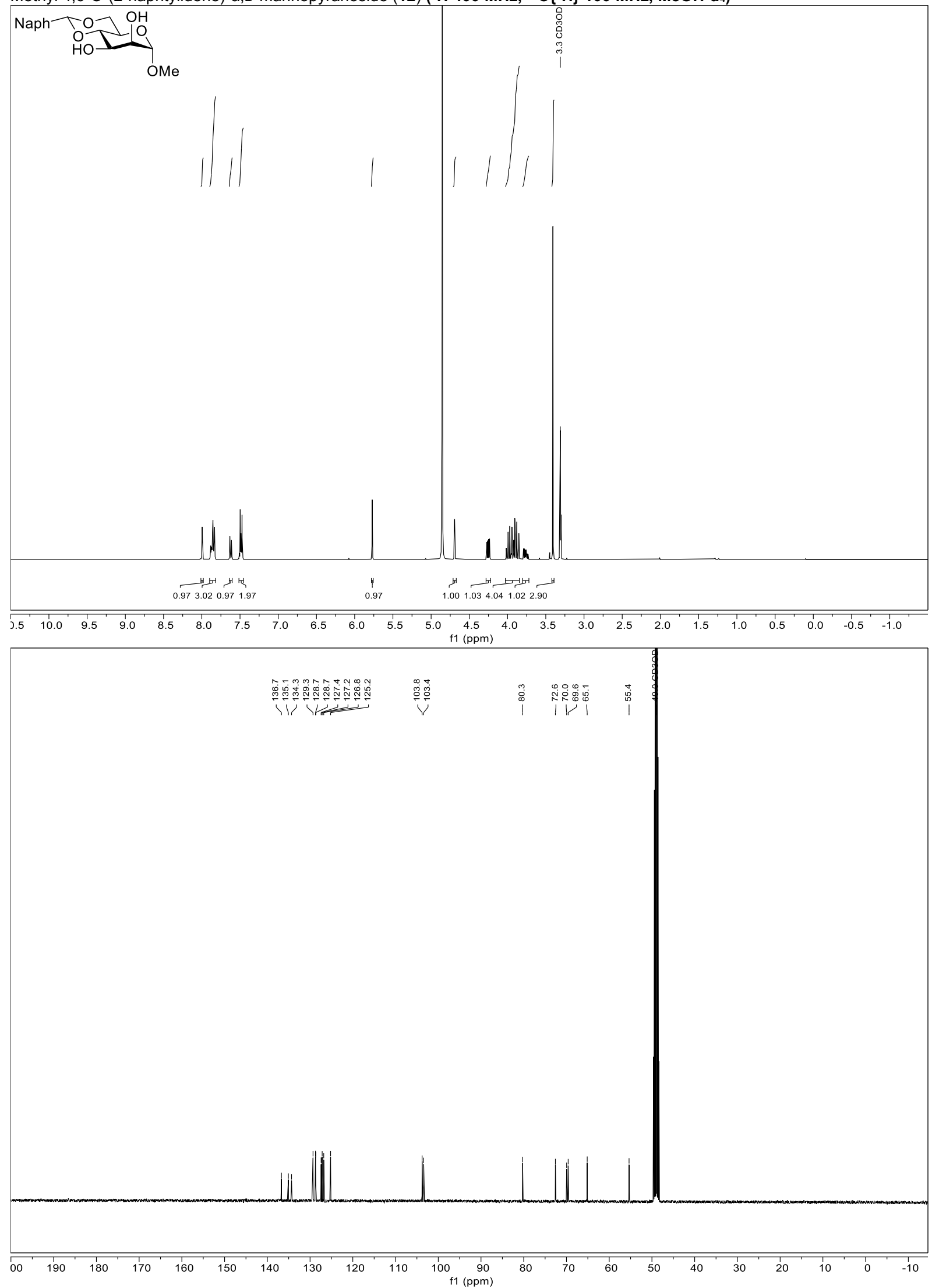
Methyl 4,6-O-benzylidene- $\alpha, \mathrm{D}-$ galactopyranoside (13) $\left({ }^{1} \mathrm{H} 400 \mathrm{MHz},{ }^{13} \mathrm{C}\left\{{ }^{1} \mathrm{H}\right\} 100 \mathrm{MHz}, \mathrm{CDCl}_{3}\right)$

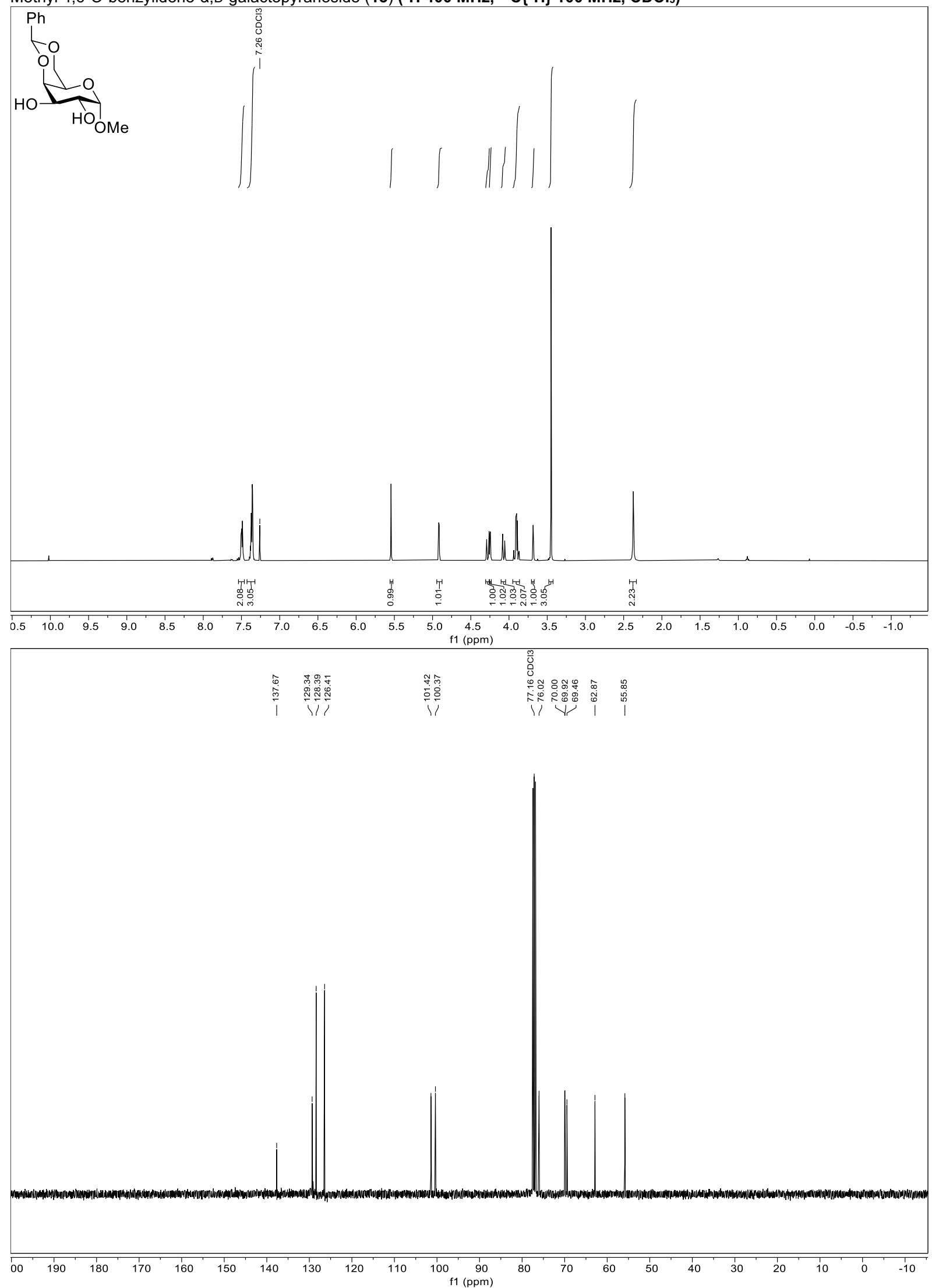


Methyl 2-O-acetyl-4,6-O-benzylidene- $\alpha, \mathrm{D}$-glucopyranoside (3a) $\left({ }^{1} \mathrm{H} 400 \mathrm{MHz},{ }^{13} \mathrm{C}\left\{{ }^{1} \mathrm{H}\right\} 100 \mathrm{MHz}, \mathrm{CDCl}_{3}\right)$
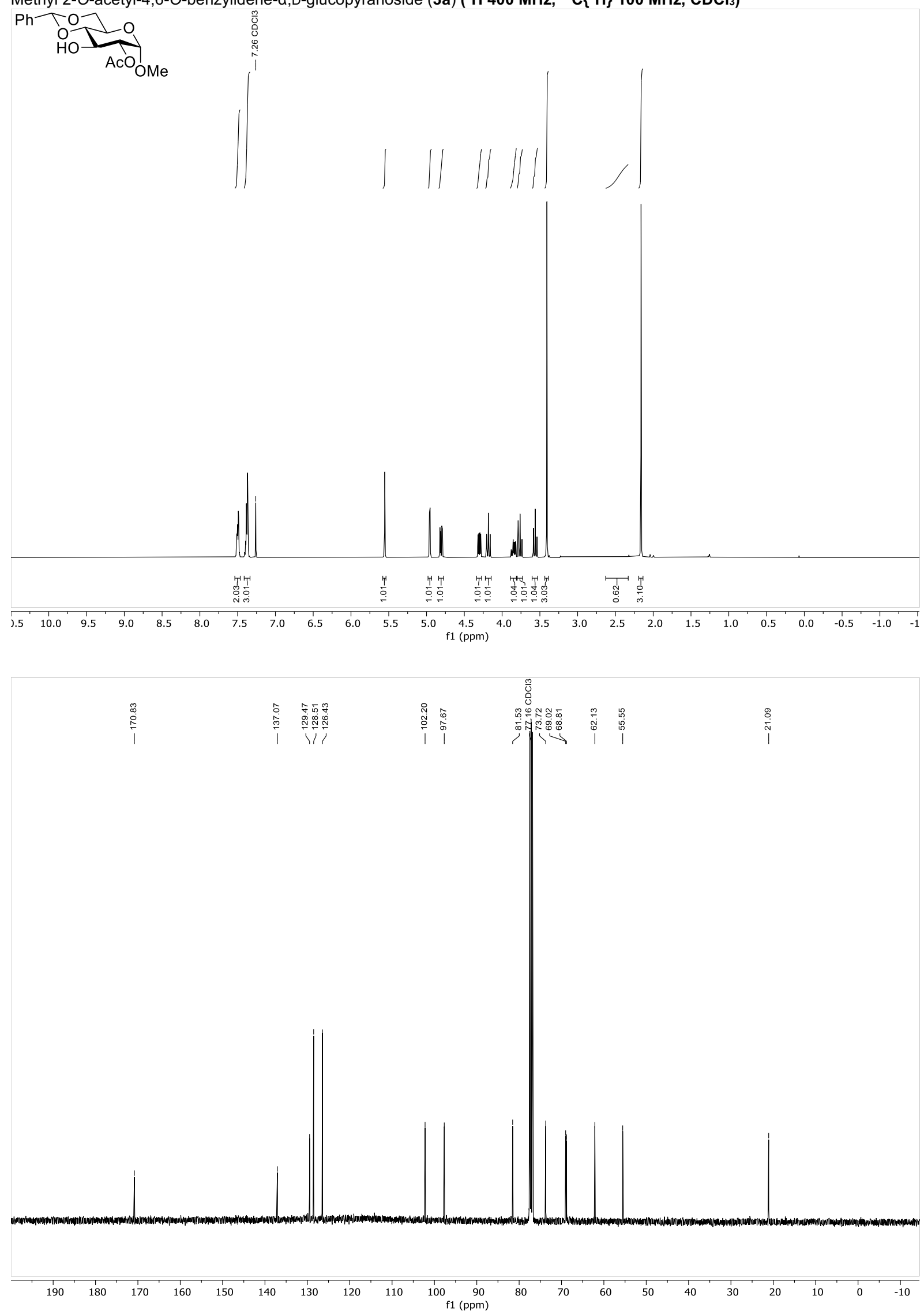
Methyl 3-O-acetyl-4,6-O-benzylidene-, ,D-glucopyranoside (3b) $\left({ }^{1} \mathrm{H} 400 \mathrm{MHz},{ }^{13} \mathrm{C}\left\{{ }^{1} \mathrm{H}\right\} 100 \mathrm{MHz}, \mathrm{CDCl}_{3}\right)$
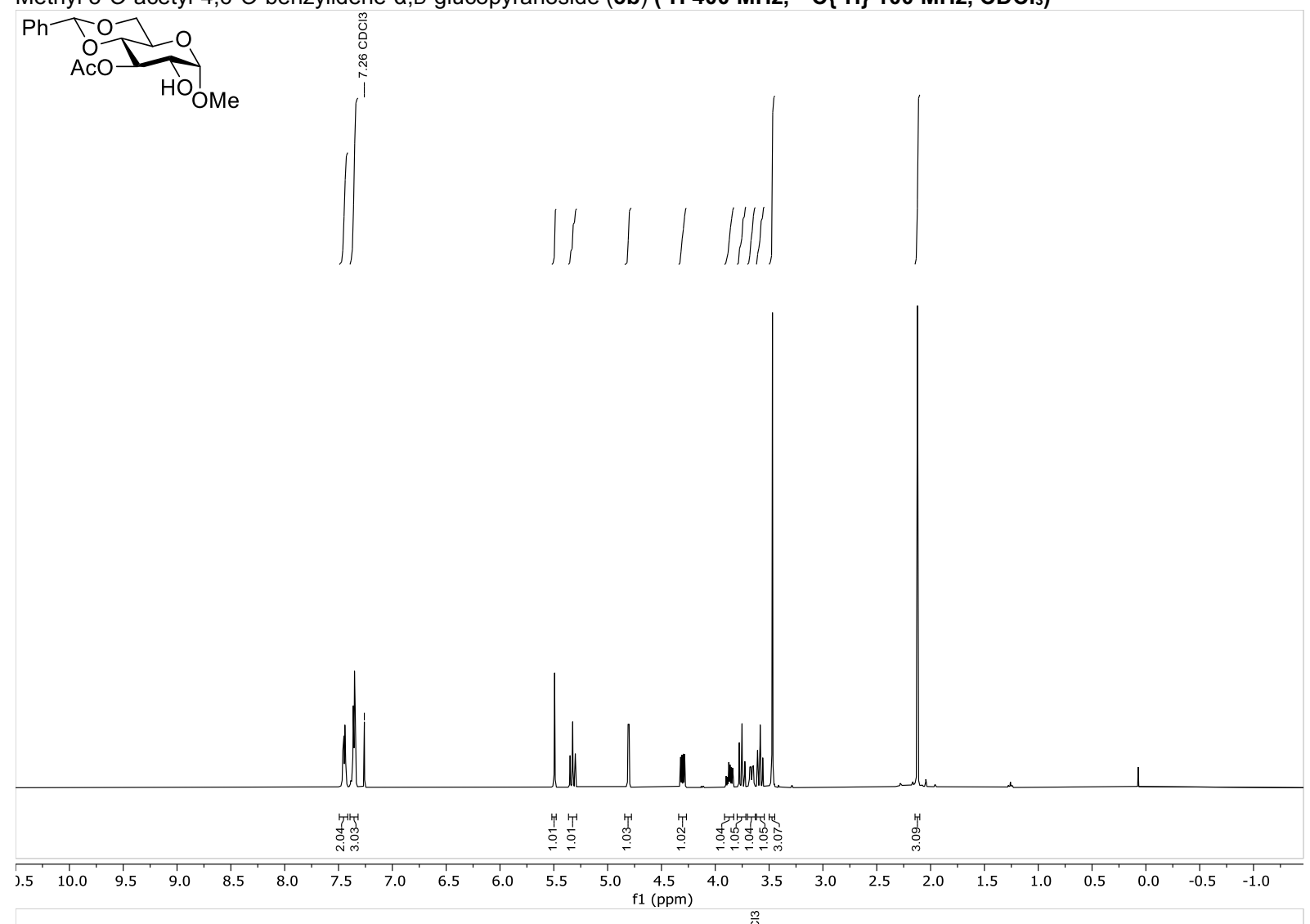

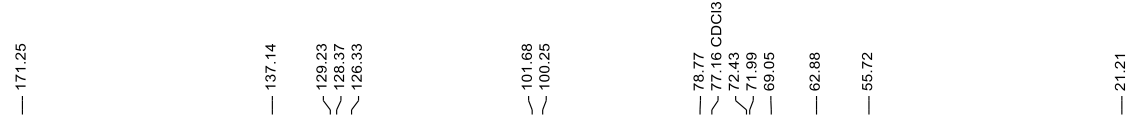

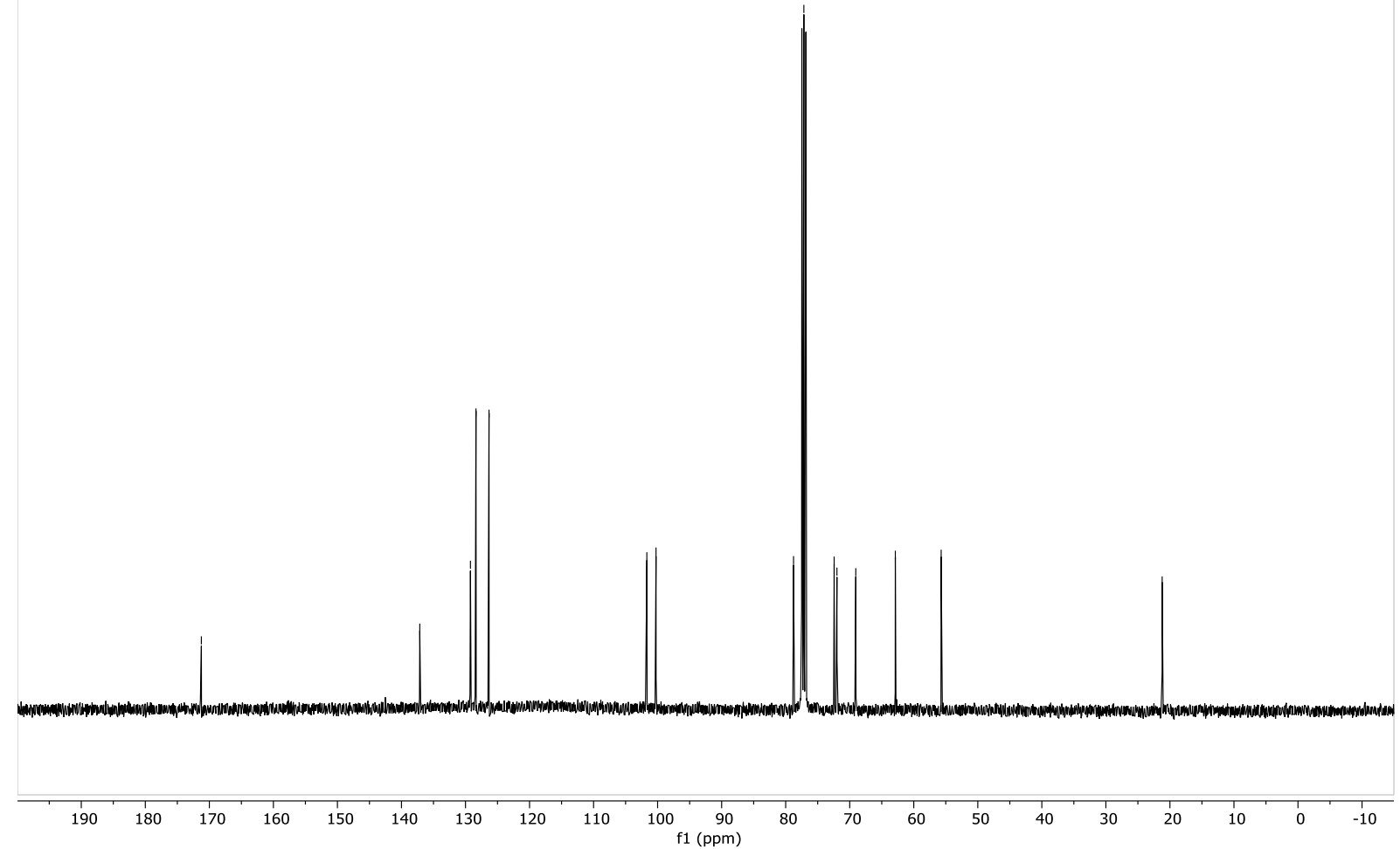

$\begin{array}{llllllllll}190 & 180 & 170 & 160 & 150 & 140 & 130 & 120 & 110 & 100 \begin{array}{r}90 \\ \mathrm{f} 1(\mathrm{ppm})\end{array}\end{array}$

$\frac{1}{\stackrel{4}{4}}$ 

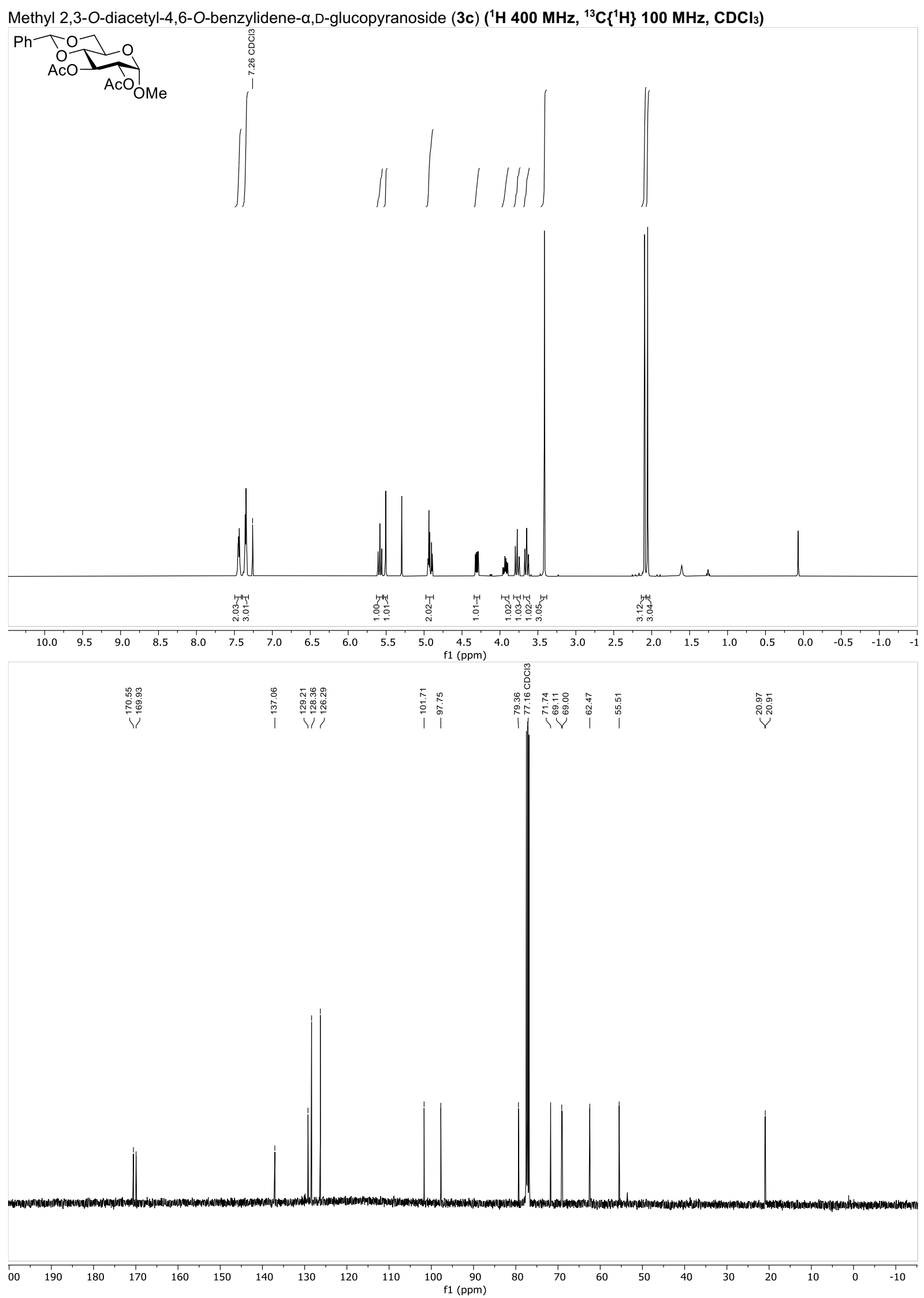

S35 
Methyl 2-O-acetyl-4,6-O-methylidene- $\alpha, \mathrm{D}-$ glucopyranoside $(4 \mathrm{a})\left({ }^{1} \mathrm{H} 600 \mathrm{MHz},{ }^{13} \mathrm{C}\left\{{ }^{1} \mathrm{H}\right\} 150 \mathrm{MHz}, \mathrm{CDCl}_{3}\right)$
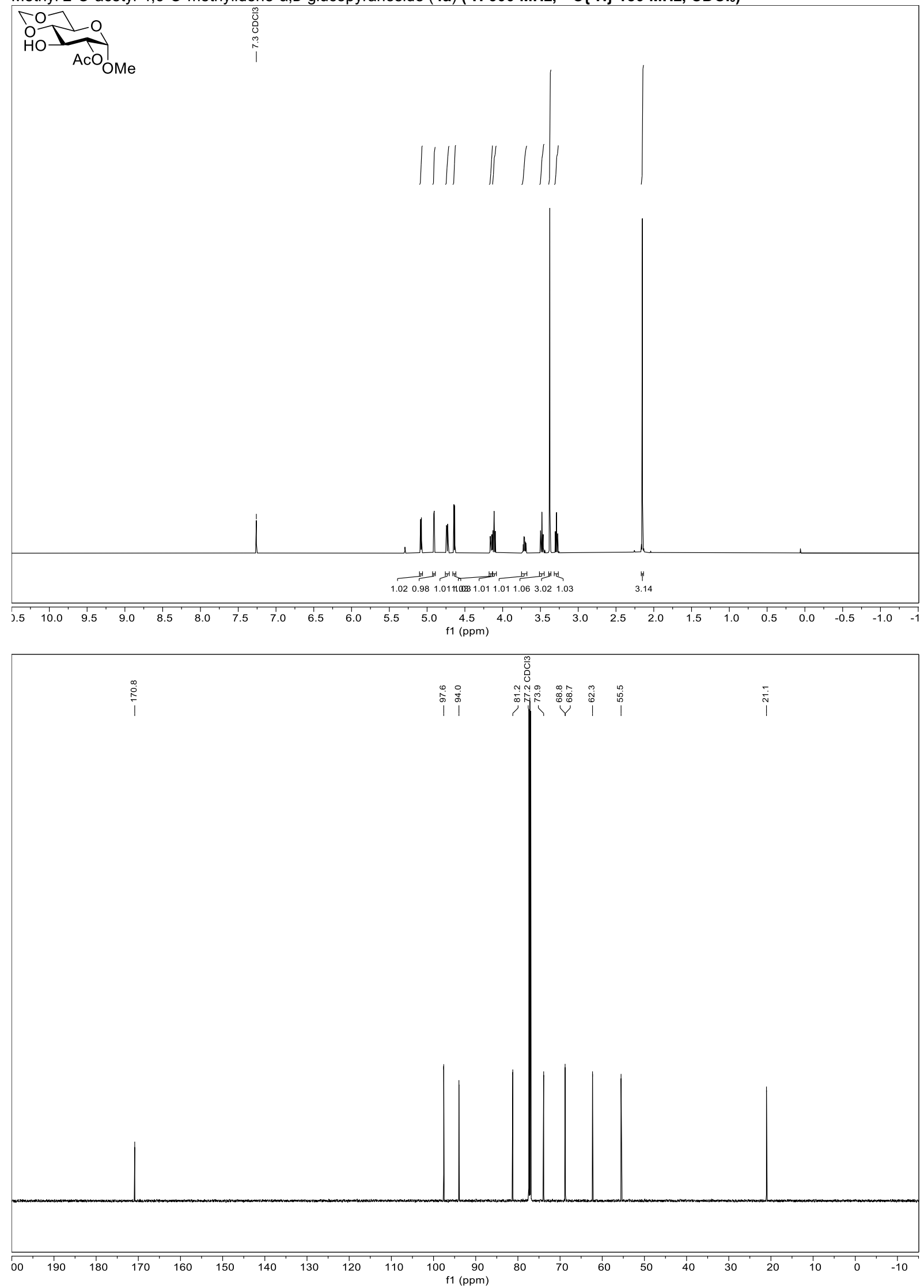
Methyl 3-O-acetyl-4,6-O-methylidene- $\alpha, \mathrm{D}-$ glucopyranoside (4b) $\left({ }^{1} \mathrm{H} 600 \mathrm{MHz},{ }^{13} \mathrm{C}\left\{{ }^{1} \mathrm{H}\right\} 150 \mathrm{MHz}, \mathrm{CDCl}{ }_{3}\right)$
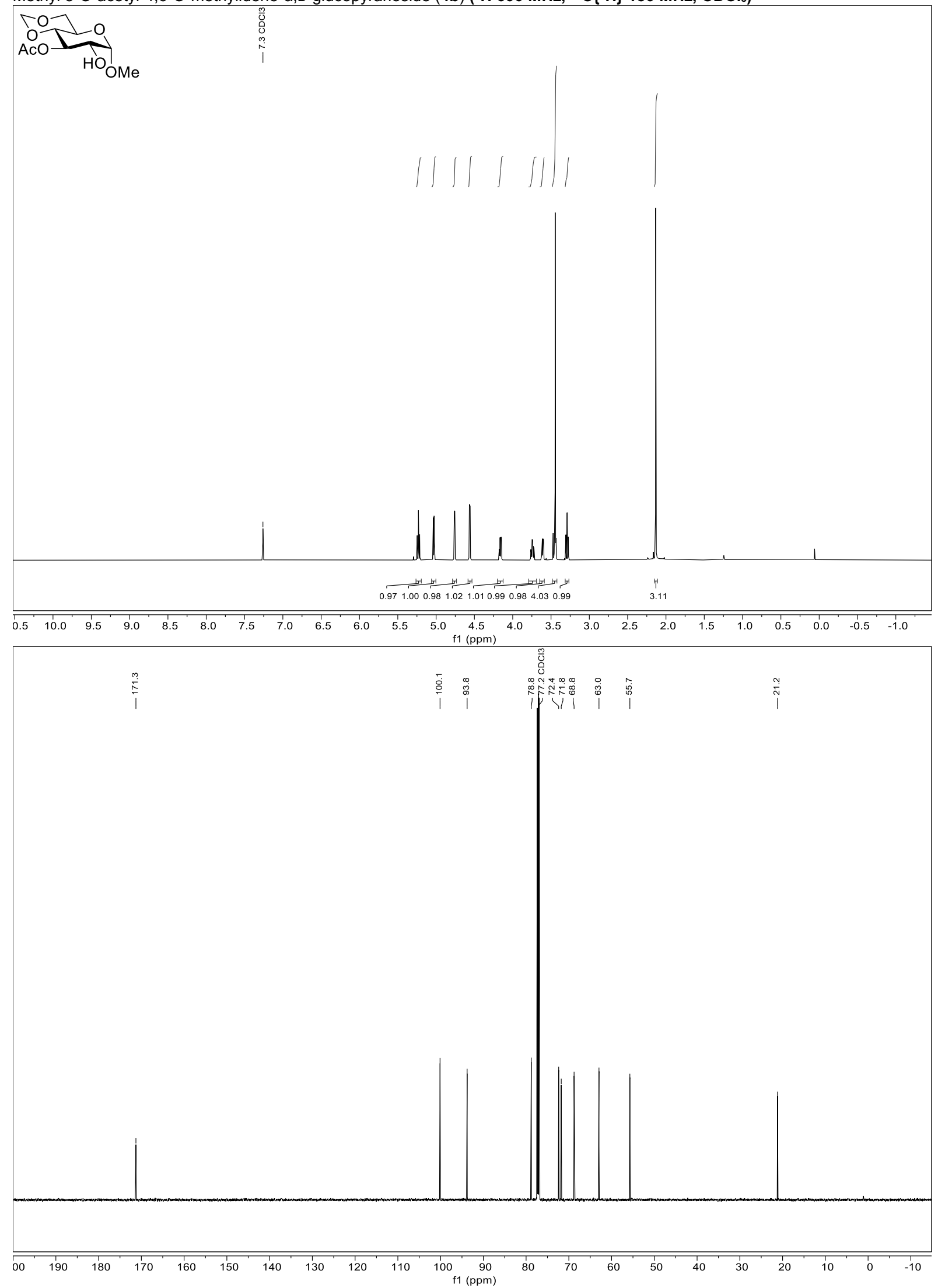
Methyl 2,3-O-diacetyl-4,6-O-methylidene- $\alpha, \mathrm{D}-$ glucopyranoside $(4 \mathrm{c})\left({ }^{1} \mathrm{H} 400 \mathrm{MHz},{ }^{13} \mathrm{C}\left\{{ }^{1} \mathrm{H}\right\} 100 \mathrm{MHz}, \mathrm{CDCl}_{3}\right)$

$$
\text { }
$$
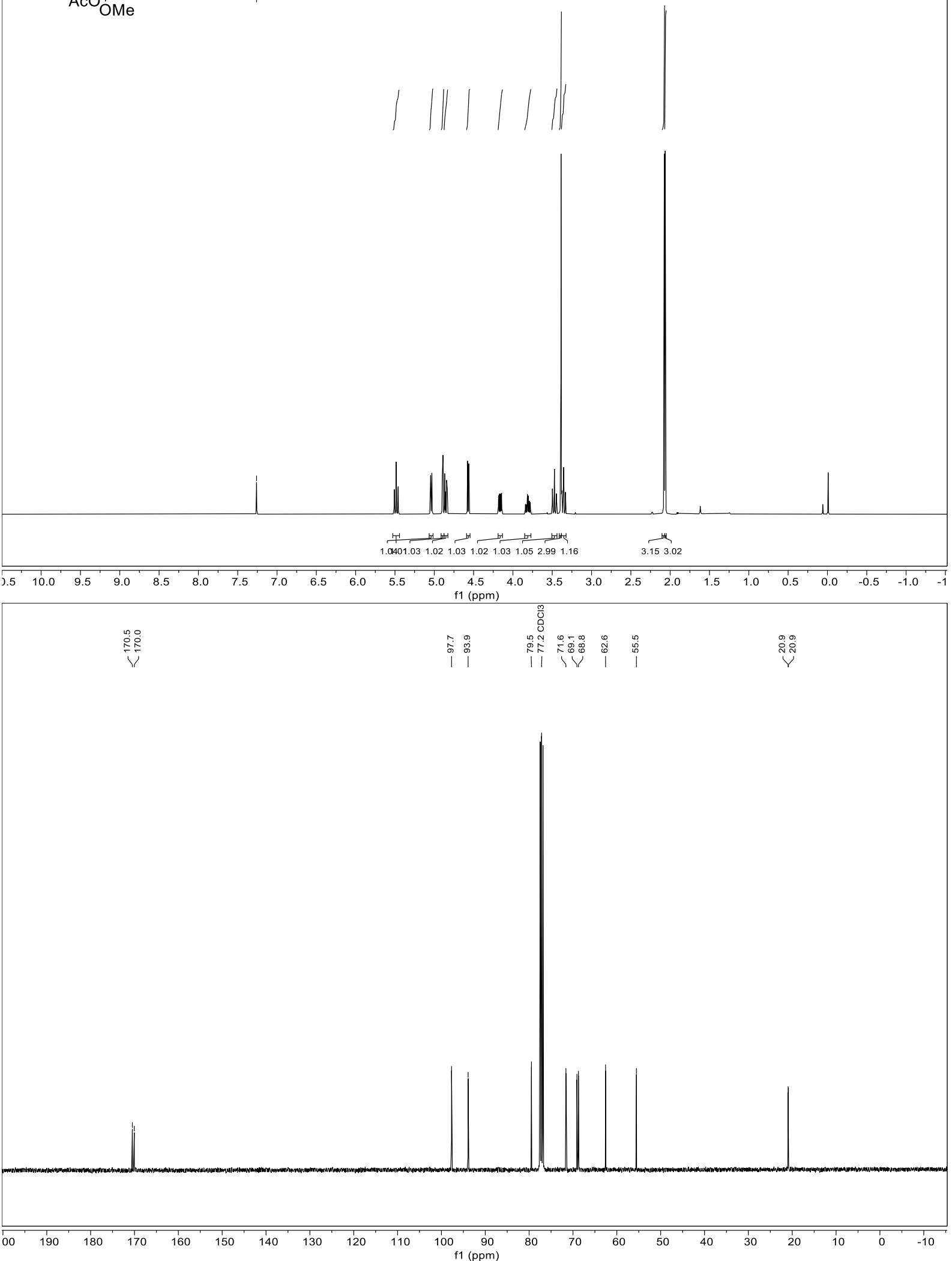
Methyl 2-O-acetyl-4,6-O-cyclohexylidene- $\alpha, \mathrm{D}-$ glucopyranoside $(5 \mathrm{a})\left({ }^{1} \mathrm{H} 400 \mathrm{MHz},{ }^{13} \mathrm{C}\left\{{ }^{1} \mathrm{H}\right\} 100 \mathrm{MHz}, \mathrm{MeOH}-\mathrm{d}_{4}\right)$

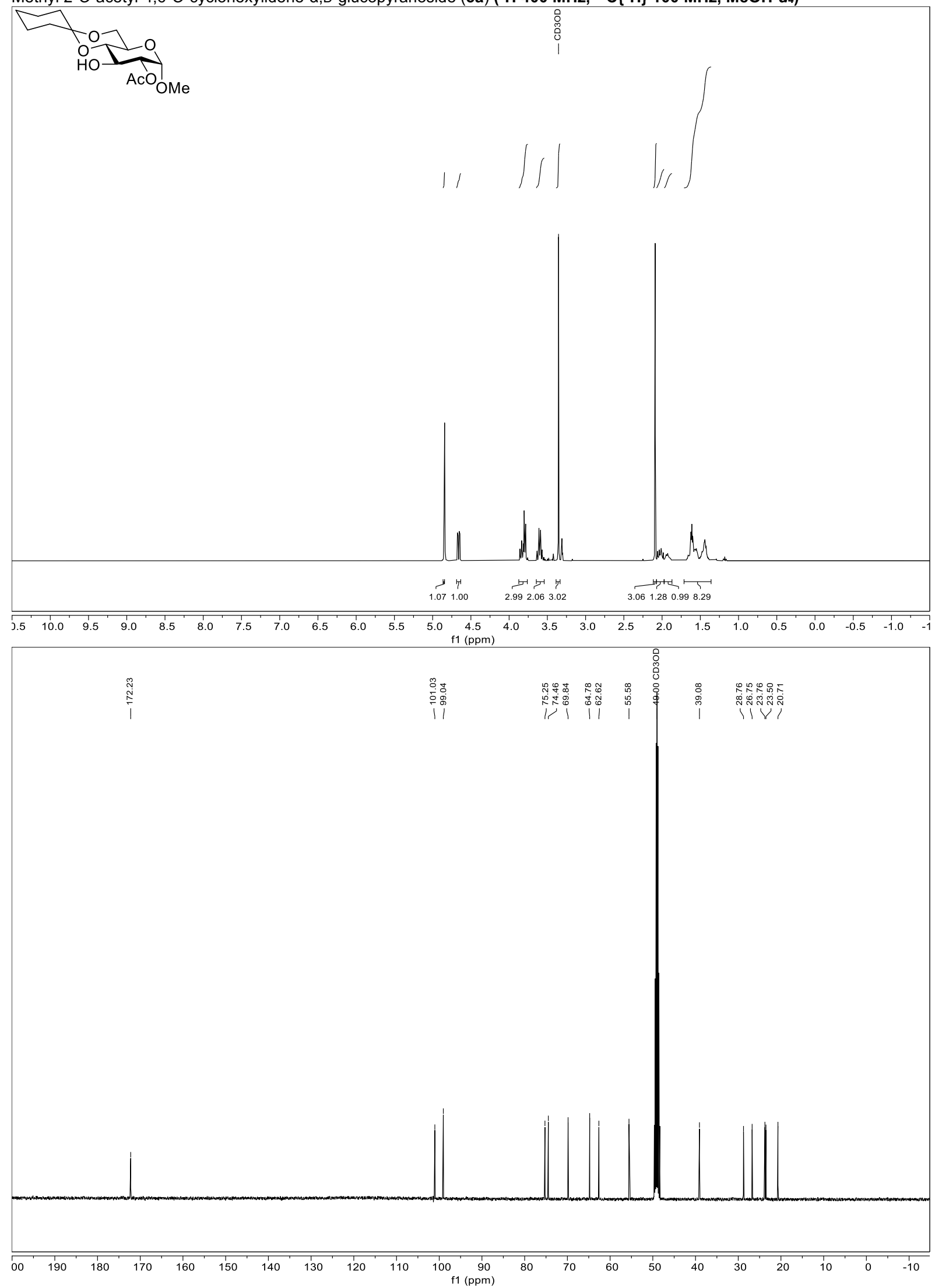



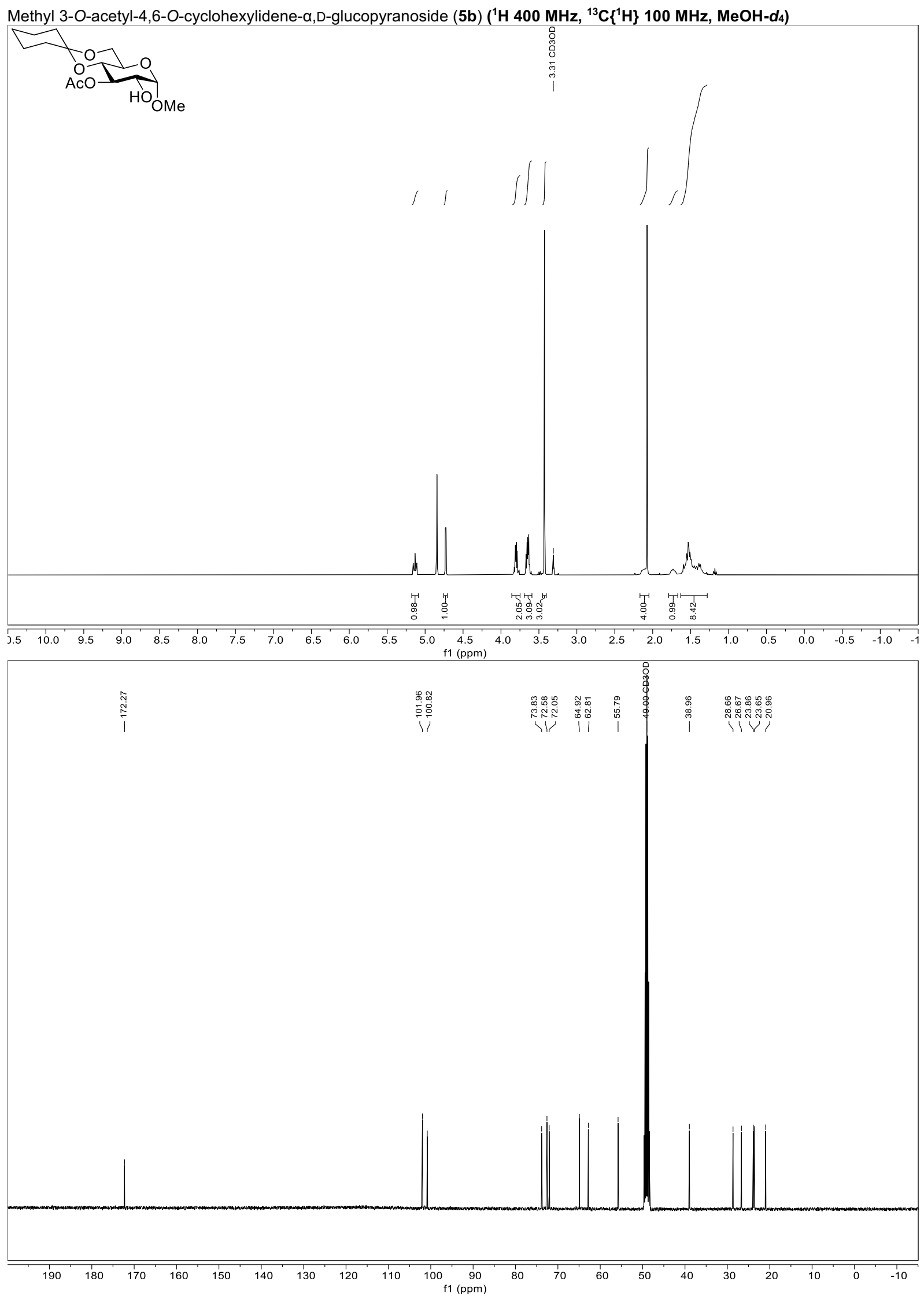

S40 
Methyl 2,3-O-diacetyl-4,6-O-cyclohexylidene- $\alpha, \mathrm{D}-$ glucopyranoside $(5 \mathrm{C})\left({ }^{1} \mathrm{H} 400 \mathrm{MHz},{ }^{13} \mathrm{C}\left\{{ }^{1} \mathrm{H}\right\} 100 \mathrm{MHz}, \mathrm{MeOH}-d_{4}\right)$
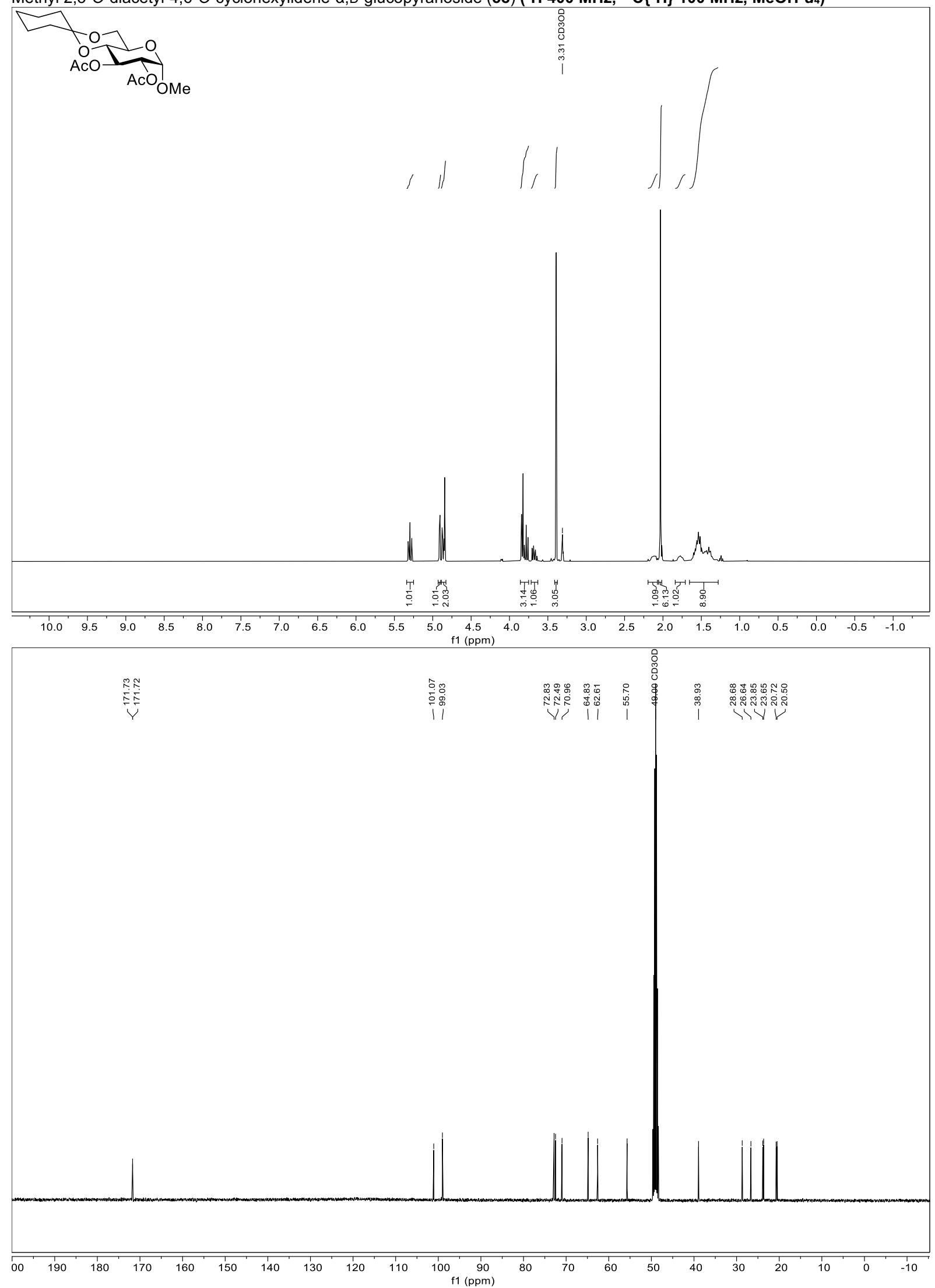
Methyl 2-O-acetyl-4,6-O-benzylidene- $\beta$,D-glucopyranoside (6a) $\left({ }^{1} \mathrm{H} 400 \mathrm{MHz},{ }^{13} \mathrm{C}\left\{{ }^{1} \mathrm{H}\right\} 100 \mathrm{MHz}\right.$, DMSO-d 6 )
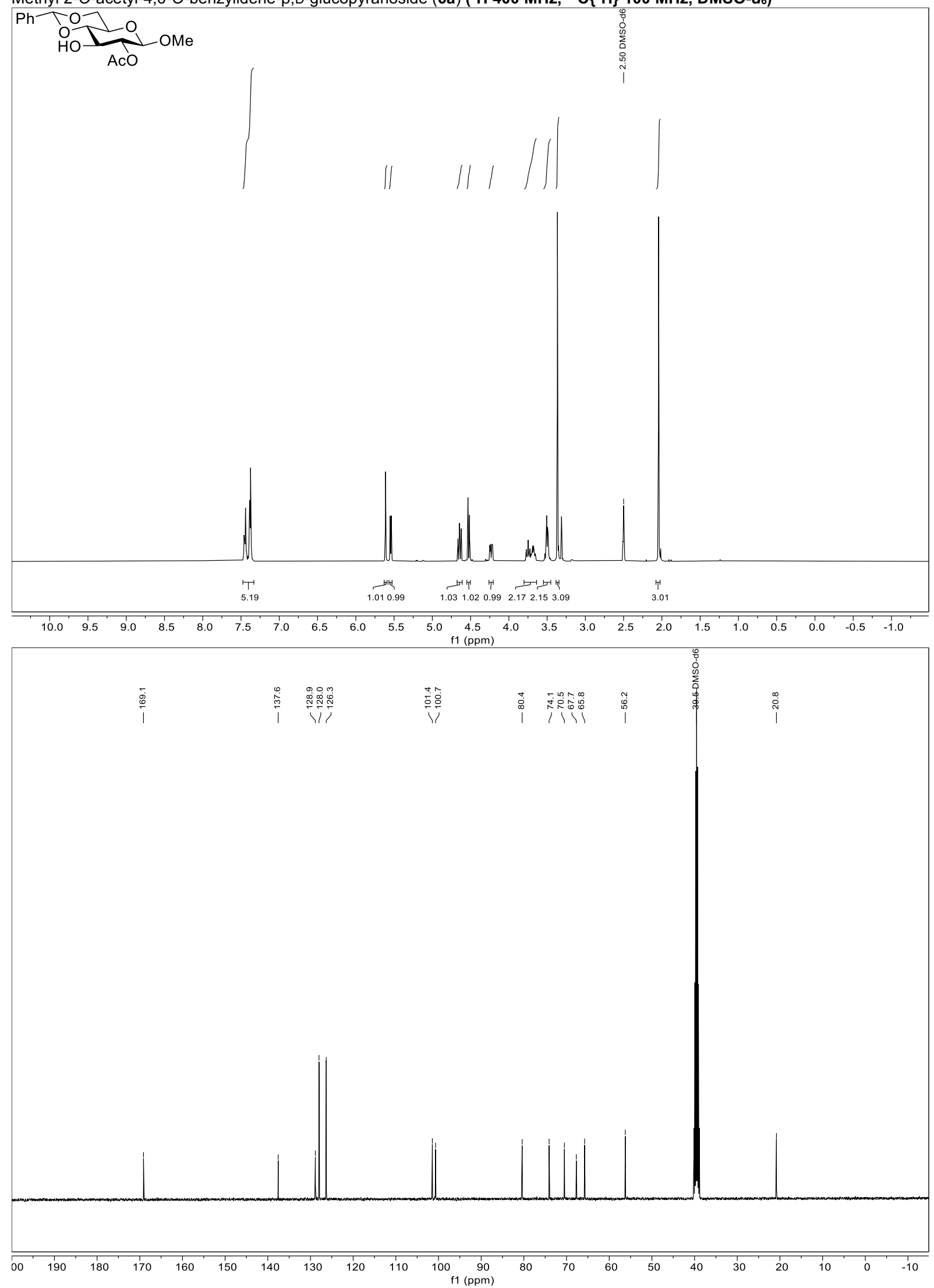
Methyl 3-O-acetyl-4,6-O-benzylidene- $\beta$,D-glucopyranoside $(6 \mathrm{~b})\left({ }^{1} \mathrm{H} 400 \mathrm{MHz},{ }^{13} \mathrm{C}\left\{{ }^{1} \mathrm{H}\right\} 100 \mathrm{MHz}, \mathrm{DMSO}-\mathrm{d}_{6}\right)$
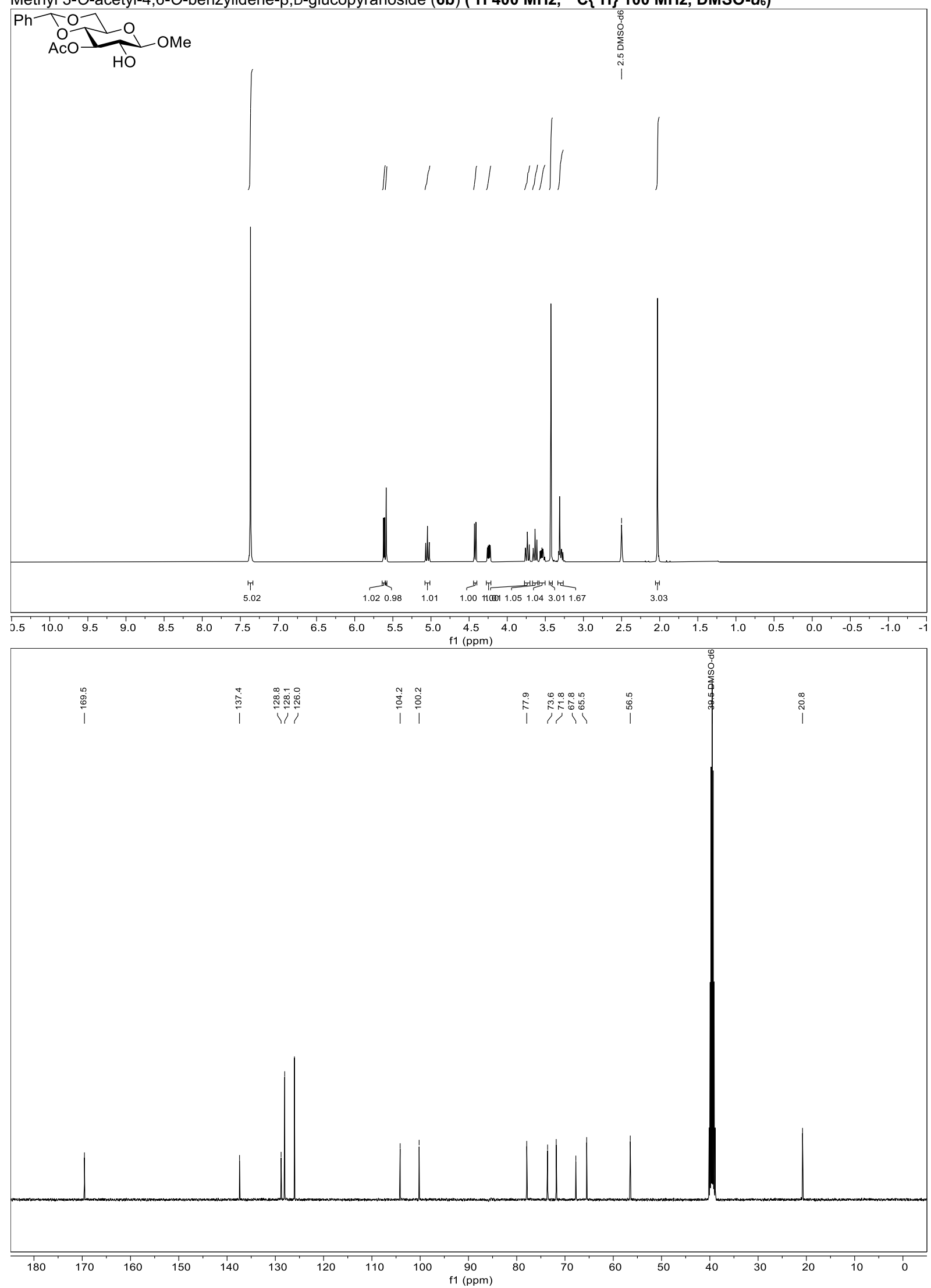
Methyl 2,3-O-diacetyl-4,6-O-benzylidene- $\beta$,D-glucopyranoside (6c) $\left({ }^{1} \mathrm{H} 400 \mathrm{MHz},{ }^{13} \mathrm{C}\left\{{ }^{1} \mathrm{H}\right\} 100 \mathrm{MHz}, \mathrm{DMSO}-d_{6}\right)$
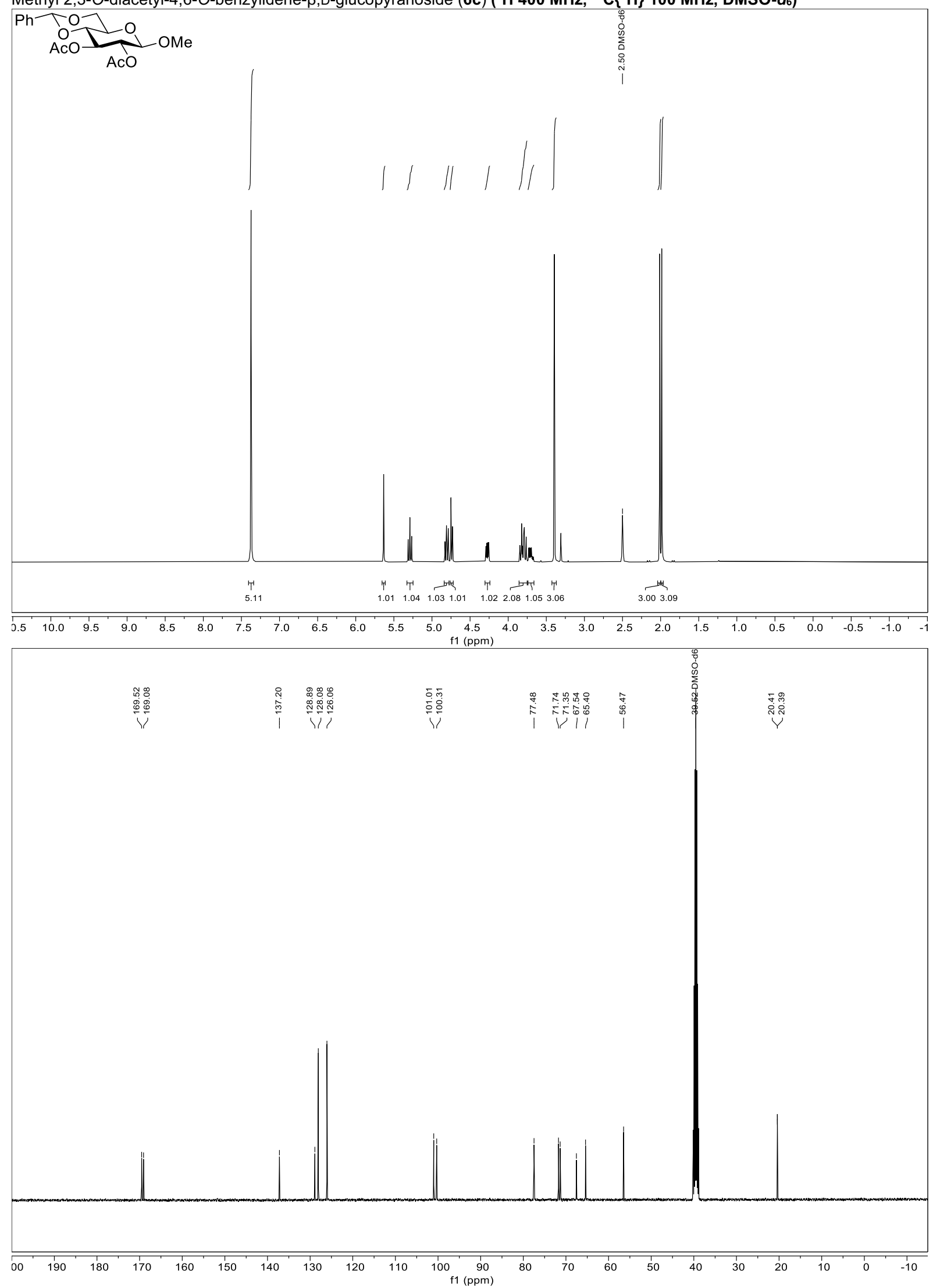
4-Methylphenyl 2-O-acetyl-4,6-O-benzylidene-1-thio- $\beta$,D-glucopyranoside $(7 \mathrm{a})\left({ }^{1} \mathrm{H} 400 \mathrm{MHz},{ }^{13} \mathrm{C}\left\{{ }^{1} \mathrm{H}\right\} 100 \mathrm{MHz}, \mathrm{CDCl}_{3}\right)$
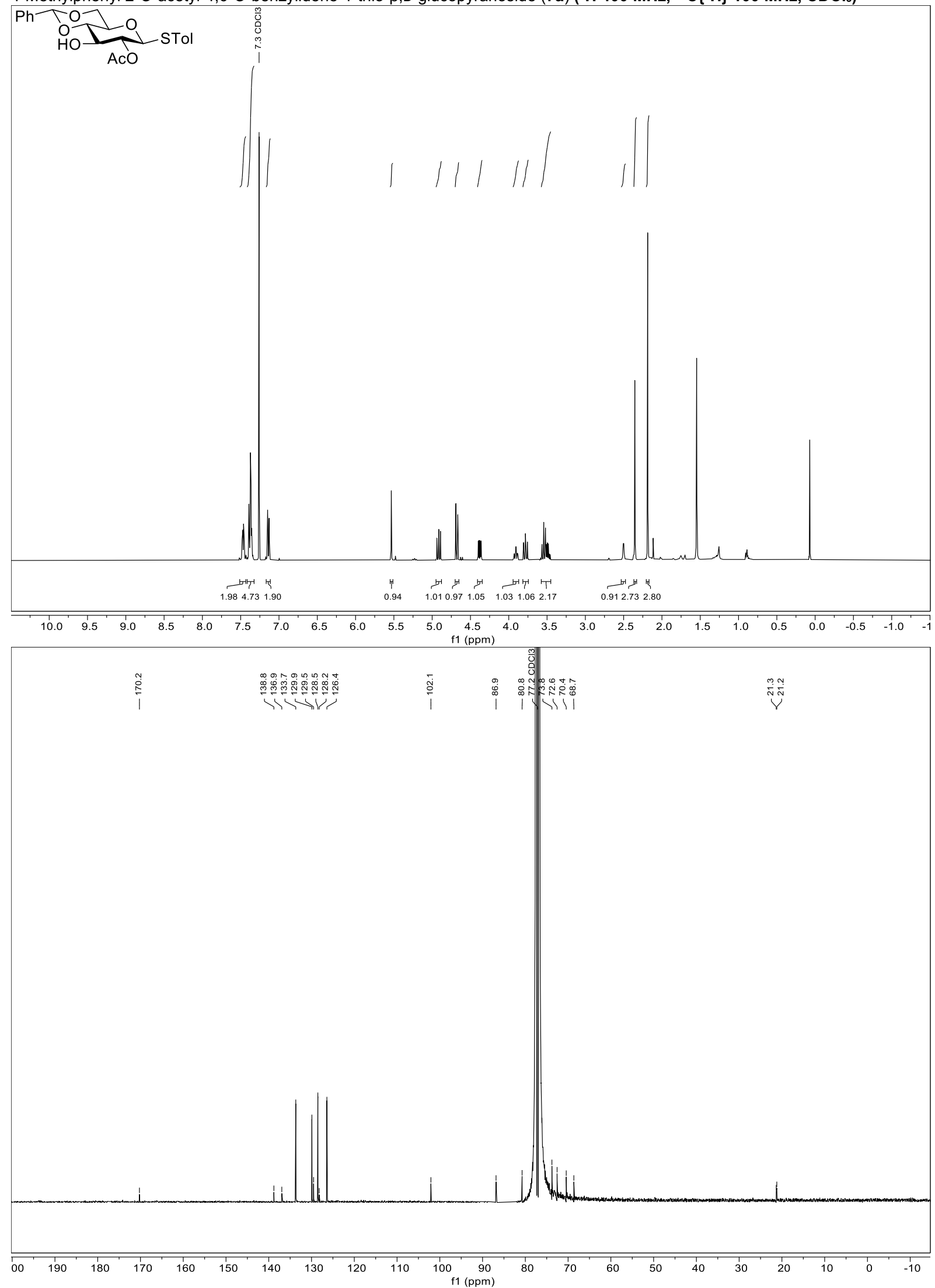
4-Methylphenyl 3-O-acetyl-4,6-O-benzylidene-1-thio- $\beta$,D-glucopyranoside $(7 \mathrm{~b})\left({ }^{1} \mathrm{H} 400 \mathrm{MHz},{ }^{13} \mathrm{C}\left\{{ }^{1} \mathrm{H}\right\} 100 \mathrm{MHz}, \mathrm{CDCl}_{3}\right)$
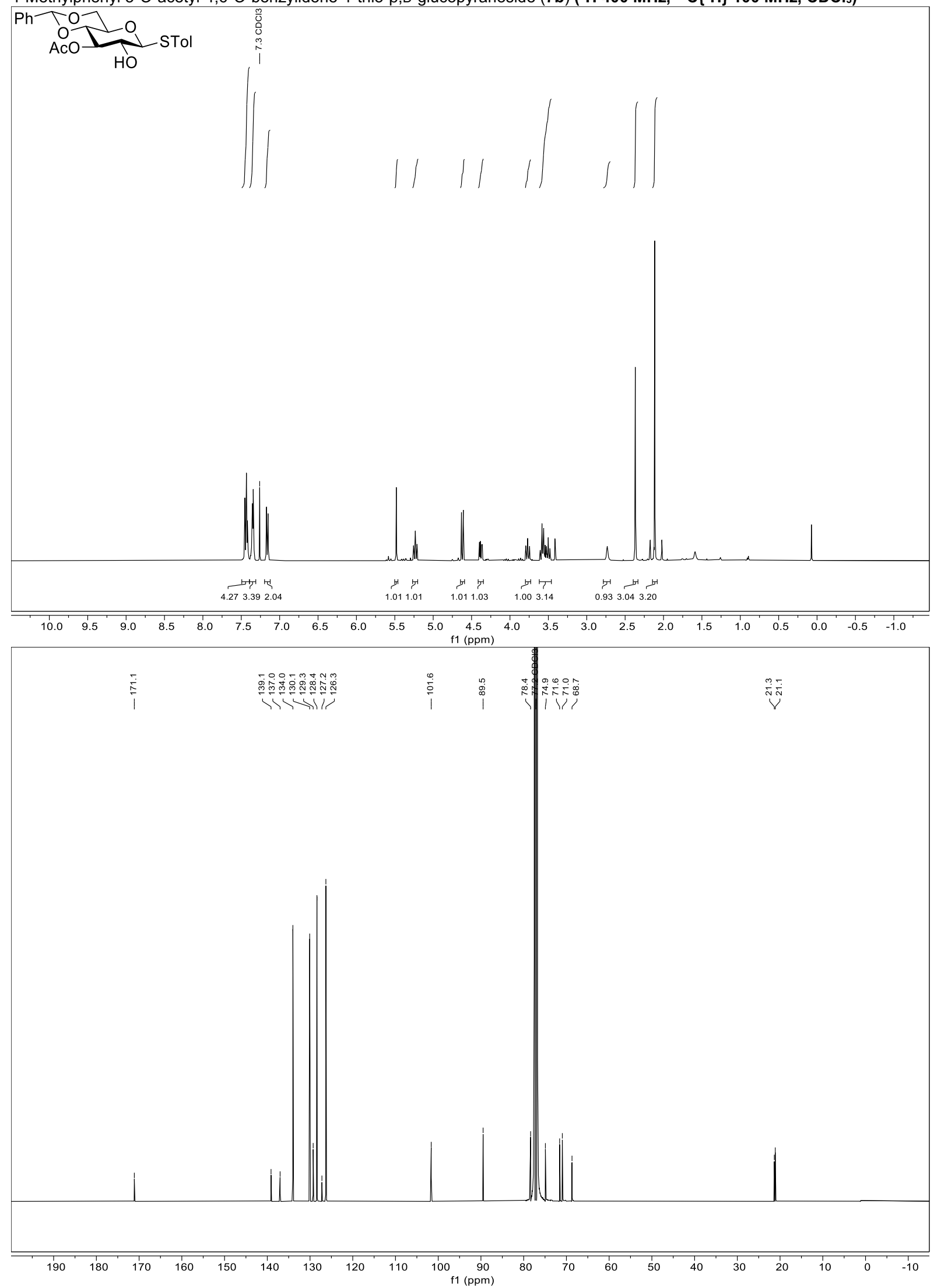
4-Methylphenyl 2,3-O-diacetyl-4,6-O-benzylidene-1-thio- $\beta, \mathrm{D}-$ glucopyranoside $(7 \mathrm{c})\left({ }^{1} \mathrm{H} 400 \mathrm{MHz},{ }^{13} \mathrm{C}\left\{{ }^{1} \mathrm{H}\right\} 100 \mathrm{MHz}, \mathrm{CDCl}_{3}\right)$
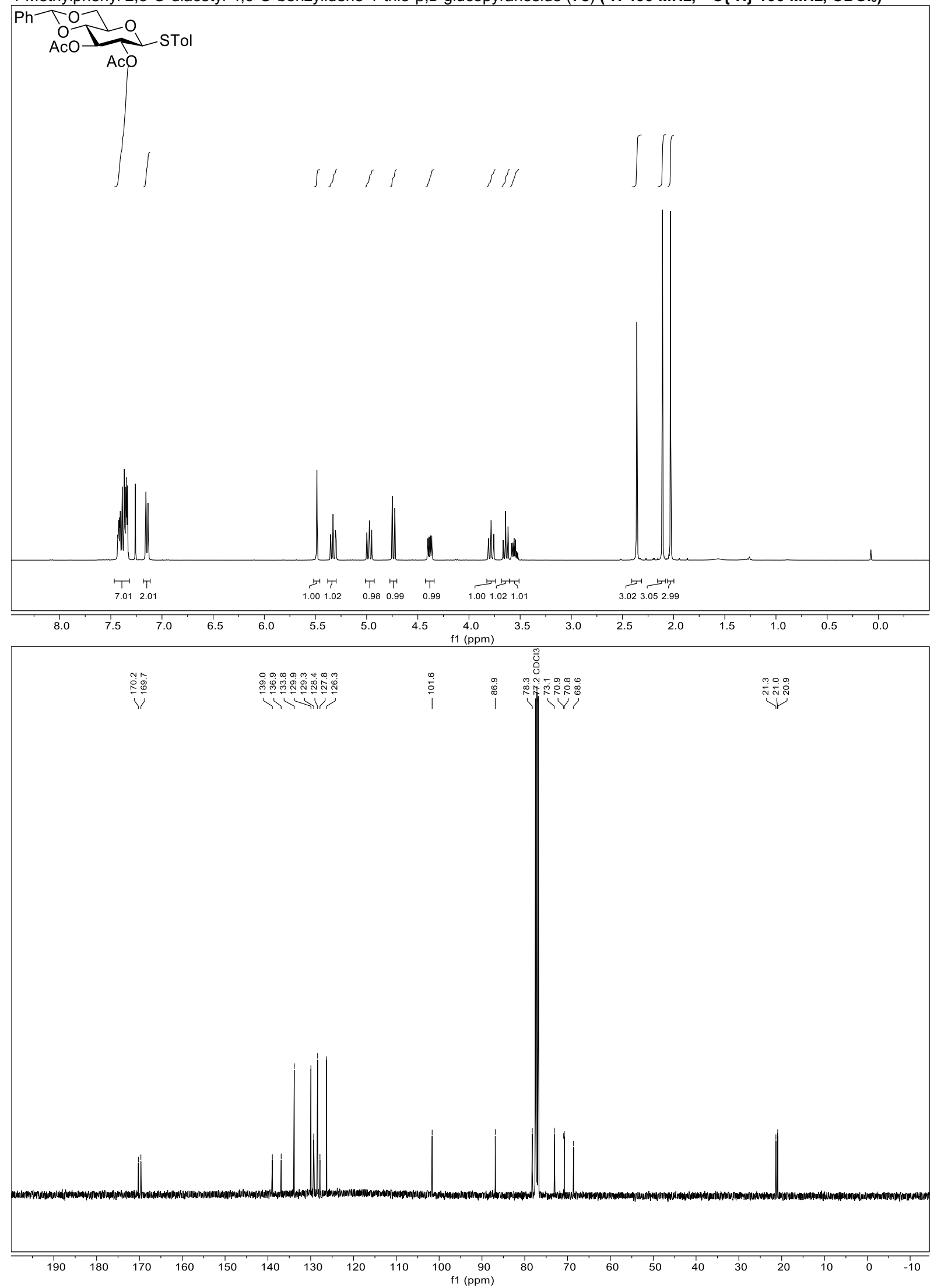
Methyl 4,6-O-benzylidene-2-O-isobutyryl-a,D-glucopyranoside (8a) $\left({ }^{1} \mathrm{H} 400 \mathrm{MHz},{ }^{13} \mathrm{C}\left\{{ }^{1} \mathrm{H}\right\} 100 \mathrm{MHz}, \mathrm{Acetone}-\mathrm{d}_{6}\right)$
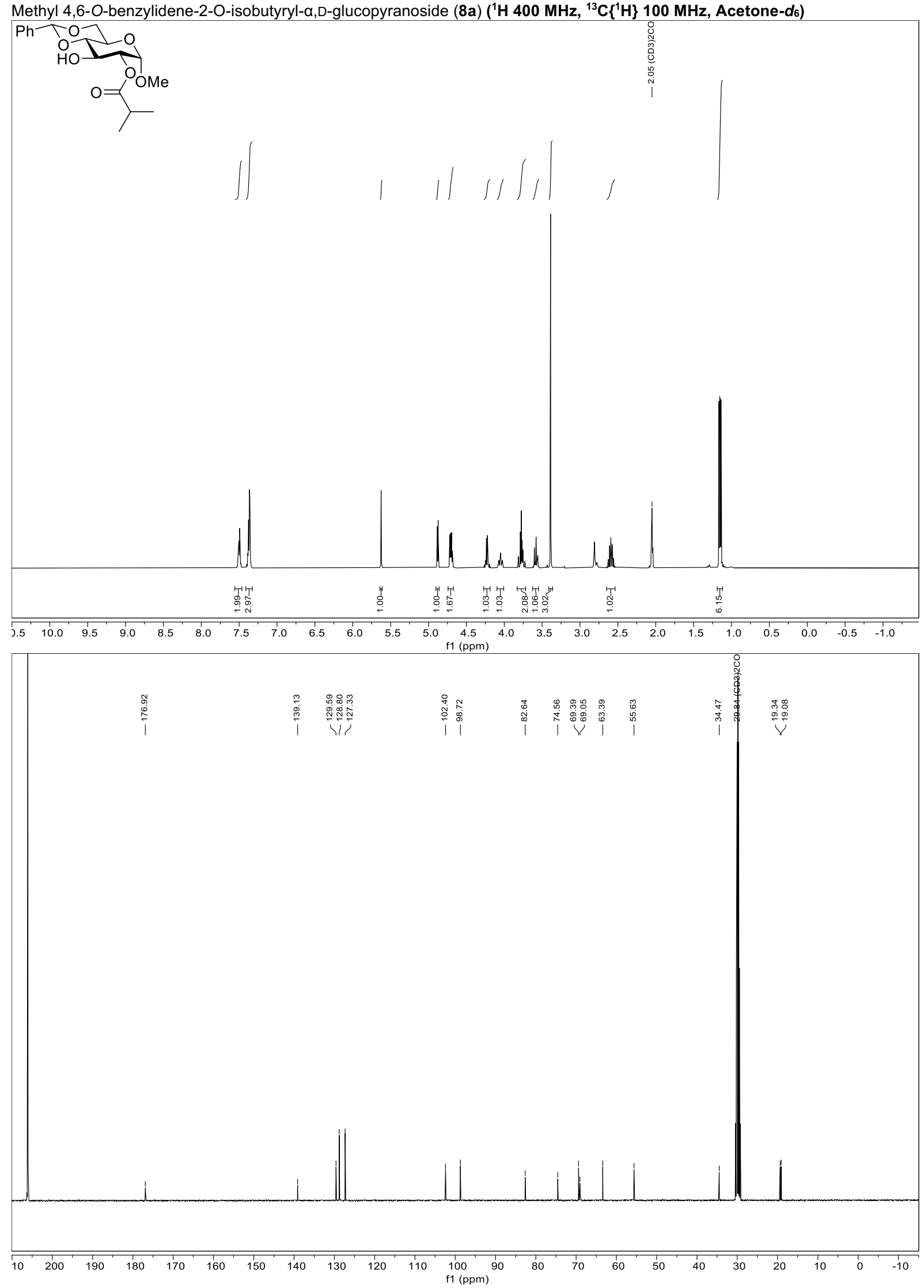
Methyl 4,6-O-benzylidene-3-O-isobutyryl-a,D-glucopyranoside (8b) $\left({ }^{1} \mathrm{H} 400 \mathrm{MHz},{ }^{13} \mathrm{C}\left\{{ }^{1} \mathrm{H}\right\} 100 \mathrm{MHz}\right.$, Acetone- $\left.d_{6}\right)$

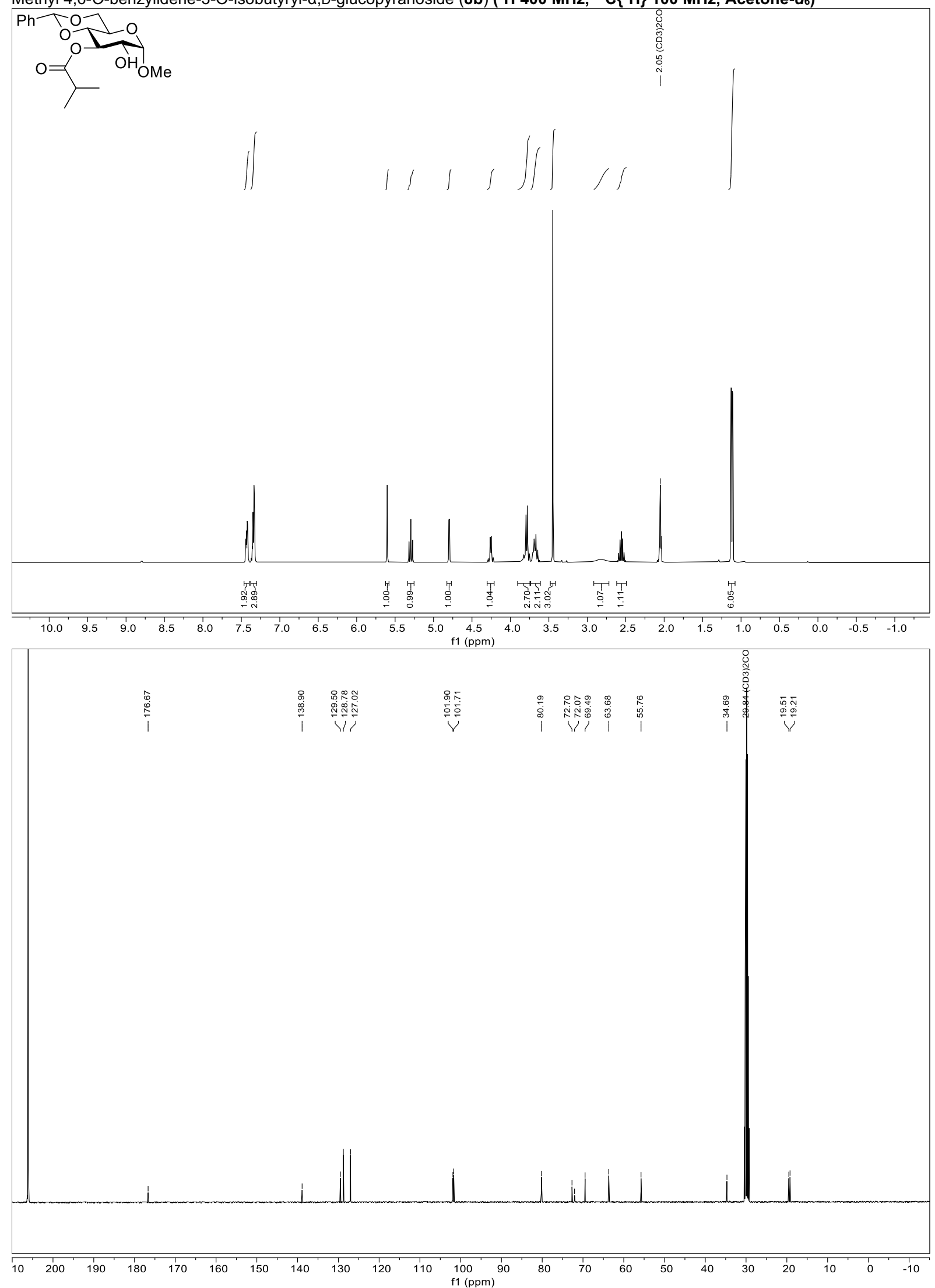


Methyl 4,6-O-benzylidene-2,3-O-diisobutyryl- $\alpha, \mathrm{D}-$ glucopyranoside $(8 \mathrm{c})\left({ }^{1} \mathrm{H} 400 \mathrm{MHz},{ }^{13} \mathrm{C}\left\{{ }^{1} \mathrm{H}\right\} 100 \mathrm{MHz}\right.$, Acetone- $\left.d_{6}\right)$
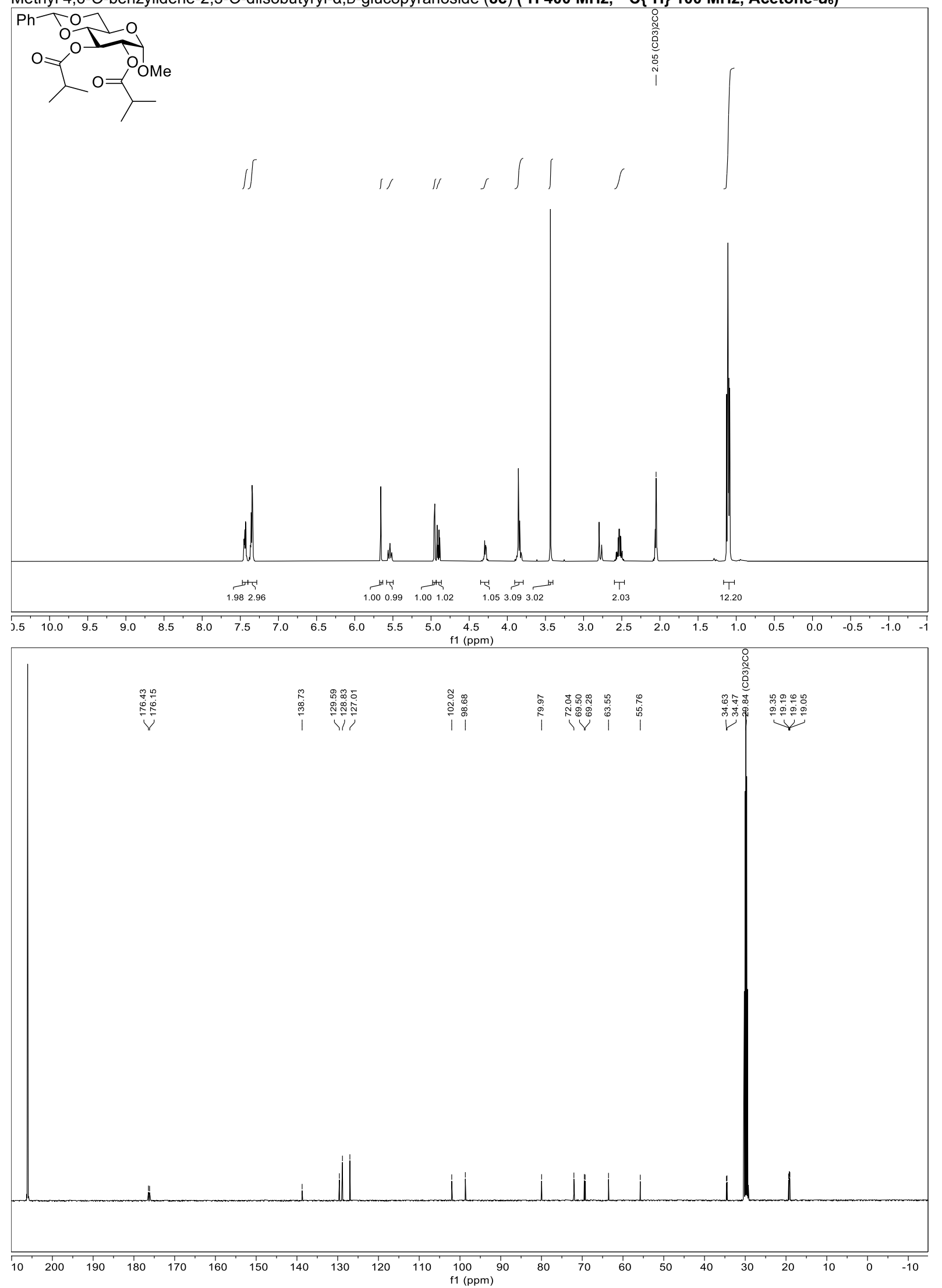
Methyl 4,6-O-methylidene-2-O-isobutyryl-a,D-glucopyranoside $(9 \mathrm{a})\left({ }^{1} \mathrm{H} 400 \mathrm{MHz},{ }^{13} \mathrm{C}\left\{{ }^{1} \mathrm{H}\right\} 100 \mathrm{MHz}, \mathrm{CDCl}_{3}\right)$

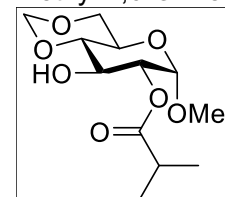

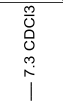
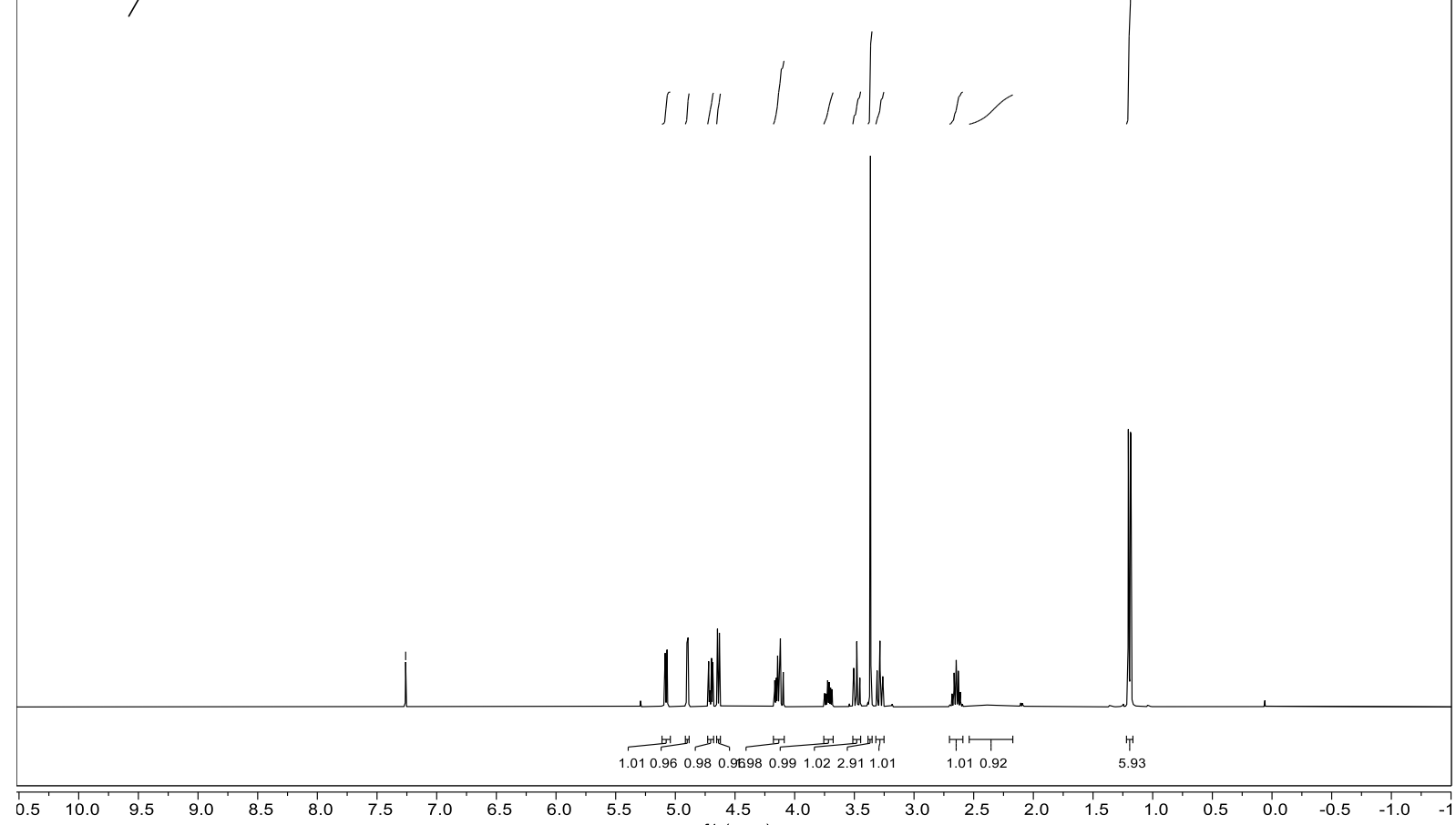
$\begin{array}{llllllllllllllllllllllllll}0.5 & 10.0 & 9.5 & 9.0 & 8.5 & 8.0 & 7.5 & 7.0 & 6.5 & 6.0 & 5.5 & 5.0 & \begin{array}{l}1.5 \\ \text { (ppm) }\end{array} & 4.0 & 3.5 & 3.0 & 2.5 & 2.0 & 1.5 & 1.0 & 0.5 & 0.0 & -0.5 & -1.0 & -1\end{array}$

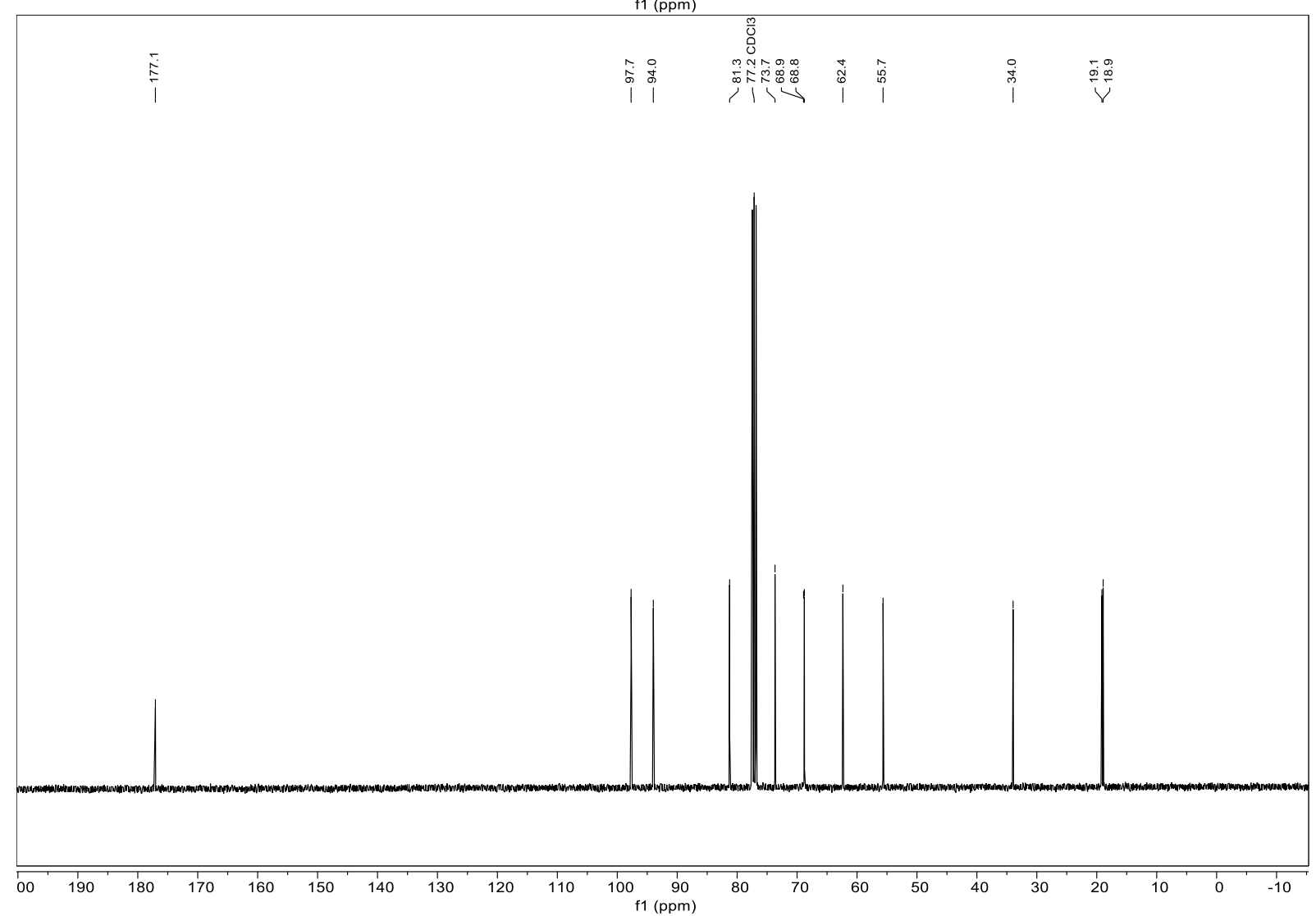


Methyl 4,6-O-methylidene-3-O-isobutyryl-a,D-glucopyranoside (9b) $\left({ }^{1} \mathrm{H} 400 \mathrm{MHz},{ }^{13} \mathrm{C}\left\{{ }^{1} \mathrm{H}\right\} 100 \mathrm{MHz}, \mathrm{CDCl}_{3}\right)$
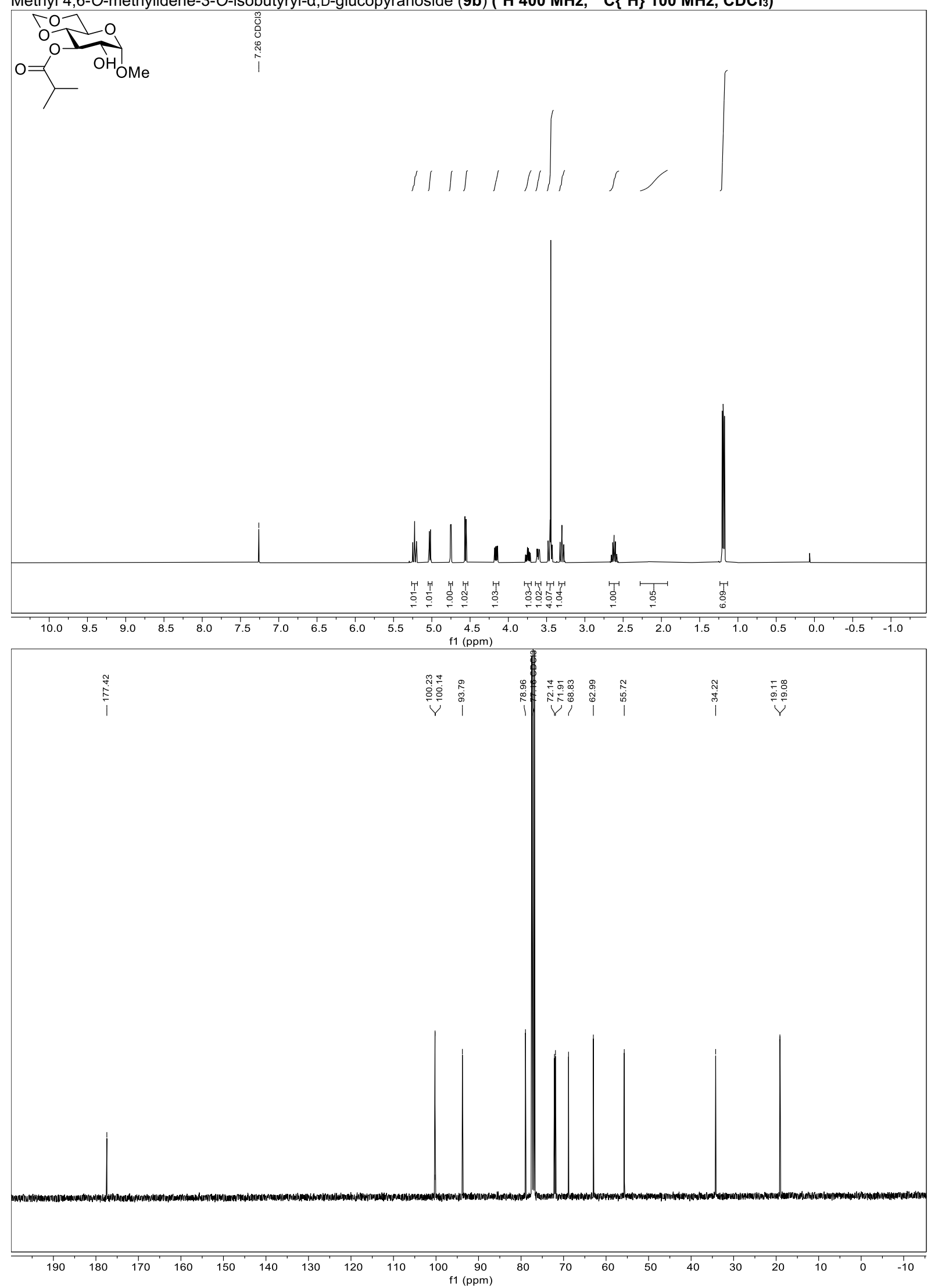
Methyl 4,6-O-methylidene-2,3-O-diisobutyryl- $\alpha, \mathrm{D}$-glucopyranoside (9c) $\left({ }^{1} \mathrm{H} 400 \mathrm{MHz},{ }^{13} \mathrm{C}\left\{{ }^{1} \mathrm{H}\right\} 100 \mathrm{MHz}, \mathrm{CDCl}_{3}\right)$

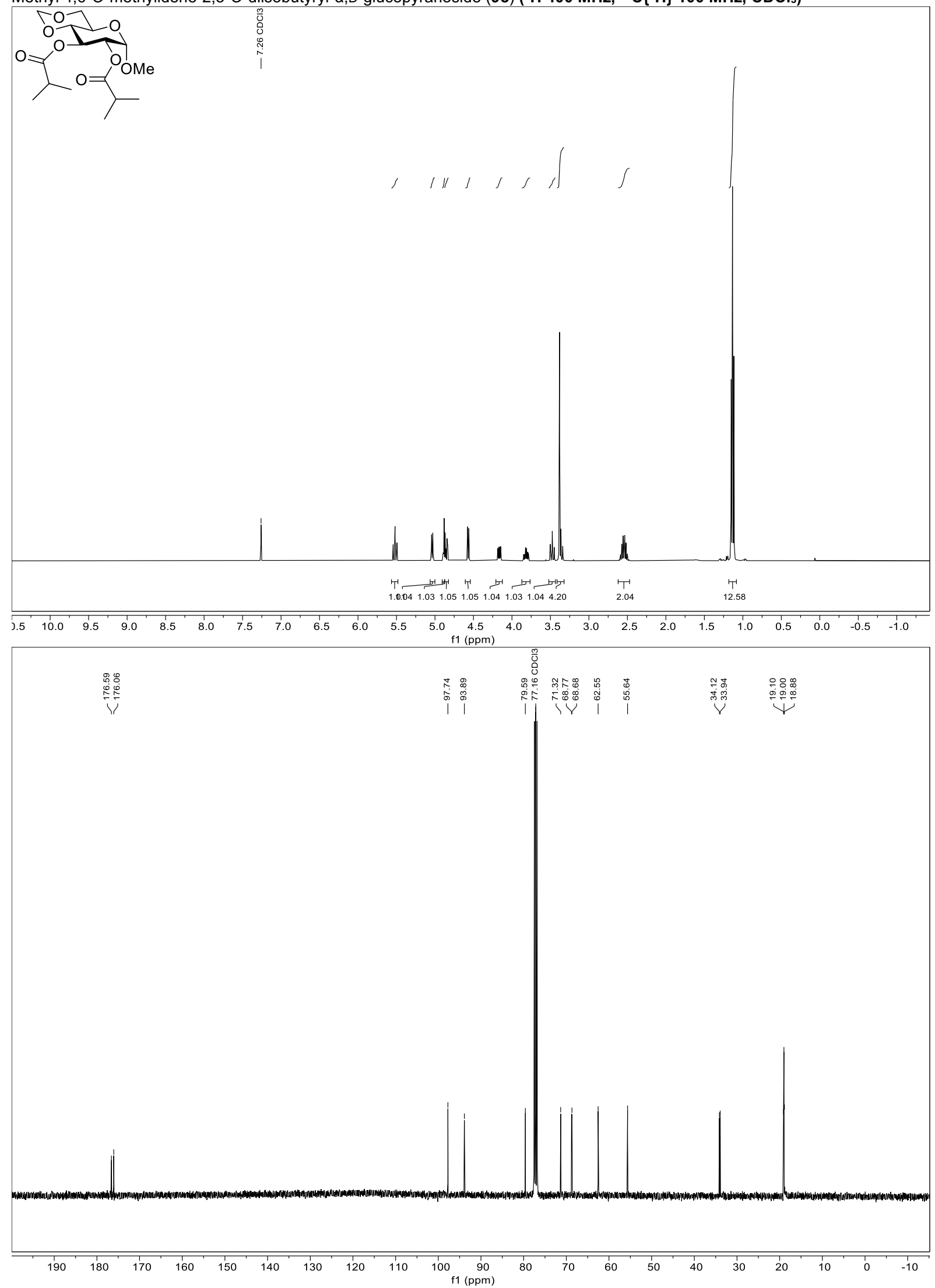


Methyl 2-O-acetyl-4,6-O-benzylidene- $\alpha, \mathrm{D}-$ mannopyranoside (10a) $\left({ }^{1} \mathrm{H} 400 \mathrm{MHz},{ }^{13} \mathrm{C}\left\{{ }^{1} \mathrm{H}\right\} 100 \mathrm{MHz}, \mathrm{MeOH}-\mathrm{d}_{4}\right)$

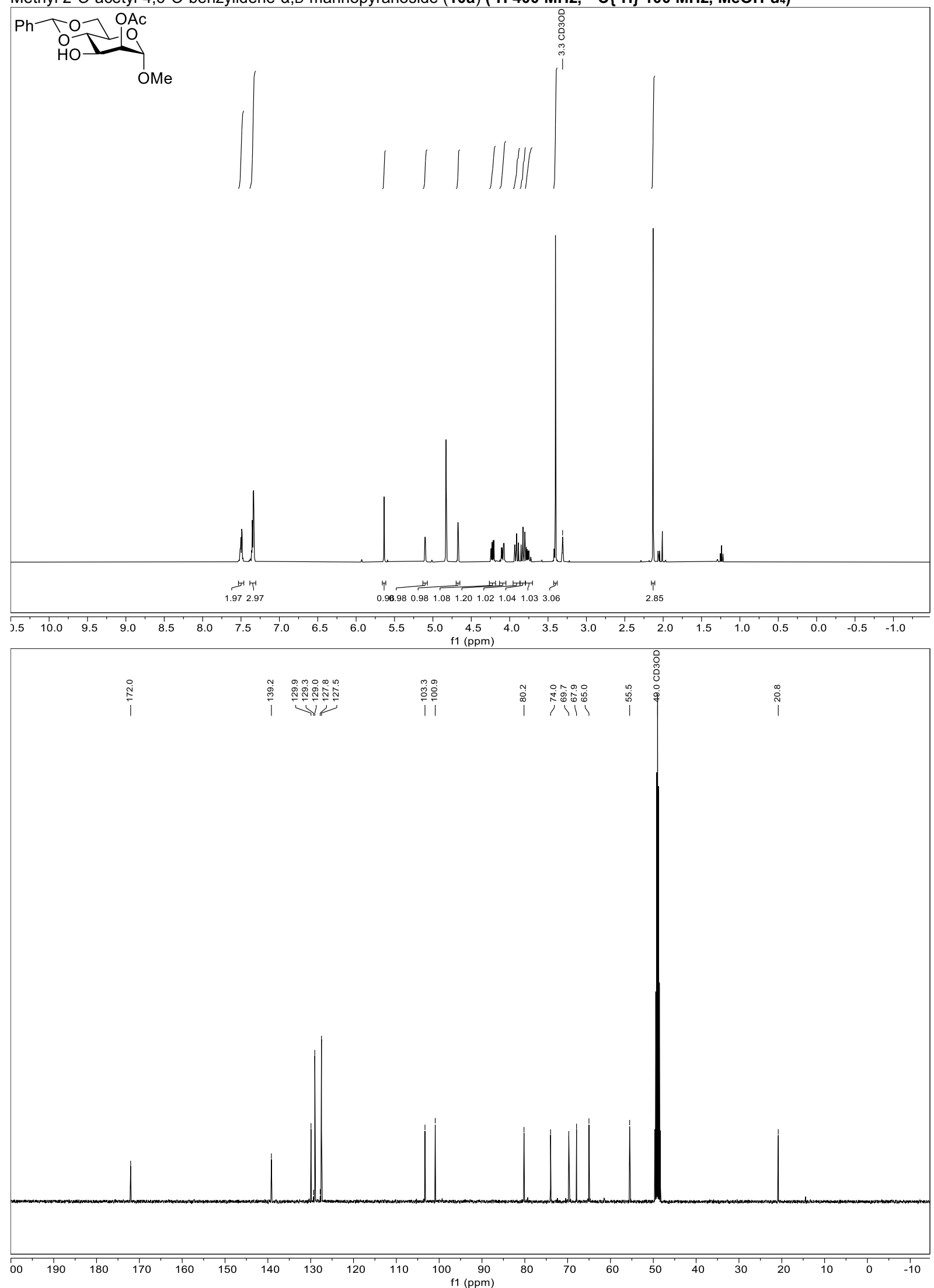


Methyl 3-O-acetyl-4,6-O-benzylidene-, ,D-mannopyranoside (10b) $\left({ }^{1} \mathrm{H} 400 \mathrm{MHz},{ }^{13} \mathrm{C}\left\{{ }^{1} \mathrm{H}\right\} 100 \mathrm{MHz}, \mathrm{MeOH}-\mathrm{d}_{4}\right)$

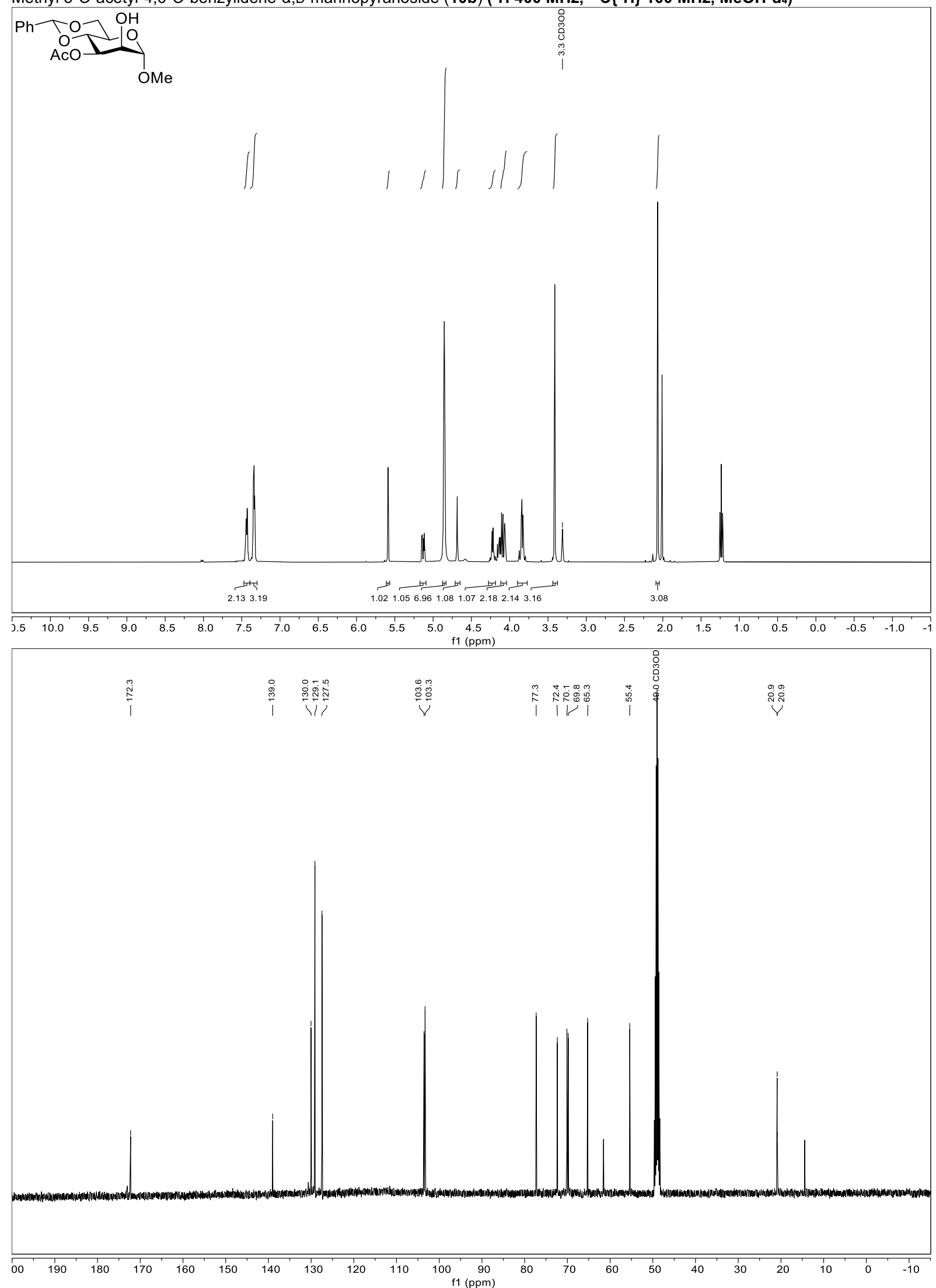


Methyl 2,3-O-diacetyl-4,6-O-benzylidene- $\alpha, \mathrm{D}-m a n n o p y r a n o s i d e ~(10 \mathrm{c})\left({ }^{1} \mathrm{H} 400 \mathrm{MHz},{ }^{13} \mathrm{C}\left\{{ }^{1} \mathrm{H}\right\} 100 \mathrm{MHz}, \mathrm{MeOH}-d_{4}\right)$

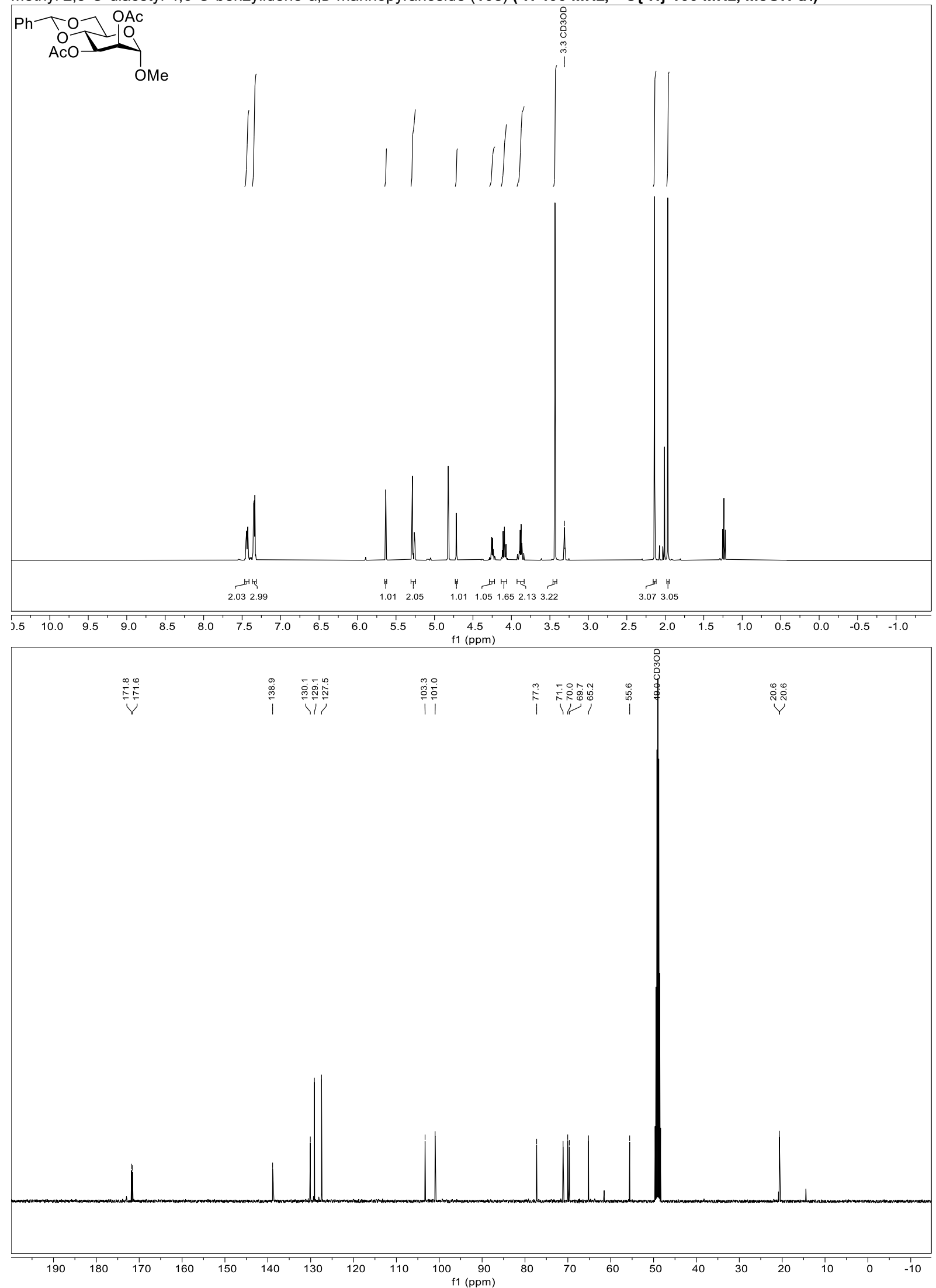


Methyl 2-O-acetyl-4,6-O-methylidene- $\alpha, \mathrm{D}-$ mannopyranoside (11a) $\left({ }^{1} \mathrm{H} 400 \mathrm{MHz},{ }^{13} \mathrm{C}\left\{{ }^{1} \mathrm{H}\right\} 100 \mathrm{MHz}, \mathrm{MeOH}-\mathrm{d}_{4}\right)$

$$
\text { OMe }
$$
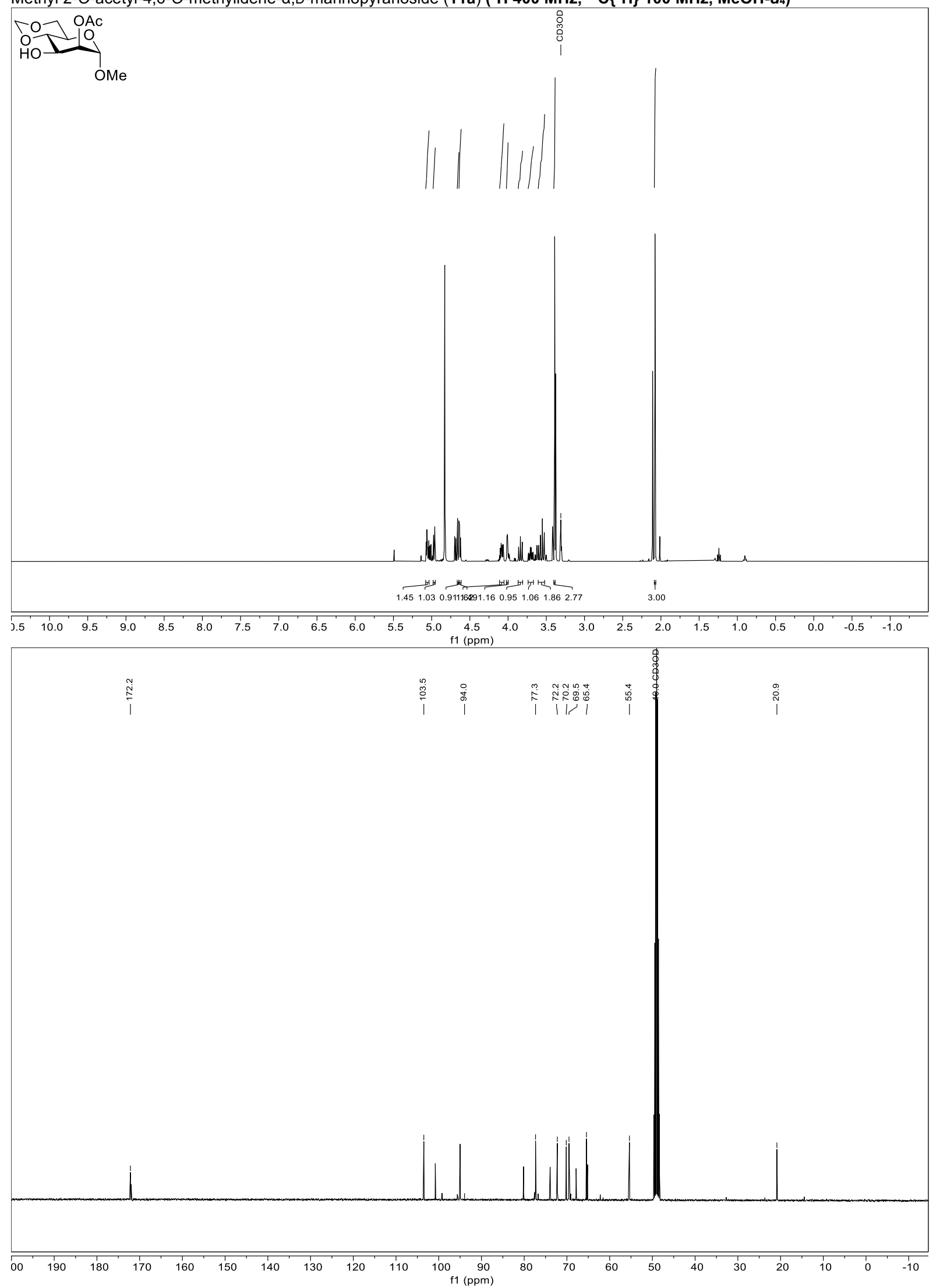
Methyl 3-O-acetyl-4,6-O-methylidene- $\alpha, \mathrm{D}-$ mannopyranoside $(11 \mathrm{~b})\left({ }^{1} \mathrm{H} 400 \mathrm{MHz},{ }^{13} \mathrm{C}\left\{{ }^{1} \mathrm{H}\right\} 100 \mathrm{MHz}, \mathrm{MeOH}-\mathrm{d}_{4}\right)$
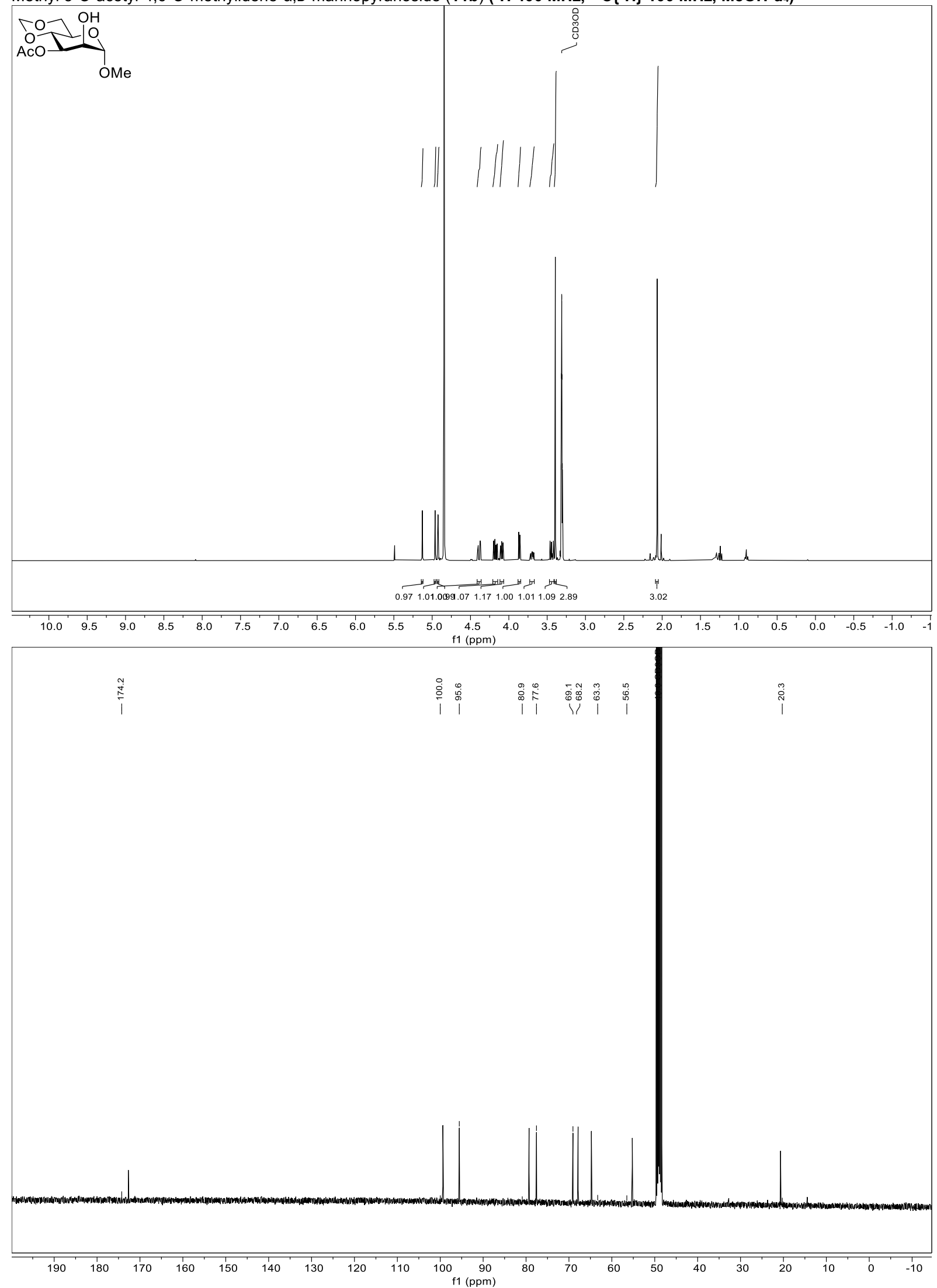
Methyl 2,3-O-diacetyl-4,6-O-methylidene-a,D-mannopyranoside (11c) $\left({ }^{1} \mathrm{H} 400 \mathrm{MHz},{ }^{13} \mathrm{C}\left\{{ }^{1} \mathrm{H}\right\} 100 \mathrm{MHz}, \mathrm{MeOH}-\mathrm{d}_{4}\right)$
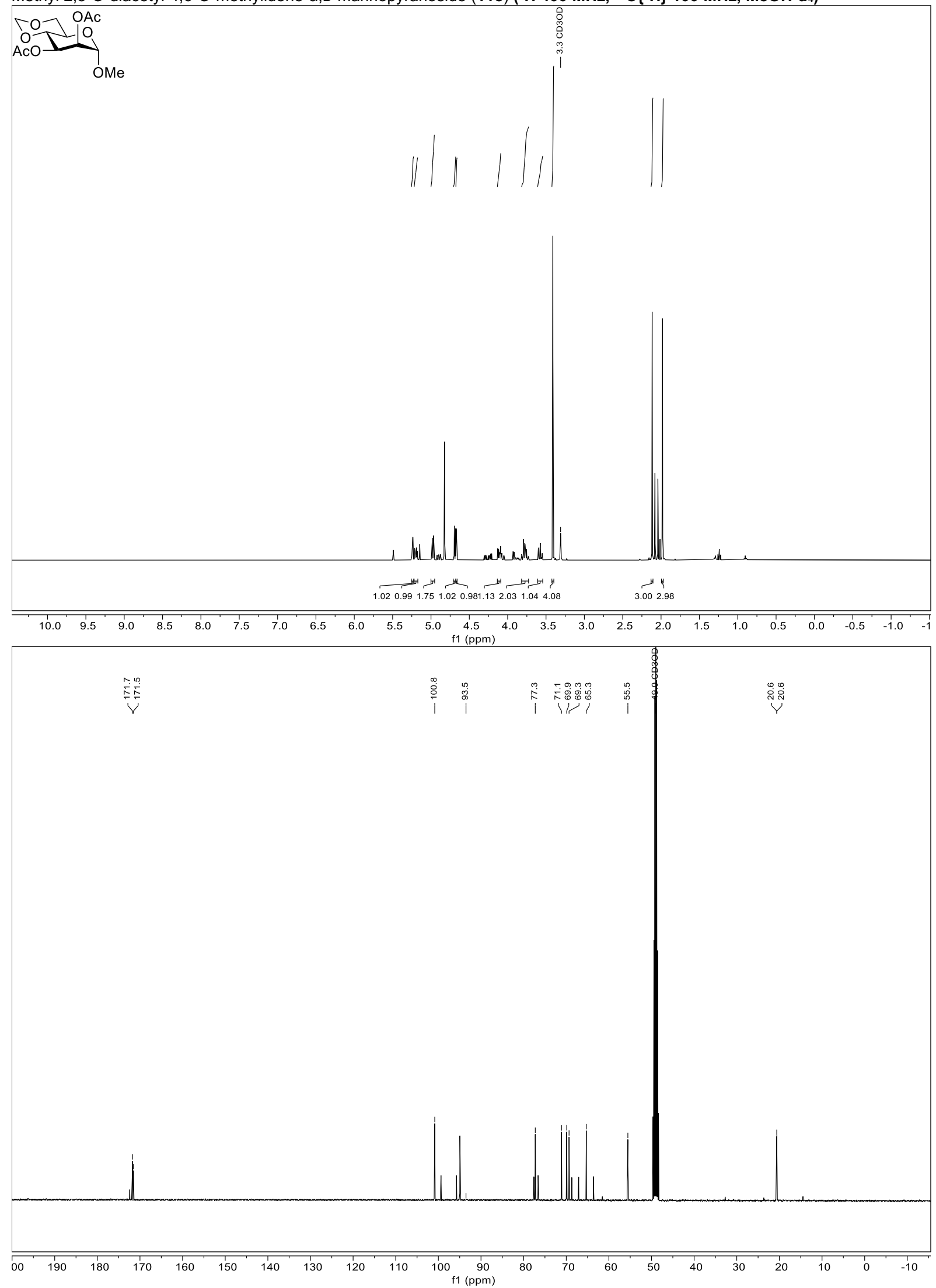
Methyl 2-O-acetyl-4,6-O-(2-naphtylidene)- $\alpha, \mathrm{D}-$ mannopyranoside (12a) $\left({ }^{1} \mathrm{H} 400 \mathrm{MHz},{ }^{13} \mathrm{C}\left\{{ }^{1} \mathrm{H}\right\} 100 \mathrm{MHz}, \mathrm{MeOH}-\mathrm{d}_{4}\right)$
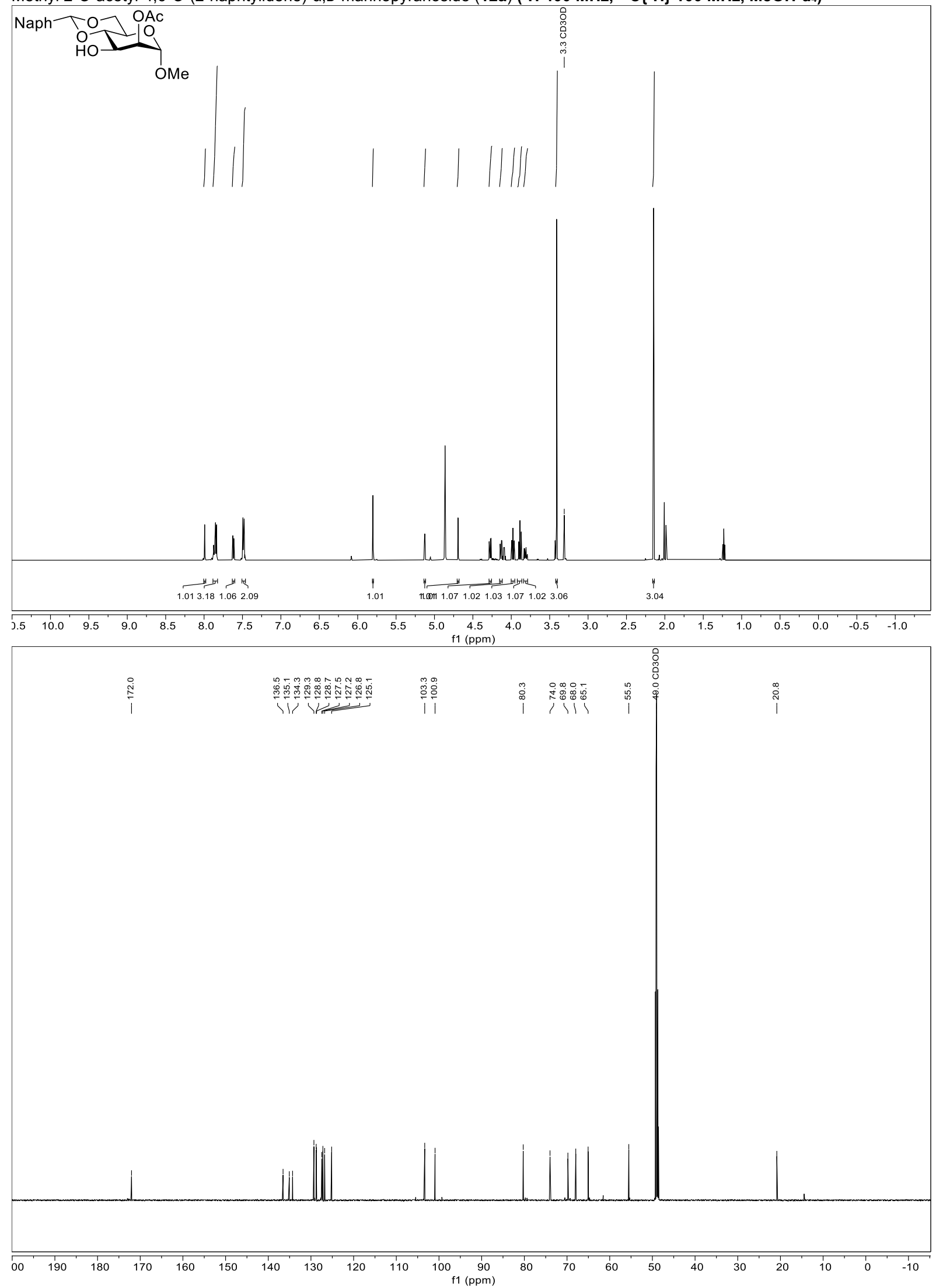
Methyl 3-O-acetyl-4,6-O-(2-naphtylidene)-a,D-mannopyranoside (12b) $\left({ }^{1} \mathrm{H} 400 \mathrm{MHz},{ }^{13} \mathrm{C}\left\{{ }^{1} \mathrm{H}\right\} 100 \mathrm{MHz}, \mathrm{MeOH}-\mathrm{d}_{4}\right)$
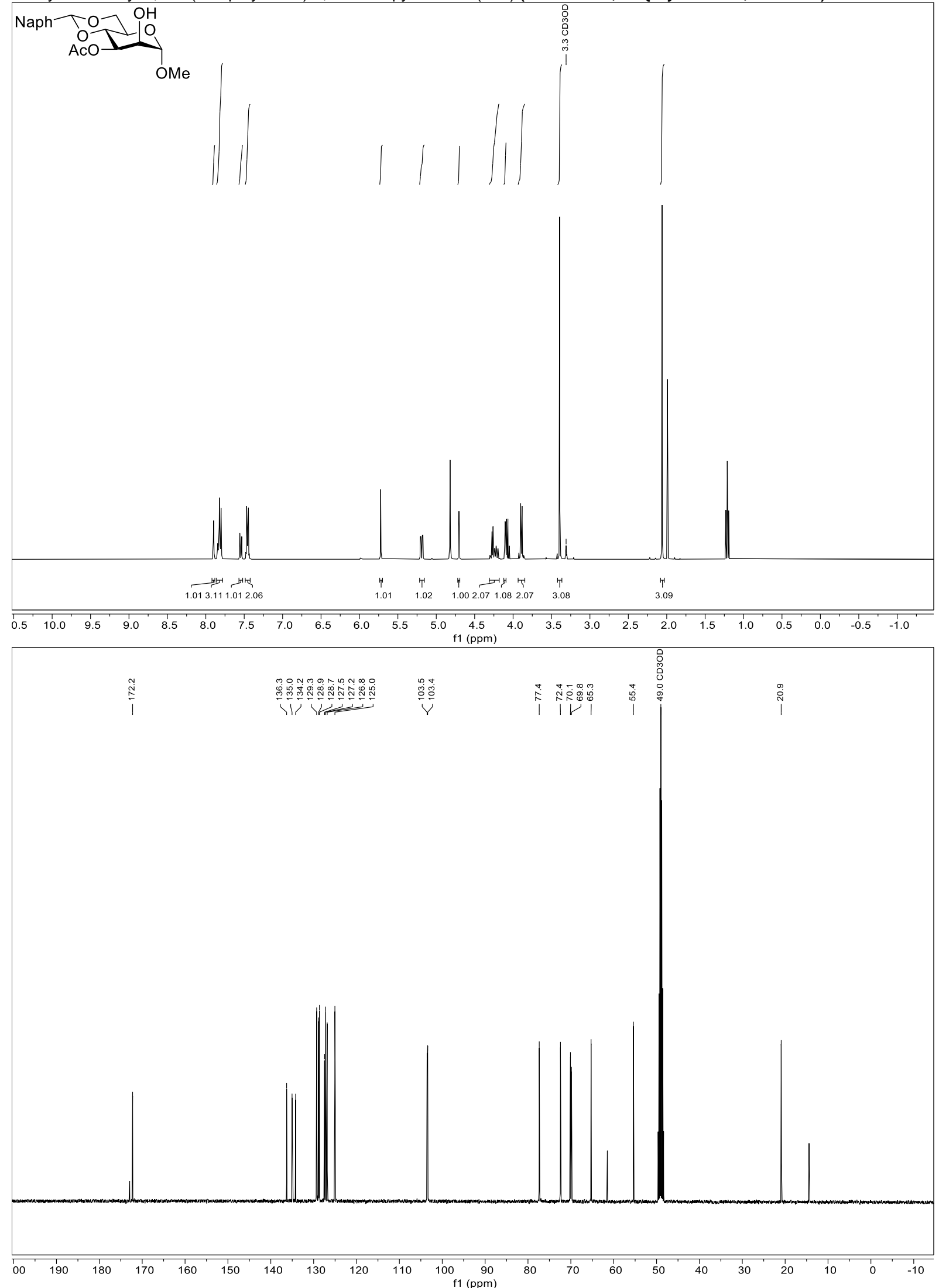
Methyl 2,3-O-diacetyl-4,6-O-(2-naphtylidene)-a,D-mannopyranoside (12c) $\left({ }^{1} \mathrm{H} 400 \mathrm{MHz},{ }^{13} \mathrm{C}\left\{{ }^{1} \mathrm{H}\right\} 100 \mathrm{MHz}, \mathrm{MeOH}-\mathrm{d}_{4}\right)$
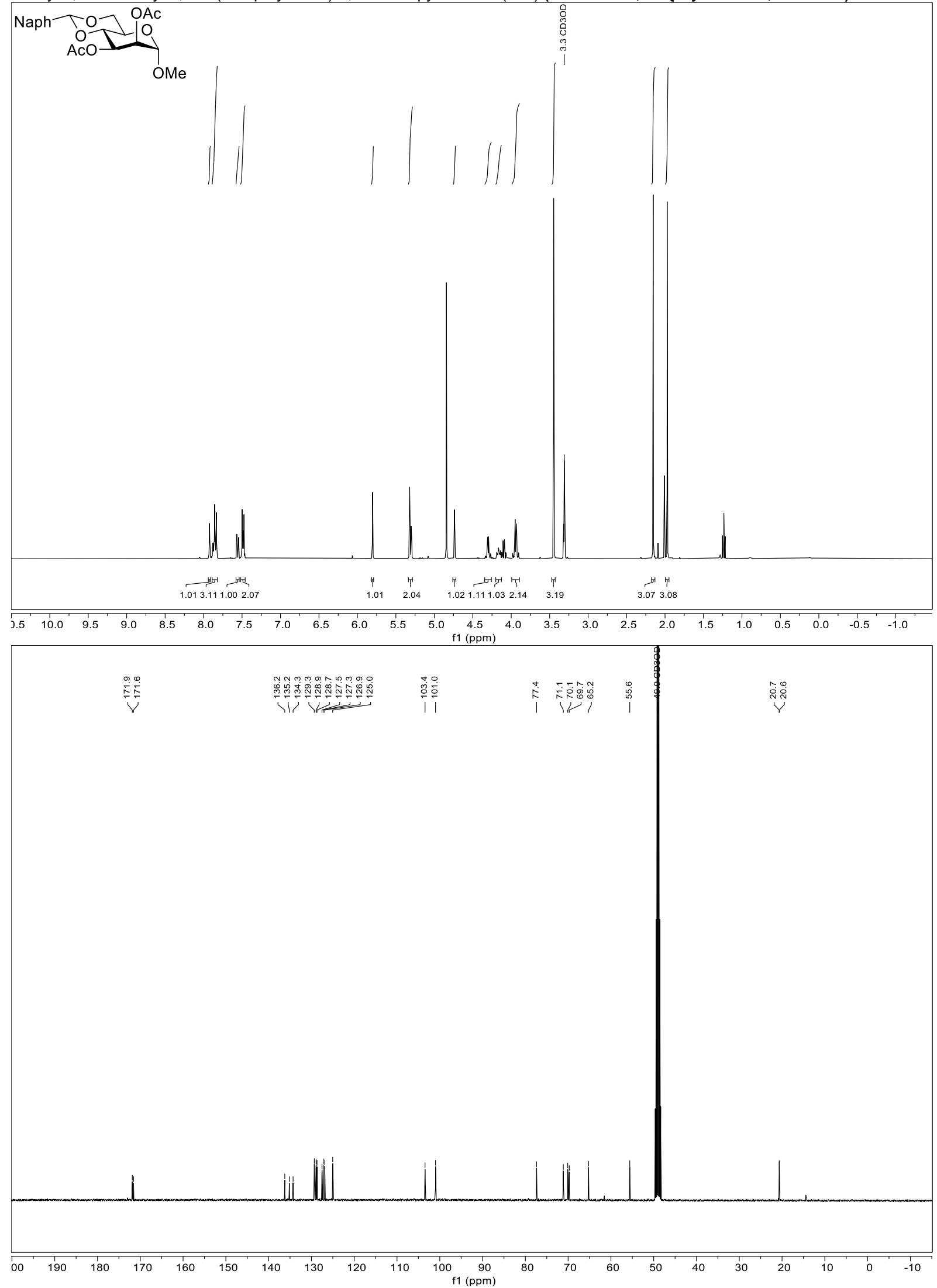
Methyl 2-O-acetyl-4,6-O-benzylidene- $\alpha, \mathrm{D}-$ galactopyranoside (13a) $\left({ }^{1} \mathrm{H} 400 \mathrm{MHz},{ }^{13} \mathrm{C}\left\{{ }^{1} \mathrm{H}\right\} 100 \mathrm{MHz}, \mathrm{CDCl}_{3}\right)$
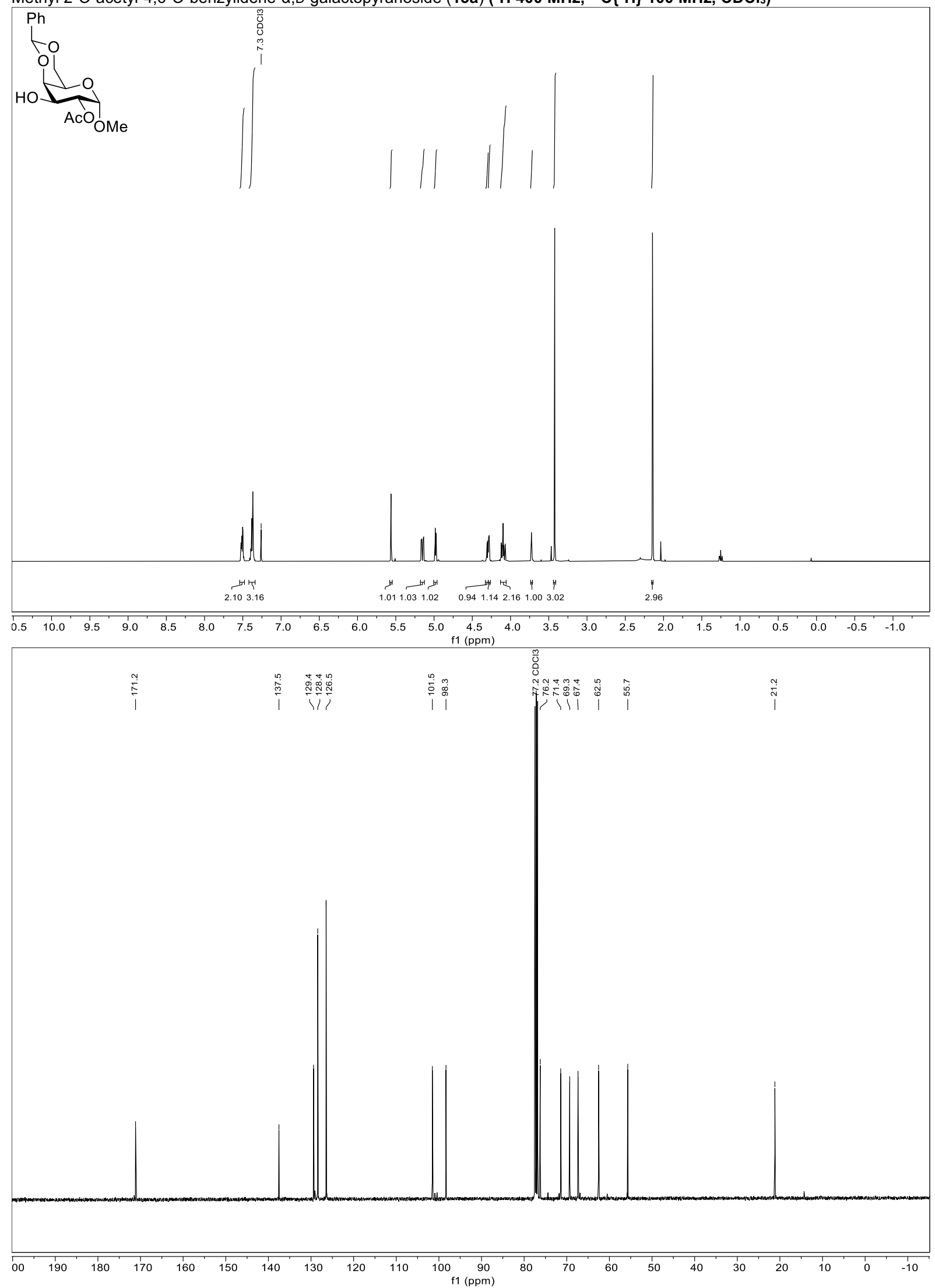
Methyl 3-O-acetyl-4,6-O-benzylidene-, $\mathrm{D}$-galactopyranoside (13b) $\left({ }^{1} \mathrm{H} 400 \mathrm{MHz},{ }^{13} \mathrm{C}\left\{{ }^{1} \mathrm{H}\right\} 100 \mathrm{MHz}, \mathrm{CDCl}_{3}\right)$

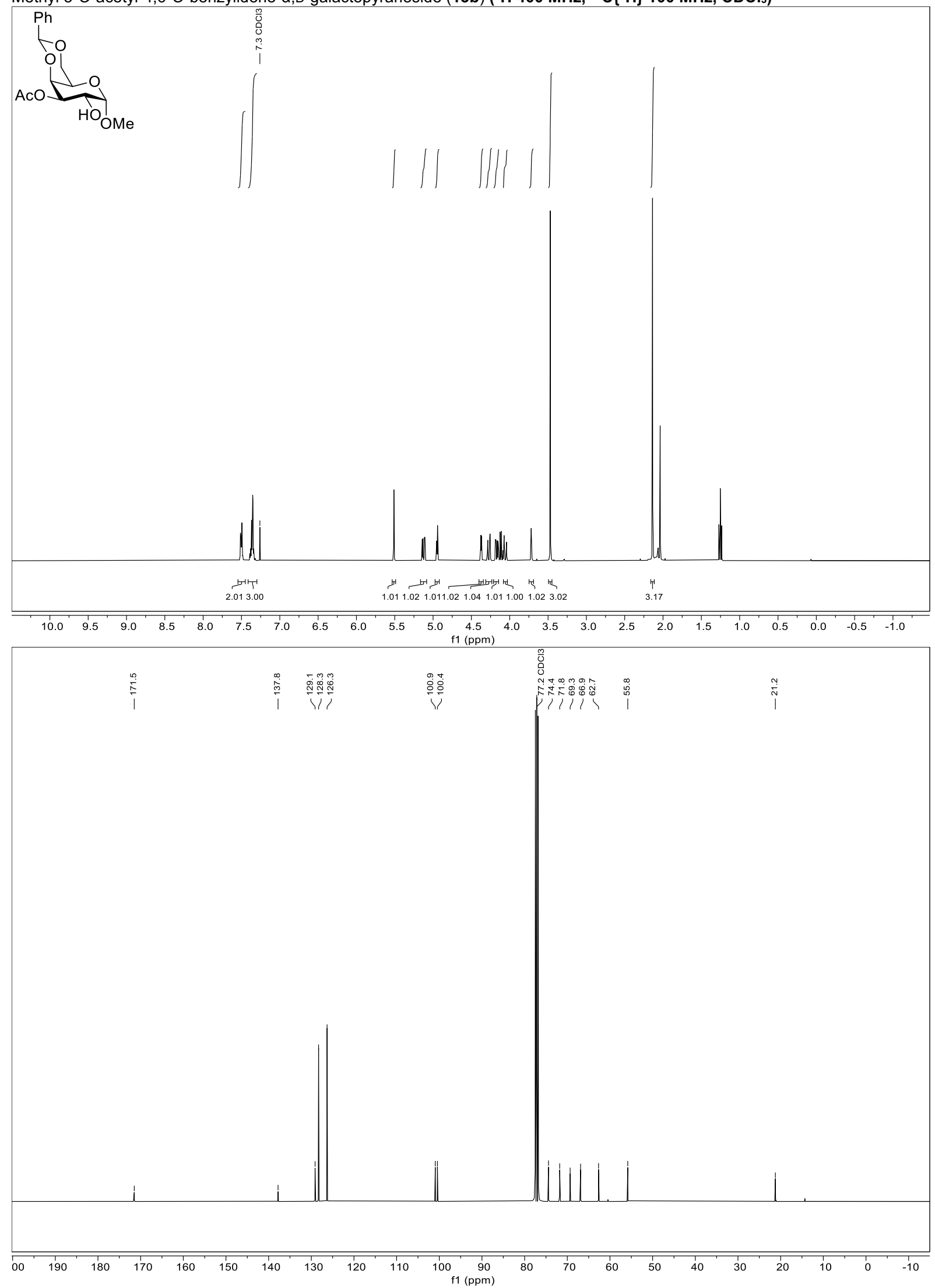


Methyl 2,3-O-diacetyl-4,6-O-benzylidene- $\alpha$,D-galactopyranoside $(13 \mathrm{C})\left({ }^{1} \mathrm{H} 400 \mathrm{MHz},{ }^{13} \mathrm{C}\left\{{ }^{1} \mathrm{H}\right\} 100 \mathrm{MHz}, \mathrm{CDCl}_{3}\right)$

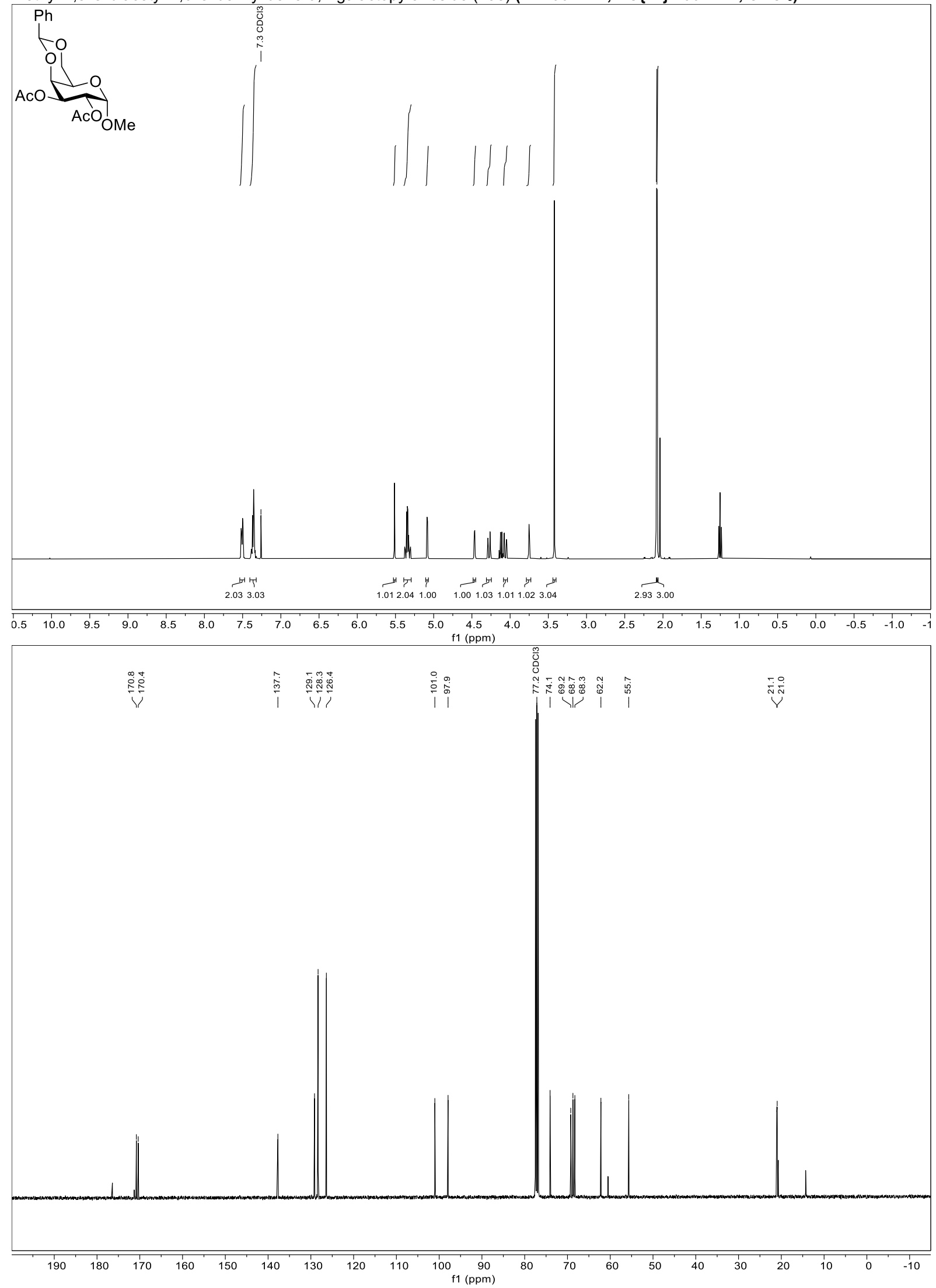


Methyl 2-O-isobutyryl-4,6-O-benzylidene- $\alpha, \mathrm{D}-m a n n o p y r a n o s i d e(18 \mathrm{a})\left({ }^{1} \mathrm{H} 400 \mathrm{MHz},{ }^{13} \mathrm{C}\left\{{ }^{1} \mathrm{H}\right\} 100 \mathrm{MHz}, \mathrm{MeOH}-\mathrm{d}_{4}\right)$
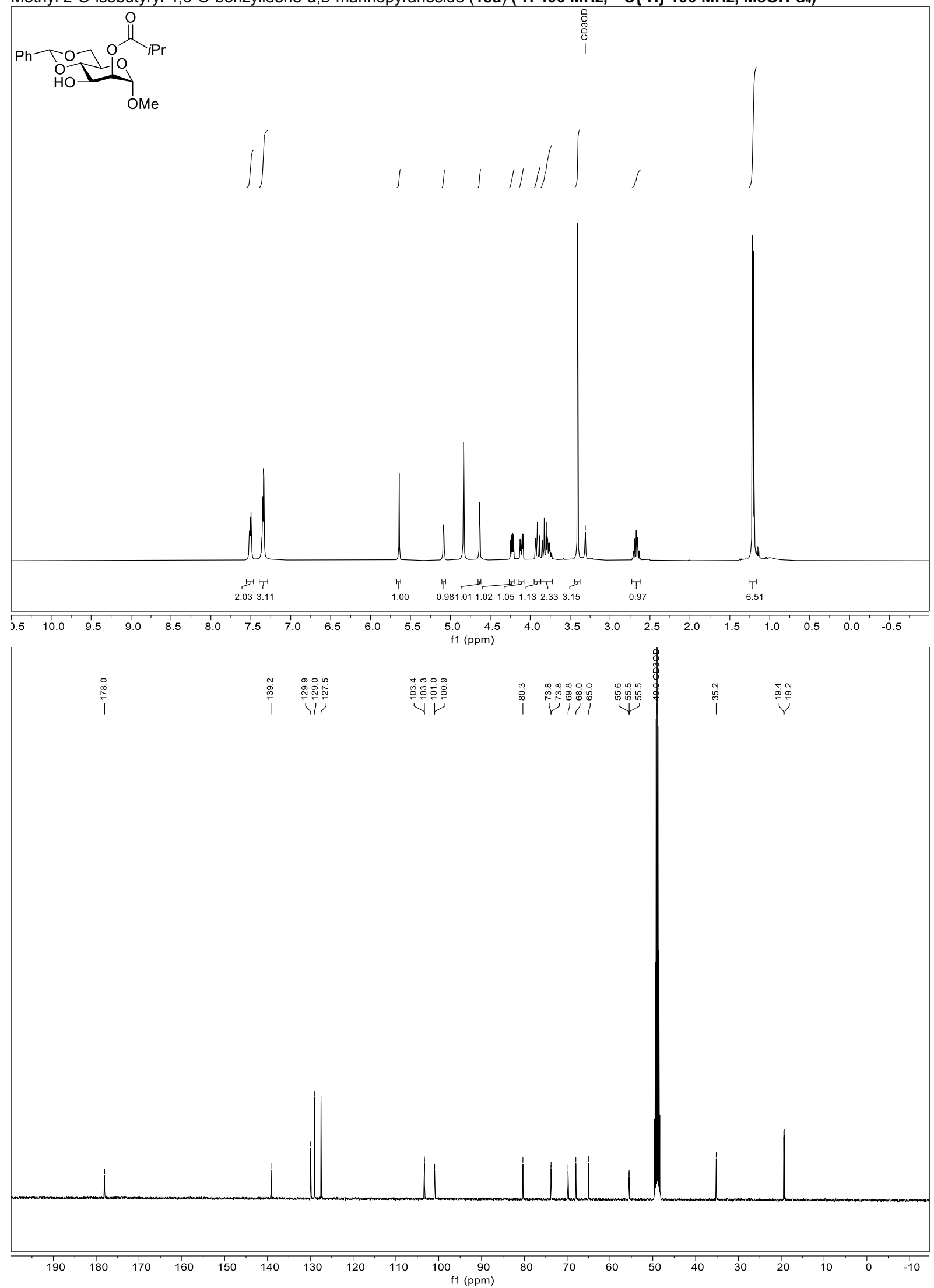
Methyl 3-O-isobutyryl-4,6-O-benzylidene-a,D-mannopyranoside (18b) $\left({ }^{1} \mathrm{H} 400 \mathrm{MHz},{ }^{13} \mathrm{C}\left\{{ }^{1} \mathrm{H}\right\} 100 \mathrm{MHz}, \mathrm{MeOH}-d_{4}\right)$
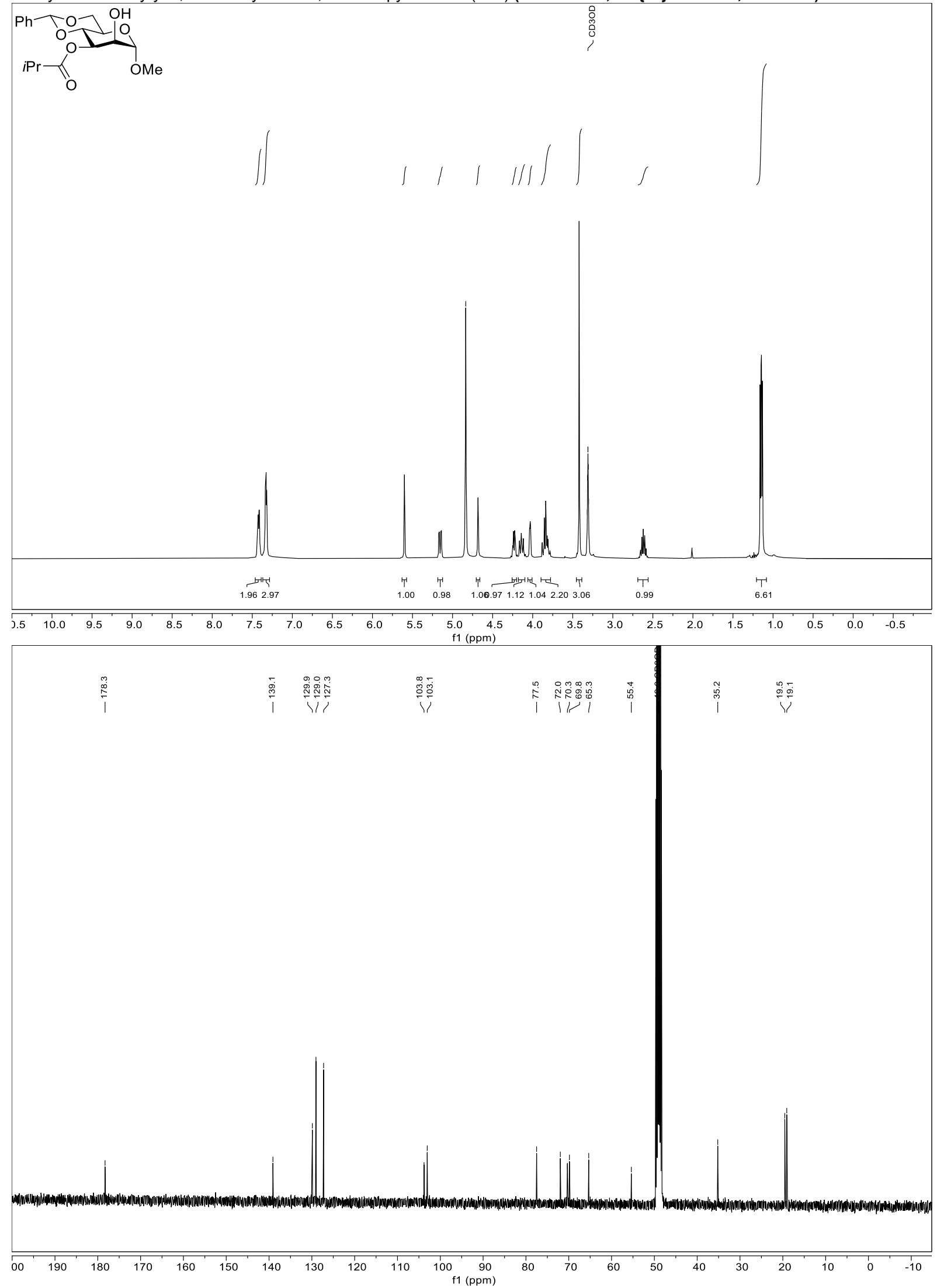
Methyl 2,3-O-diisobutyryl-4,6-O-benzylidene-a,D-mannopyranoside (18c) $\left({ }^{1} \mathrm{H} 400 \mathrm{MHz},{ }^{13} \mathrm{C}\left\{{ }^{1} \mathrm{H}\right\} 100 \mathrm{MHz}, \mathrm{MeOH}-\mathrm{d}_{4}\right)$
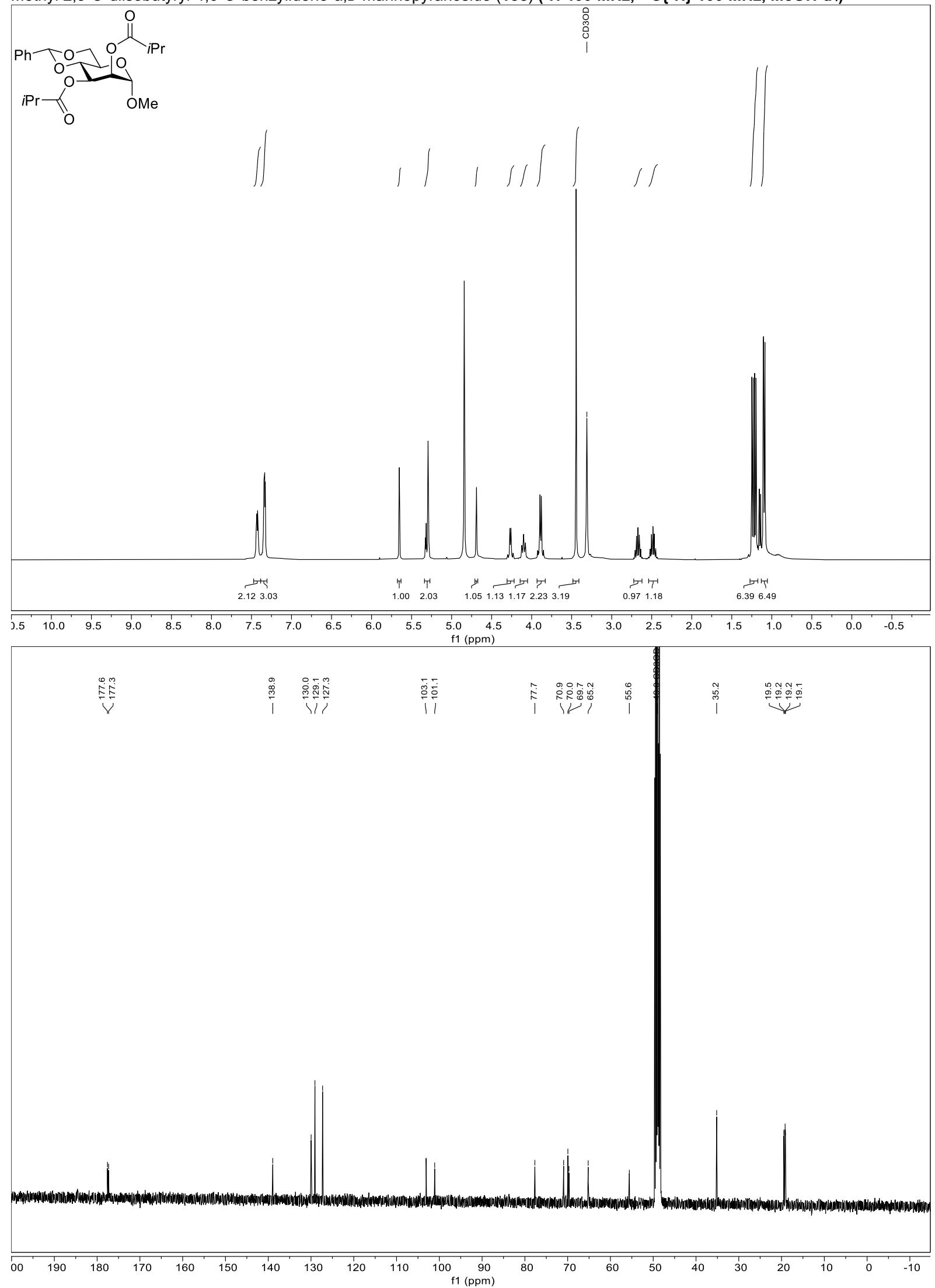


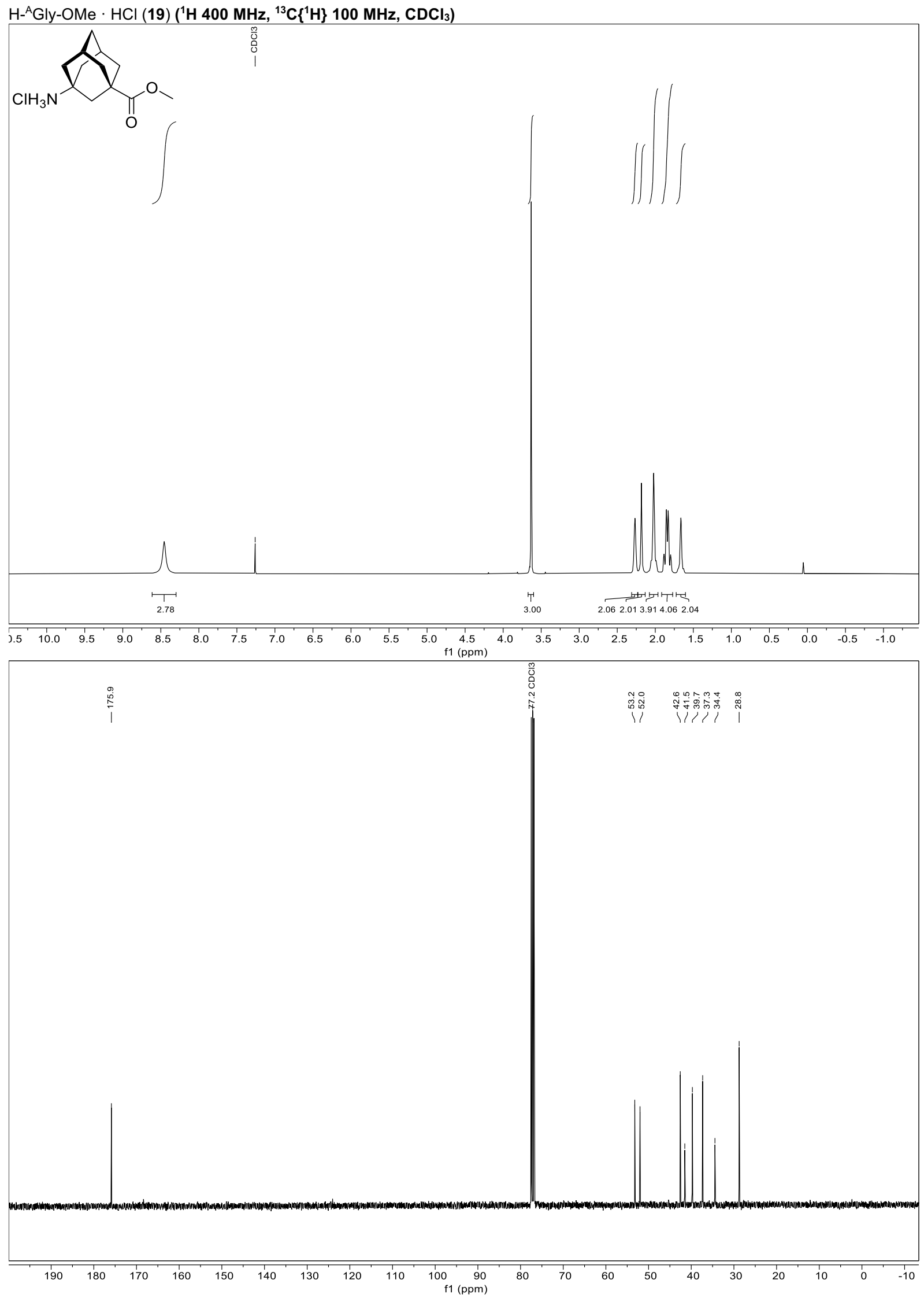

S69 
Boc-D-Pmh- ${ }^{\mathrm{A} G l y-L-V a l-L-P h e-O M e ~(2) ~}\left({ }^{1} \mathrm{H} 400 \mathrm{MHz},{ }^{13} \mathrm{C}\left\{{ }^{1} \mathrm{H}\right\} 100 \mathrm{MHz}, \mathrm{CDCl}_{3}\right)$
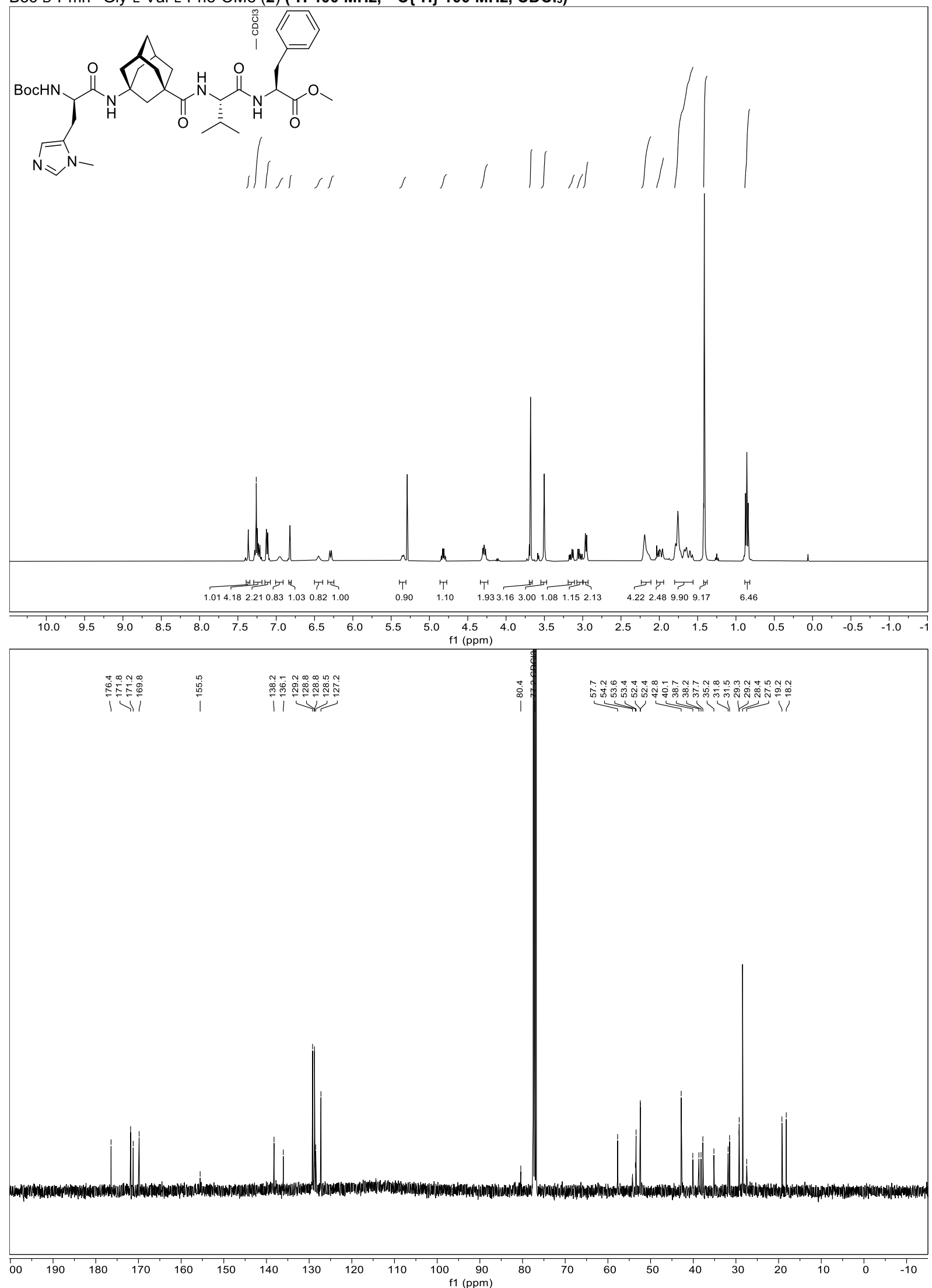

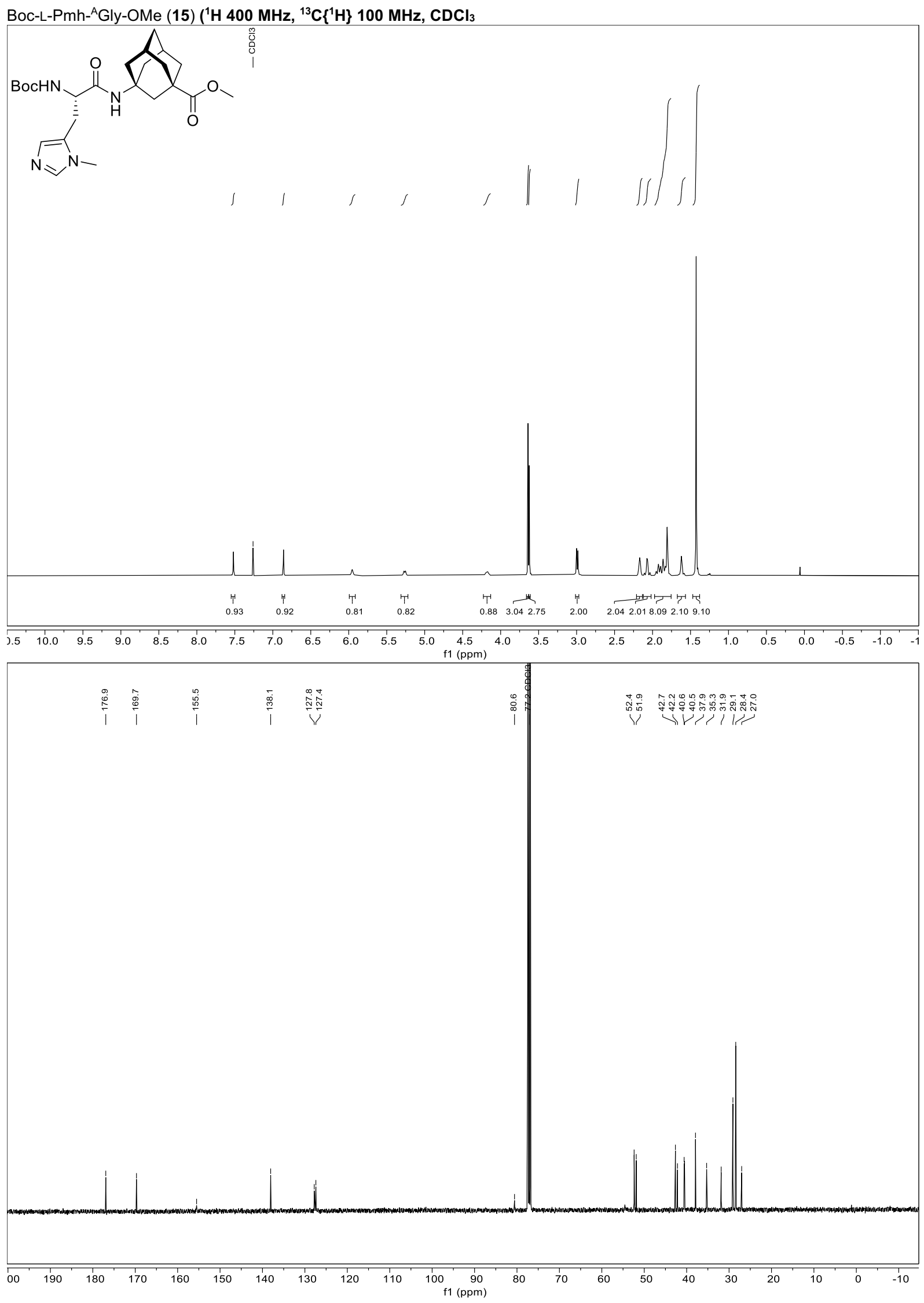

S71 
Boc-L-Pmh-A Gly-L-Cha-OMe (16) $\left({ }^{1} \mathrm{H} 400 \mathrm{MHz},{ }^{13} \mathrm{C}\left\{{ }^{1} \mathrm{H}\right\} 100 \mathrm{MHz}, \mathrm{CDCl}_{3}\right)$
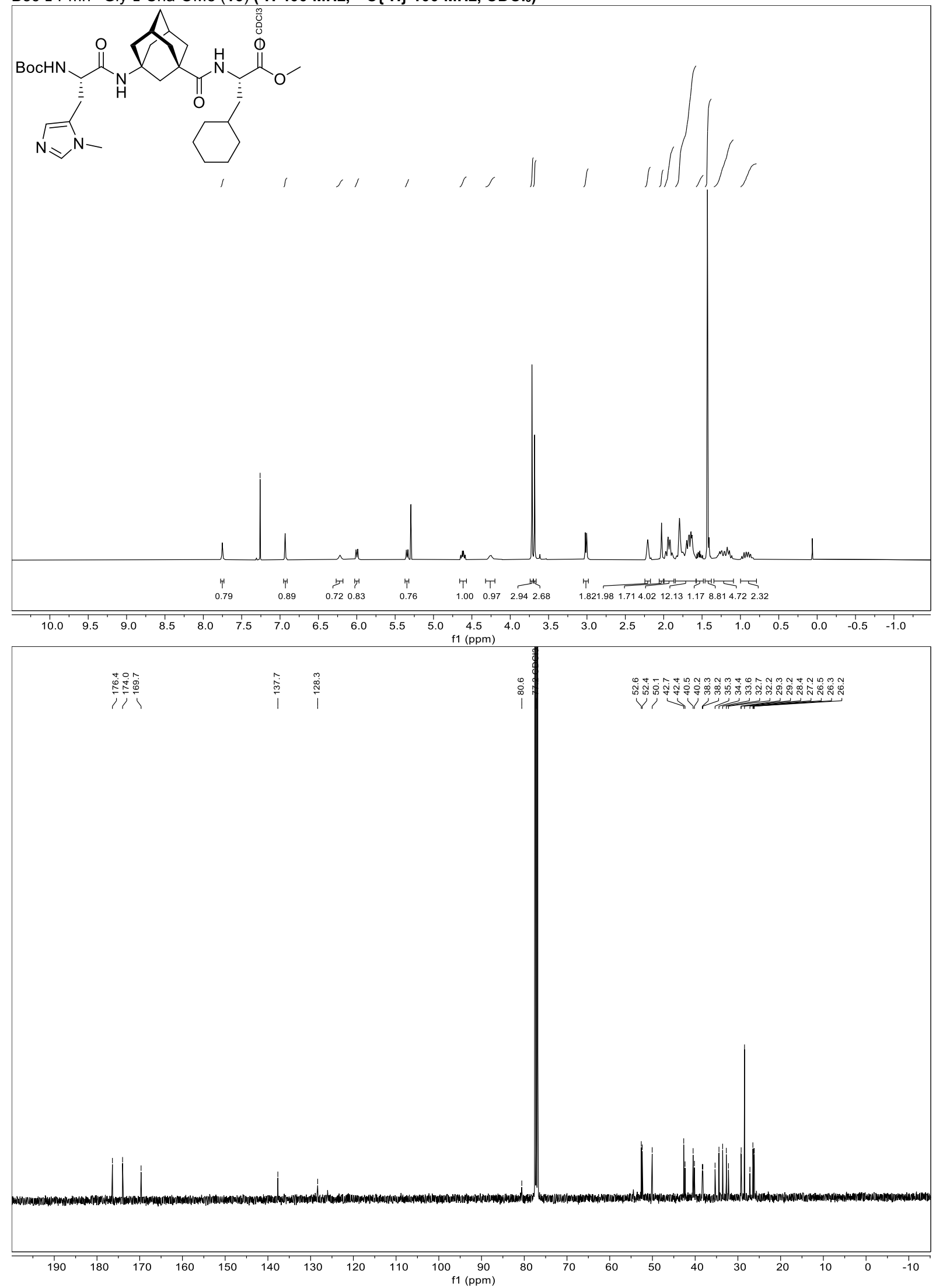
Boc-D-Pmh-OMe (20) ( $\left.{ }^{1} \mathrm{H} 400 \mathrm{MHz},{ }^{13} \mathrm{C}\left\{{ }^{1} \mathrm{H}\right\} 100 \mathrm{MHz}, \mathrm{CDCl}_{3}\right)$

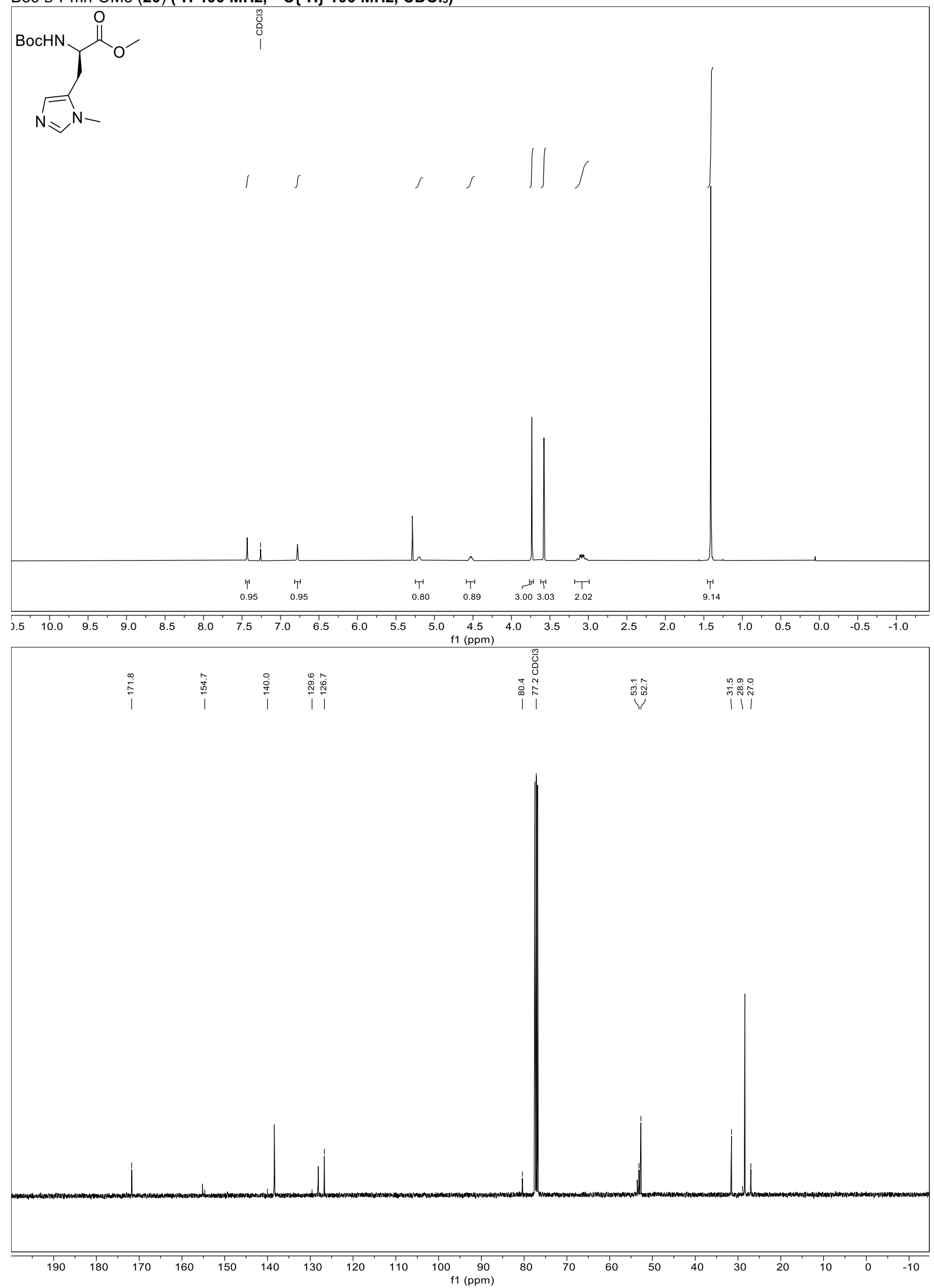



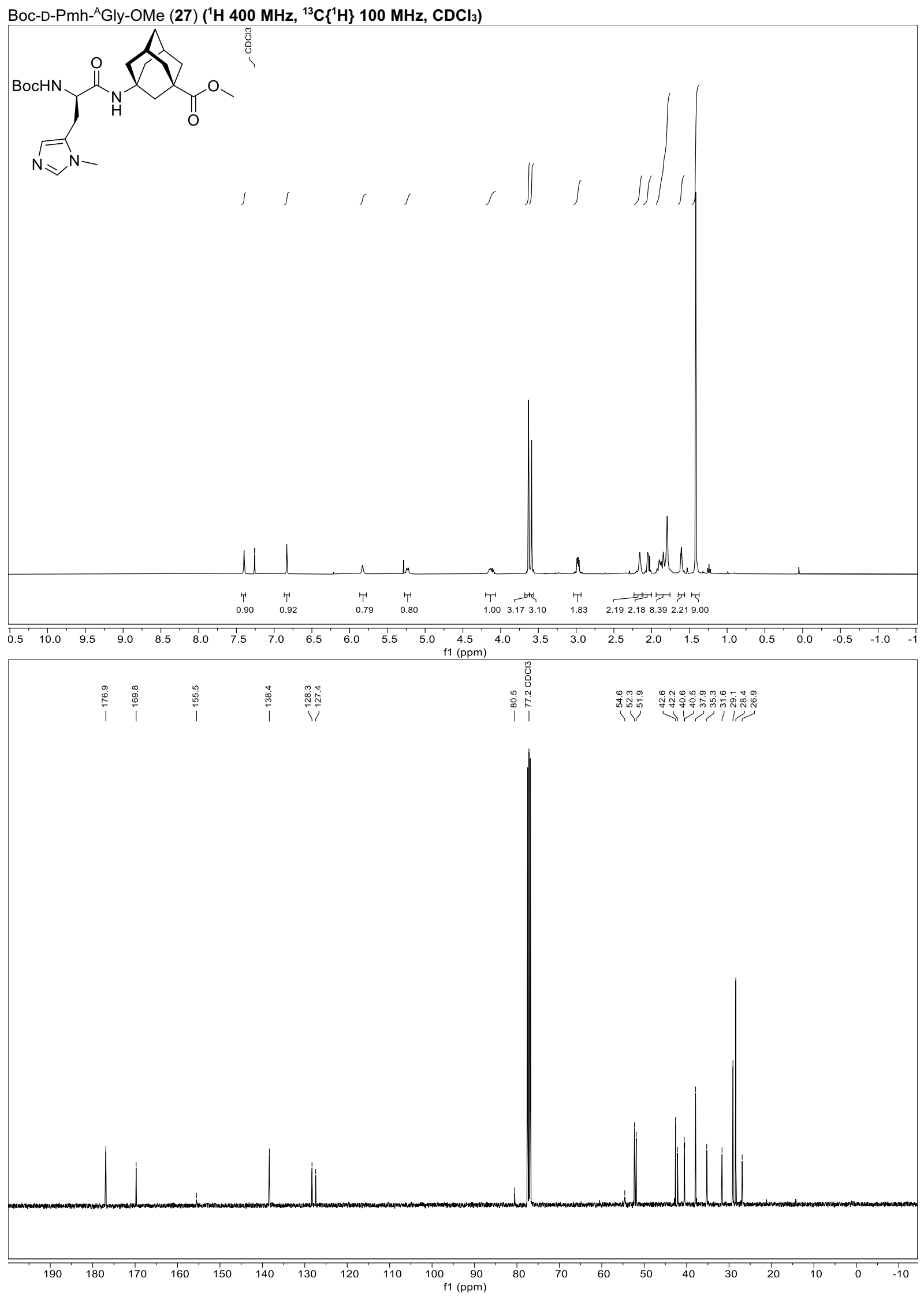

S74 
Boc-L-Pmh- ${ }^{\mathrm{A}} \mathrm{Gly}-\mathrm{L}-\mathrm{Val}-\mathrm{OMe}(28)\left({ }^{1} \mathrm{H} 400 \mathrm{MHz},{ }^{13} \mathrm{C}\left\{{ }^{1} \mathrm{H}\right\} 100 \mathrm{MHz}, \mathrm{CDCl}_{3}\right)$
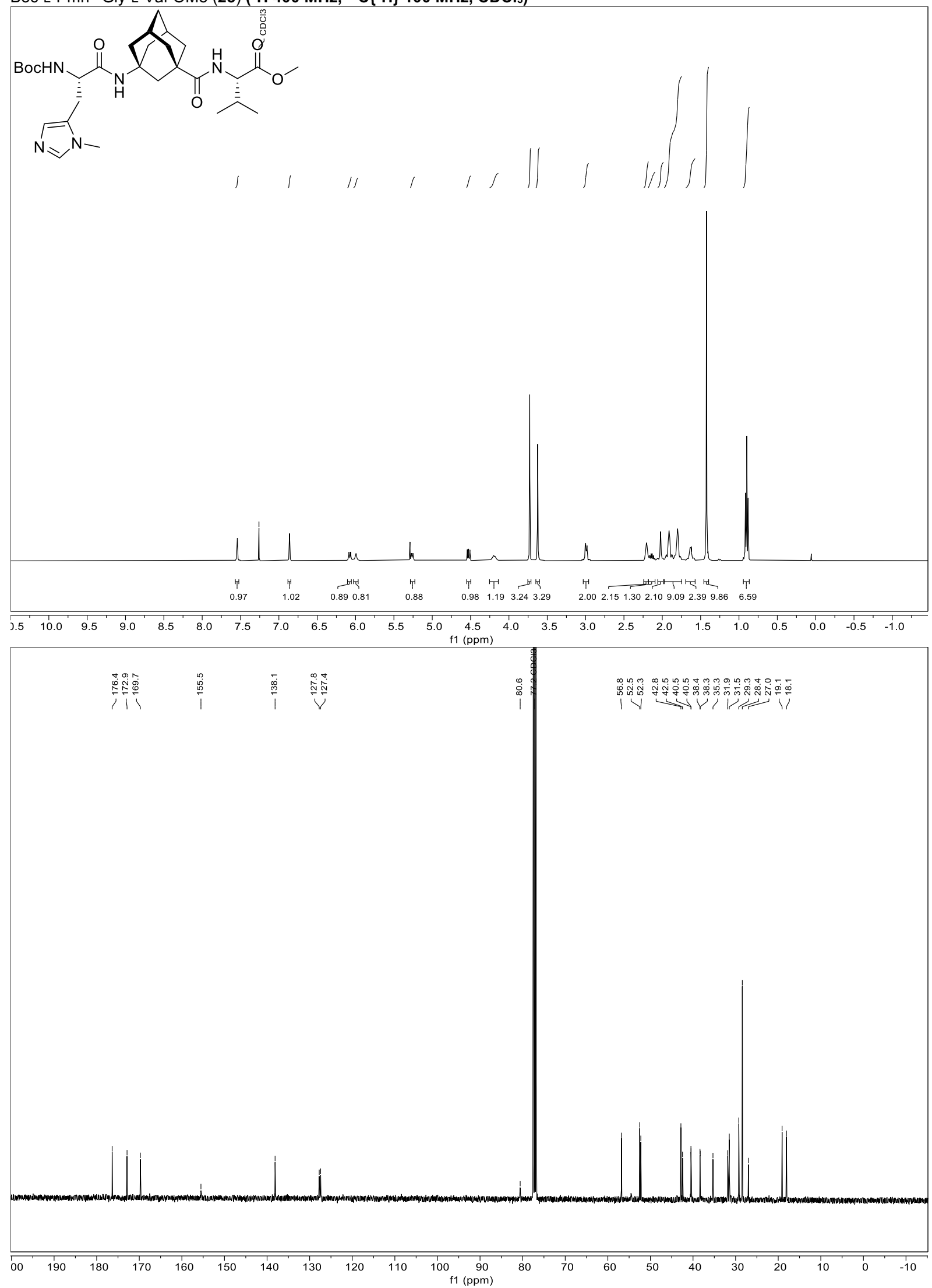
Boc-D-Pmh- ${ }^{\mathrm{A}}$ Gly-L-Val-OMe (29) $\left({ }^{1} \mathrm{H} 400 \mathrm{MHz},{ }^{13} \mathrm{C}\left\{{ }^{1} \mathrm{H}\right\} 100 \mathrm{MHz}, \mathrm{CDCl}_{3}\right)$
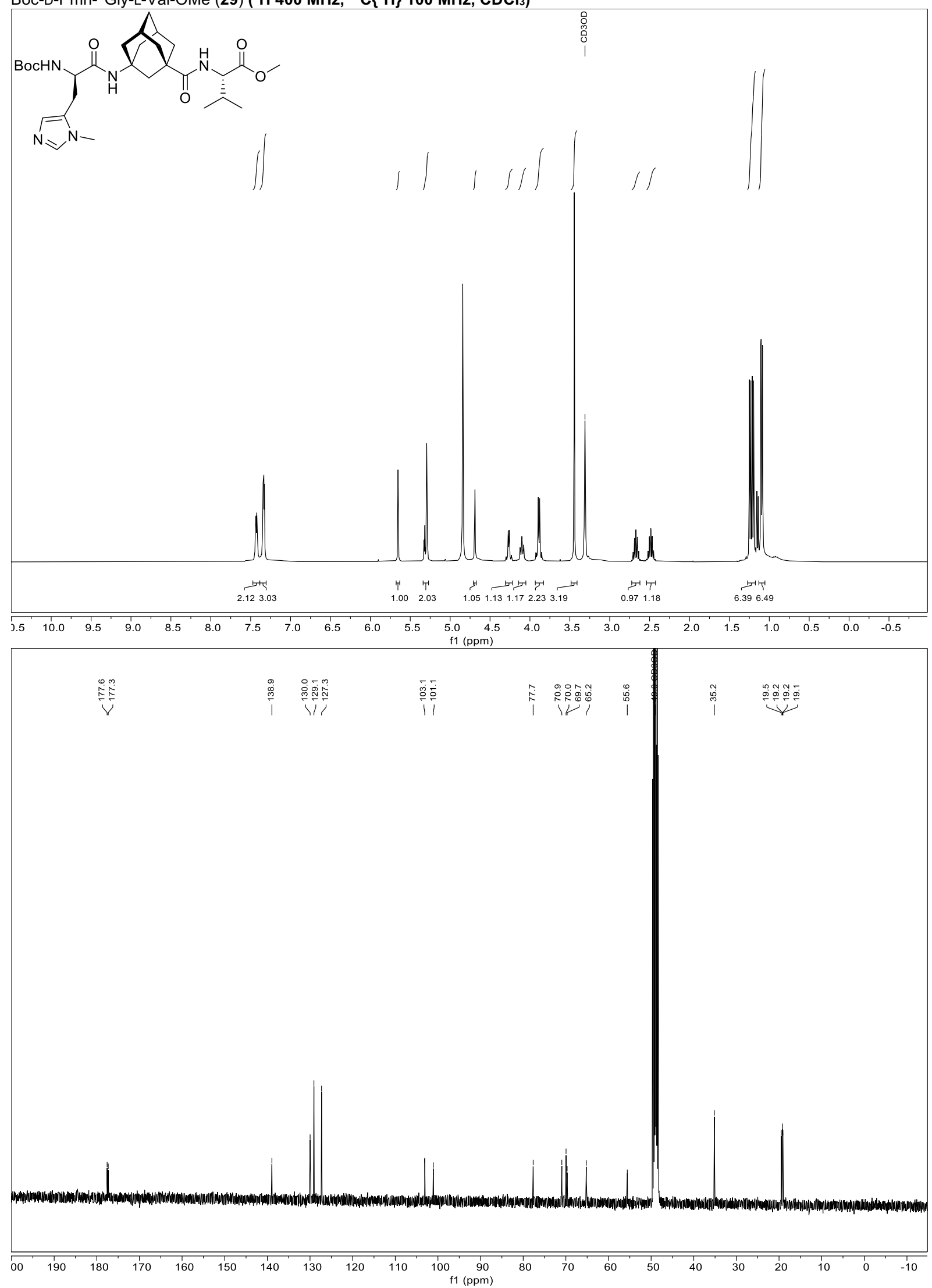
Boc-D-Pmh- ${ }^{\mathrm{A}}$ Gly-L-Cha-L-Phe-OMe (31) $\left({ }^{1} \mathrm{H} 400 \mathrm{MHz},{ }^{13} \mathrm{C}\left\{{ }^{1} \mathrm{H}\right\} 100 \mathrm{MHz}, \mathrm{CDCl}_{3}\right)$

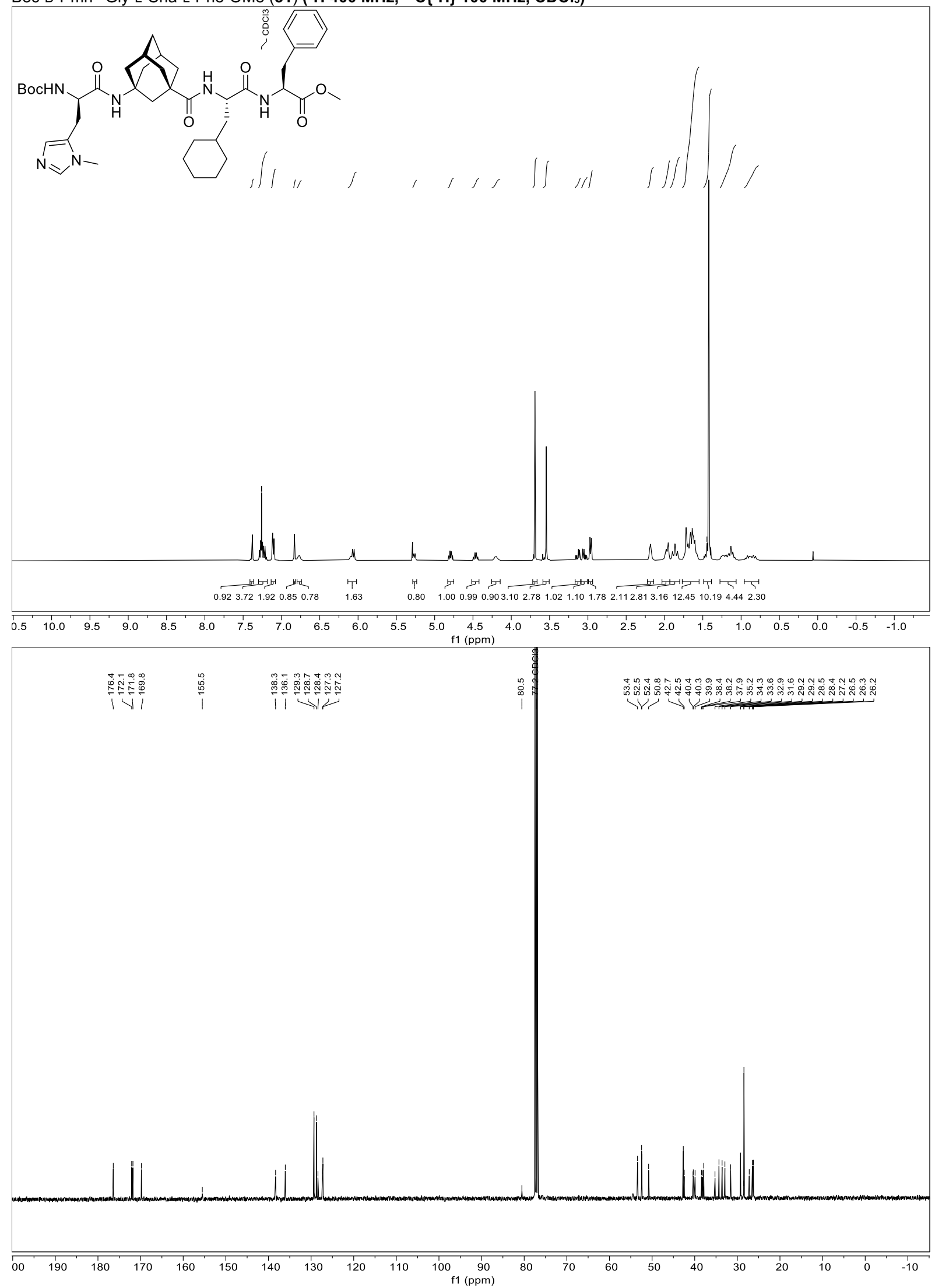




\section{References}

1. $\quad J M P^{\circ}$, Version 14.0.0; SAS Institute Inc.: Cary, NC, 1989-2019.

2. Niedek, D.; Erb, F. R.; Topp, C.; Seitz, A.; Wende, R. C.; Eckhardt, A. K.; Kind, J.; Herold, D.; Thiele, C. M.; Schreiner, P. R., In Situ Switching of Site-Selectivity with Light in the Acetylation of Sugars with Azopeptide Catalysts. J. Org. Chem. 2020, 85 (4), 1835-1846.

3. Ren, B.; Zhang, M.; Xu, S.; Gan, L.; Zhang, L.; Tang, L., DBN-Catalyzed Regioselective Acylation of Carbohydrates and Diols in Ethyl Acetate. Eur. J. Org. Chem. 2019, 2019 (29), 4757-4762.

4. Ren, B.; Gan, L.; Zhang, L.; Yan, N.; Dong, H., Diisopropylethylamine-triggered, highly efficient, self-catalyzed regioselective acylation of carbohydrates and diols. Org. Biomol. Chem. 2018, 16 (31), 5591-5597.

5. Tong, M. L.; Huber, F.; Taghuo Kaptouom, E. S.; Cellnik, T.; Kirsch, S. F., Enhanced siteselectivity in acylation reactions with substrate-optimized catalysts on solid supports. Chem. Commun. 2017, 53 (21), 3086-3089.

6. Allen, C. L.; Miller, S. J., Chiral Copper(II) Complex-Catalyzed Reactions of Partially Protected Carbohydrates. Org. Lett. 2013, 15 (24), 6178-6181.

7. Zhou, Y.; Rahm, M.; Wu, B.; Zhang, X.; Ren, B.; Dong, H., H-Bonding activation in highly regioselective acetylation of diols. J. Org. Chem. 2013, 78 (22), 11618-11622.

8. Eby, R.; Webster, K. T.; Schuerch, C., Regioselective alkylation and acylation of carbohydrates engaged in metal complexes. Carbohydr. Res. 1984, 129, 111-120.

9. Hanessian, S.; Kagotani, M., Novel methods for the preparation of partially acetylated carbohydrates. Carbohydr. Res. 1990, 202, 67-79.

10. Lu, X.-A.; Chou, C.-H.; Wang, C.-C.; Hung, S.-C., Regioselective Esterification of Various dGlucopyranosides: Synthesis of a Fully Protected Disaccharide Unit of Hyaluronic Acid. Synlett 2003, 2003 (09), 1364-1366.

11. Cipolla, L.; Lotti, M.; De Gioia, L.; Nicotra, F., Application of Site-Directed Lipase Mutants on Regioselective Acylation of Monosaccharides. J. Carbohydr. Chem. 2003, 22 (7-8), 631-644.

12. Panza, L.; Luisetti, M.; Crociati, E.; Riva, S., Selective Acylation of 4,6-O-Benzylidene Glycopyranosides by Enzymatic Catalysis. J. Carbohydr. Chem. 1993, 12 (1), 125-130. 